\title{
Context dependent face selection in a visual and semantic perspective
}

Citation for published version (APA):

Gentile, F. (2011). Context dependent face selection in a visual and semantic perspective. [Doctoral Thesis, Maastricht University]. Datawyse / Universitaire Pers Maastricht. https://doi.org/10.26481/dis.20110225fg

Document status and date:

Published: 01/01/2011

DOI:

10.26481/dis.20110225fg

Document Version:

Publisher's PDF, also known as Version of record

\section{Please check the document version of this publication:}

- A submitted manuscript is the version of the article upon submission and before peer-review. There can be important differences between the submitted version and the official published version of record.

People interested in the research are advised to contact the author for the final version of the publication, or visit the DOI to the publisher's website.

- The final author version and the galley proof are versions of the publication after peer review.

- The final published version features the final layout of the paper including the volume, issue and page numbers.

Link to publication

\footnotetext{
General rights rights.

- You may freely distribute the URL identifying the publication in the public portal. please follow below link for the End User Agreement:

www.umlib.nl/taverne-license

Take down policy

If you believe that this document breaches copyright please contact us at:

repository@maastrichtuniversity.nl

providing details and we will investigate your claim.
}

Copyright and moral rights for the publications made accessible in the public portal are retained by the authors and/or other copyright owners and it is a condition of accessing publications that users recognise and abide by the legal requirements associated with these

- Users may download and print one copy of any publication from the public portal for the purpose of private study or research.

- You may not further distribute the material or use it for any profit-making activity or commercial gain

If the publication is distributed under the terms of Article $25 \mathrm{fa}$ of the Dutch Copyright Act, indicated by the "Taverne" license above, 
Context dependent face selection in a visual and semantic perspective 
ISBN 9789461590053

C Copyright Francesco Gentile, Maastricht 2011

Cover illustration: taken from the movie "The face of another" (1966)

Cover design: Nick Kilian-Hütten and Francesco Gentile

Production by Datawyse bv / Universitaire Pers Maastricht 
Context dependent face selection in a visual and semantic perspective

\section{Proefschrift}

ter verkrijging van de graad van doctor aan de Universiteit Maastricht op gezag van de Rector Magnificus, Prof. mr. G.P.M.F. Mols volgens het besluit van het College van Decanen,

in het openbaar te verdedigen

op vrijdag 25 februari 2011 om 12.00 uur

door

\section{Francesco Gentile}

Geboren op 14 September 1978 te Rome

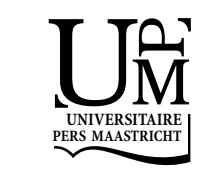




\section{Promotor}

Prof. dr. B.M. Jansma

\section{Copromotor}

Dr. E. Formisano

\section{Beoordelingscommissie}

Prof. dr. L. Blomert (voorzitter)

Dr. V. Goffaux (Université du Luxembourg, Luxembourg)

Prof. dr. T Münte (Universitätsklinikum Schleswig-Holstein, Deutschland)

Dr. B. Rossion (Université catholique de Louvain, Belgique)

Prof. dr. Peter de Weerd 
1. General introduction 1

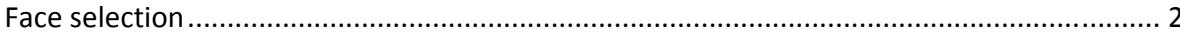

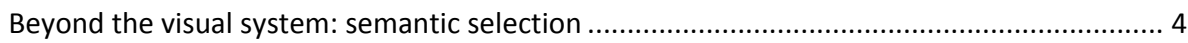

Functional magnetic resonance imaging (fMRI) .................................................................... 5

Electroencephalography (EEG) and Independent Component Analysis (ICA) ........................ 5

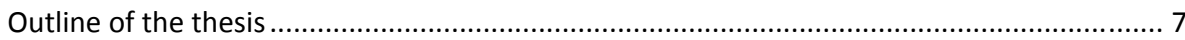

2. Neural competition through visual similarity in face selection 9

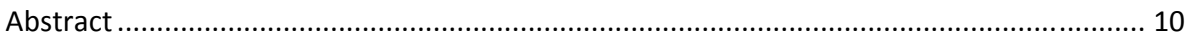

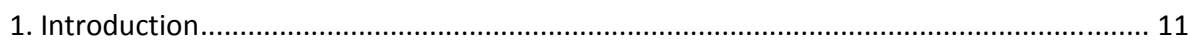

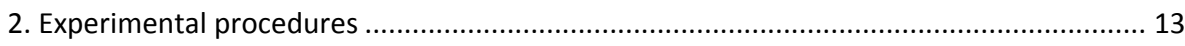

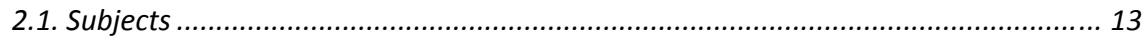

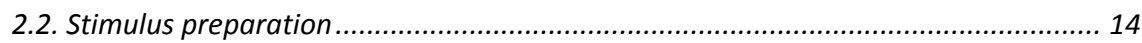

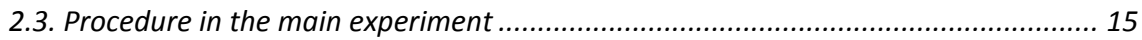

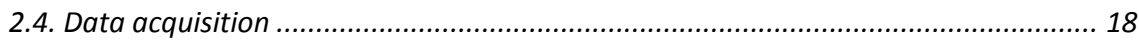

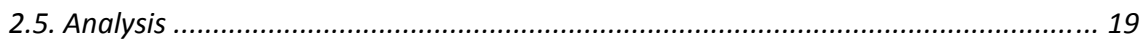

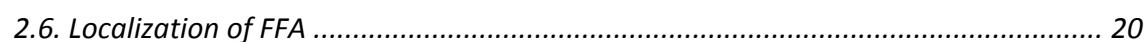

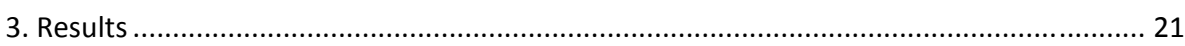

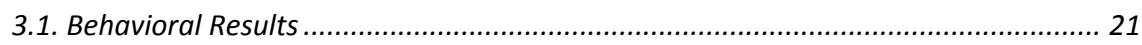

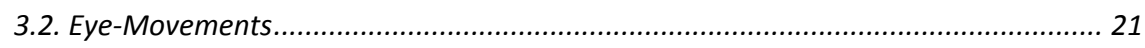

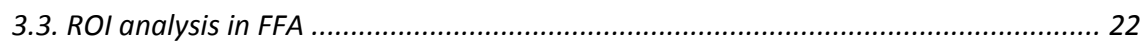

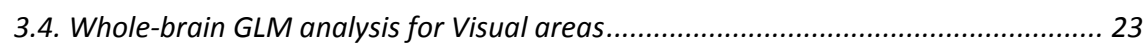

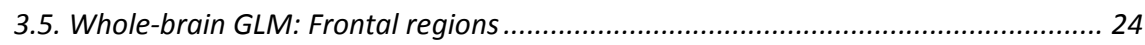

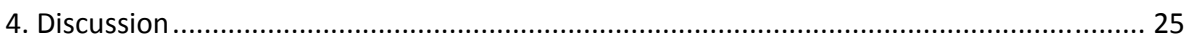

4.1. Neural competition and similarity modulation in FFA and OFA ........................... 26

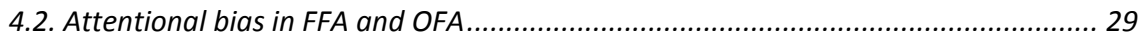

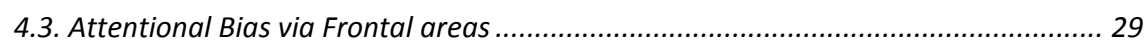

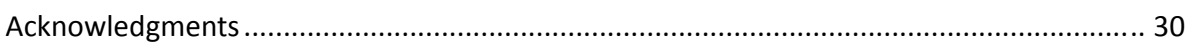

3. Temporal dynamics of face selection mechanism in the context of face processing $\quad 31$

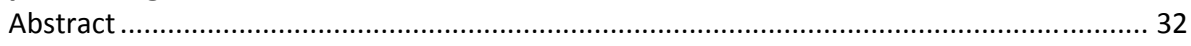

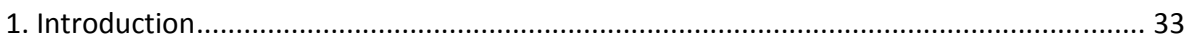

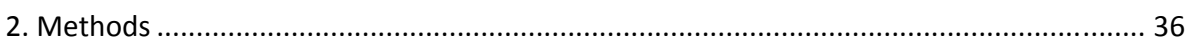

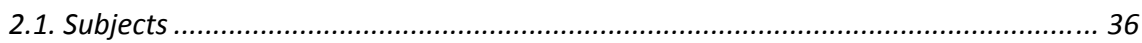




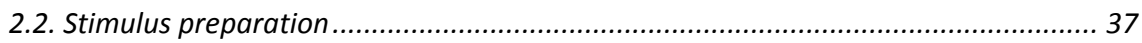

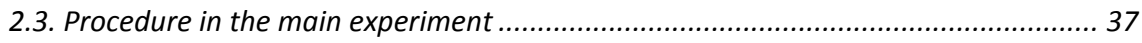

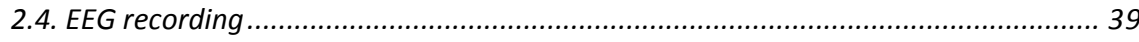

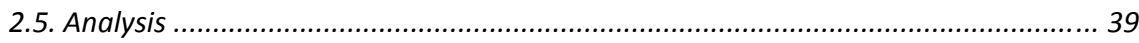

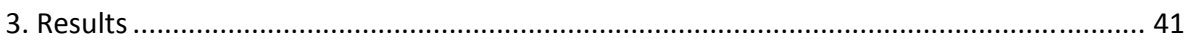

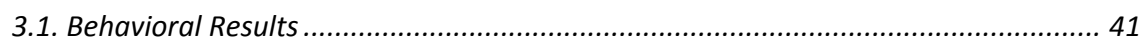

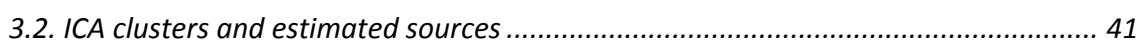

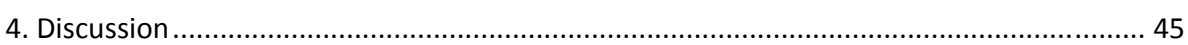

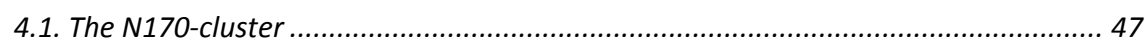

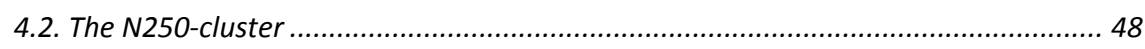

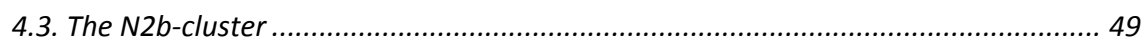

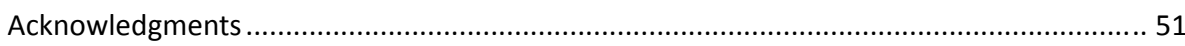

4. Semantic selection in the context of face processing: an fMRI study 53

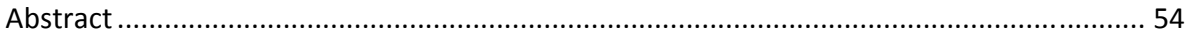

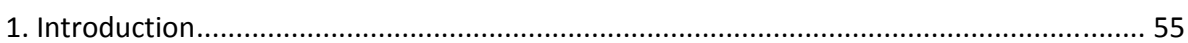

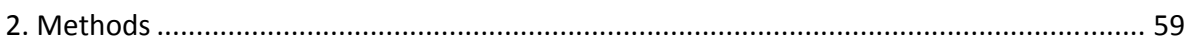

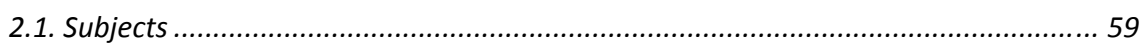

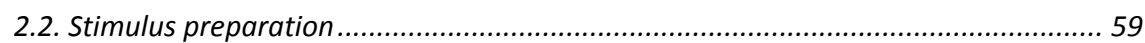

2.3. Procedure in face-profession association phase........................................................ 60

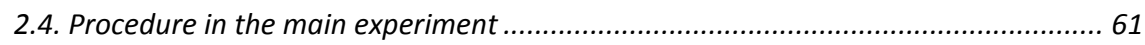

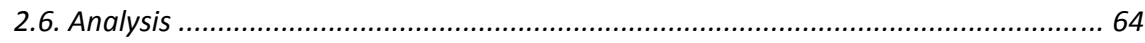

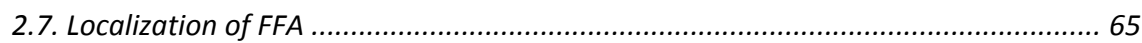

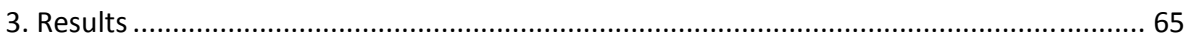

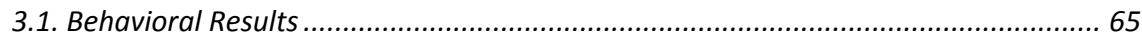

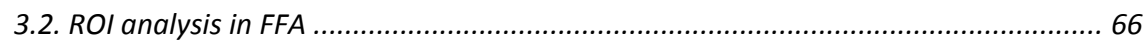

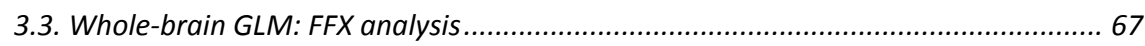

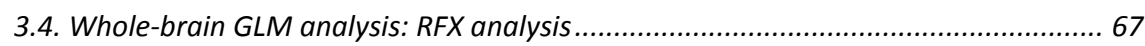

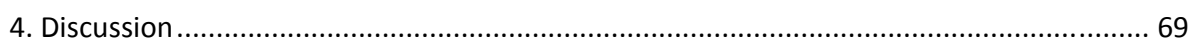

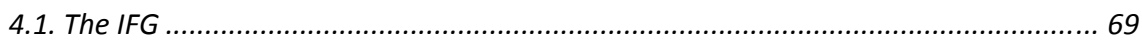

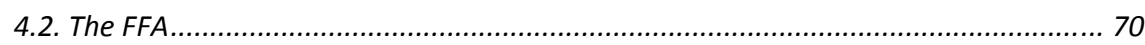

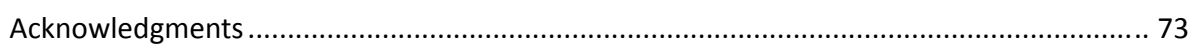

5. Semantic selection in the context of face processing: an EEG study $\mathbf{7 5}$

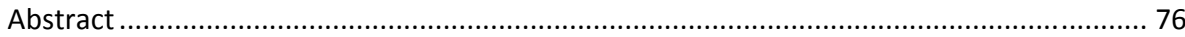

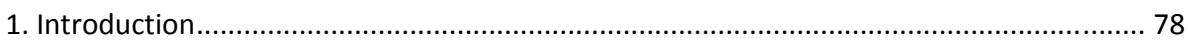

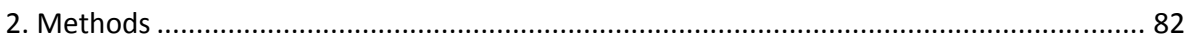

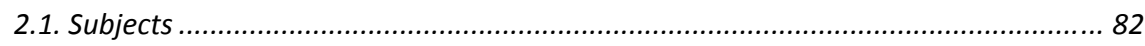

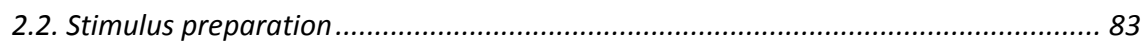

2.3. Procedure in face-profession association phase ..................................................... 83

2.4. Procedure in the main experiment .......................................................................... 83

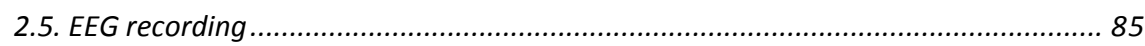

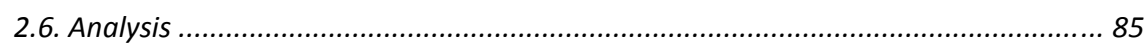

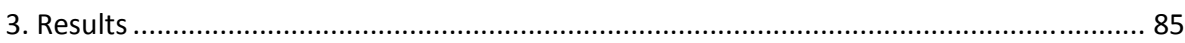

$\mathrm{vi}$ 


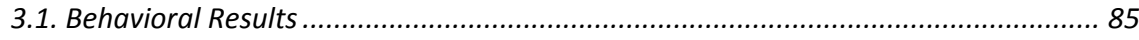

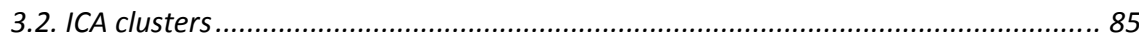

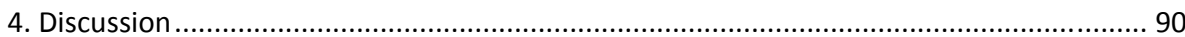

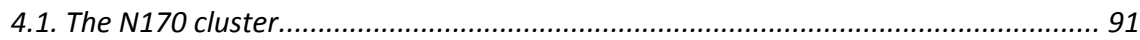

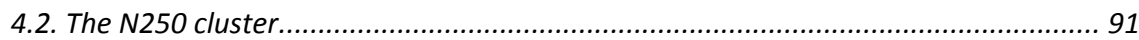

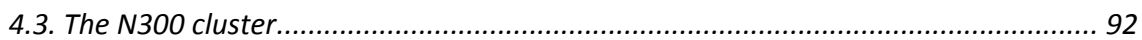

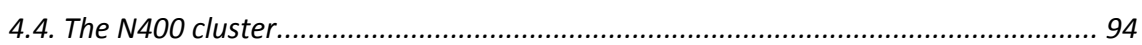

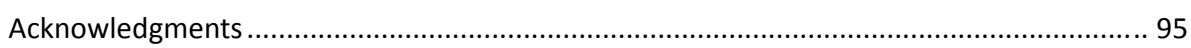

6. General discussion $\quad 97$

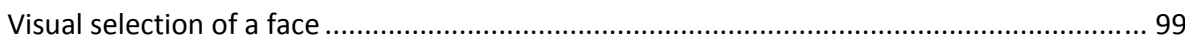

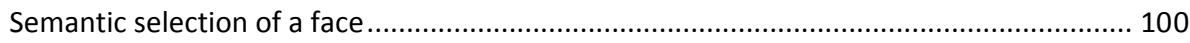

$\begin{array}{ll}\text { References } & 103\end{array}$

$\begin{array}{ll}\text { Summary } & 111\end{array}$

$\begin{array}{ll}\text { Samenvatting } & 115\end{array}$

$\begin{array}{lr}\text { Acknowledgments } & 119\end{array}$

$\begin{array}{lc}\text { Curriculum Vitae } & 123\end{array}$ 

General introduction 
As social beings, the interaction with our peers is necessary in many situations in everyday life. Communication constantly evolved mainly with regard of the way we transfer our knowledge or experience to another person. Nowadays technology provided us with several means to get into contact with friends (e.g. via e-mail, phone or computer). Despite that, the direct face-to-face conversation still remains the most effective way to communicate. When we have an appointment with someone in a public place, we search for her (or him) based on what we know about this person. Our search and the subsequent conversation are guided by visual and semantic background information we retrieve from our memory. So far, it is still unclear how both these types of information are associated to a person. At the cognitive level this integrative process can be divided in two main stages: the access of the visual and semantic face information and its selection. In particular, information access is automatic and takes place also in the absence of awareness. In contrast, the selection of the relevant information occurs via attentional mechanisms.

In order to mimic a typical social situation, we presented the target face (our friend) in the context of another face (surrounding people). The access stage would consist of the preselective encoding of the information extracted from both faces. At the neural level, this phase of visual processing of multiple items is frequently referred to as competition, as several alternative candidates (visual and/or semantic features) compete with each other in order to be represented. These competitive interactions are resolved only when the possible options are reduced to one candidate or unambiguous representation. This is accomplished by attending to one single face at the time which also allows to further process the selected face at a higher level of cognition. This would, for example, correspond to the detection of a friend in a crowd or the recalling of the profession of a person we did not see for long time. This thesis aims to investigate how our cognitive and neural system process visual and semantic information when multiple candidates are accessed at the same time, how these information are integrated and the mechanisms responsible to the selection of the information relevant for behavior.

\section{Face selection}

In several circumstances, we are often surrounded by several people. Our system is not able to process all of them at a given moment. For example if we have to find a friend in a crowd we need to look at every single face in order to accomplish the task. In case we have to search for someone we barely know, additional care is needed to analyze the visual features of the faces encountered and match them with the one corresponding to the target. The efficiency of this matching process can be enhanced by filtering out the irrelevant faces and their associated information. This would, in fact, minimize their interference with target processing. 
Attentional mechanisms are needed to properly perform this task, which is also refereed to as a visual selection task. The behavioral and neural effect of attention has been studied for decades. More specifically, attention to a particularly salient item (bottom-up attention) or to an object relevant for behavior (top-down attention) improves the processing of the stimulus attended to (Broadbent D, 1958; Carrasco M et al., 2004; Desimone R and J Duncan, 1995; Hawkins HL et al., 1990; Luck SJ et al., 1994; Treisman AM, 1969). Several attentional models accounted for this effect. According to the classical view attention works as a spotlight which enhances the neural response of the object placed at the target location or characterized by the relevant feature (Brefczynski JA and EA DeYoe, 1999; Hillyard SA and TF Munte, 1984; Luck SJ and SA Hillyard, 1994; Posner MI et al., 1980; Reynolds JH et al., 2000). Next to the effect on feature detection of stimuli presented in isolation, attention was also widely explored when a single item had to be selected in a cluster of other objects. The most influential model in this context is the Biased Competition (BC) model. This model states that when several items are simultaneously presented they interact with each other for representation. Attention to one of them inhibits the influence of the distractor and competition is fully resolved in favor of the relevant object. Therefore, attention is not considered anymore as a spotlight which enhances the neural response of the stimulus attended to but rather as a bias towards the neural processing of the target. This model was initially tested in single cell recording in primates (Desimone R, 1998; Reynolds JH and R Desimone, 1999) and it, recently, was supported by several fMRI studies in humans (Beck DM and S Kastner, 2005, 2007; Bles M et al., 2006; Kastner S et al., 1998; Kastner S et al., 2001; Kastner S et al., 1999).

In the present thesis we investigated the $\mathrm{BC}$ model for face processing. In particular, we tested whether in absence of any selection two simultaneously presented faces interact in a competitive manner. For this purpose we designed an ignore-faces task where participants were asked to ignore both faces. We also explored the selection phase of the model via an attend-to-face condition where participants attended to one of the two faces and performed a match-to-sample task. In line with the BC model we expected that, in this case, competition would be biased in favor of the visual processing of the target face. In order to test the occurrence of competitive interactions and the bias effect, both tasks were combined with the manipulation of the context. The context was represented by the second face which could be either visually similar or dissimilar to the face of interest. We expected that the simultaneous processing of faces in the ignore-face task would result in a context-dependent modulation of neural activity. In particular, the processing of two similar faces would lead to a stronger competitive effect due to the higher overlap in neural representation of their visual information. In contrast, we did not expect the same context effect in the attend-toface condition. In this case, attentional bias was supposed to resolve the influence of the percept associated to the second face by processing the target only. 


\section{Beyond the visual system: semantic selection}

Let's now imagine that we finally found our friend or acquaintance and we engaged in a conversation with him/her. The fate of a successful interaction depends on the background knowledge we are able to retrieve about that person. In this case, the cognitive system has to accurately associate specific semantic information, stored in our memory, with the target face which in turn is processed at the semantic level. Moreover, if the target is embedded in a cluster of other faces, attention is needed to reduce the influence of these faces and their associated semantic information. The integration between the semantic and visual feature of a face and the filtering of irrelevant information are the core processes of the semantic processing associated to a face.

In psycholinguistic research, both in language comprehension and production, the semantic access of a visual stimulus (i.e., the analysis of all the possible semantic entries associated to it) and the selection of an appropriate semantic representation are studied by means of various paradigms. In particular, in production, the most common paradigm is the picture naming task. A typical design in this context consisted of the presentation of the picture of a specific object that was associated either with just one or with different name alternatives. Participants were asked to select one semantic representation among all the possible options. It has been shown that naming latencies and errors increased with the increasing amount of alternative semantic entries associated to the picture (Kan IP and SL Thompson-Schill, 2004; Kremin H et al., 2000; Levelt WJM et al., 1991; Thompson-Schill SL et al., 1997; Vigliocco $\mathrm{G}$ et al., 2002). According to the authors this result suggested that several semantic representations compete with each other. In analogy to the competitive model of selection in the visual domain, semantic selection is accomplished by resolving these competitive interactions in favor of the relevant semantic information. However, this model was never directly investigated. In fact, in the reported tasks designed to test semantic selection, both competition and selective processes were engaged at the same time.

This thesis aimed to explore these two mechanisms separately in the context of face processing. We presented two faces that were semantically related or unrelated (physical similarity was kept constant). The semantic relatedness manipulation was obtained by associating each face in a pair with professions belonging to the same (related faces) or different (unrelated faces) categories. The simultaneous encoding and the competitive interactions between the two semantic representations of faces were tested by an ignore-faces task, similar to the one used in the experiments on visual selection. We expected that semantic related areas would process the semantic information from both faces. This in turn would cause the two faces to interact in a competitive manner at the semantic level, leading to a modulation by the context (relatedness). Selection of the semantic information associated with the target face was explored by an attend-to-face task. Also in this case, as in the visual domain, participants had to attend to one of the faces. But instead of selecting the face according to specific visual characteristics, they were asked to decide whether a face was 
associated with a specific profession (match-to-sample task). We expected that attention to the relevant face would resolve the competition effect occurring in the ignore-faces condition. With the attend-to-face condition we also tested whether and how the initial perceptual analysis of the target face interacted with the semantic selection process.

\section{Functional magnetic resonance imaging (fMRI)}

fMRI is an indirect and non-invasive measure of the neural response related to sensory stimulation or cognitive processes. The fMRI signal reflects the metabolic activity of neurons which is assumed to be positively correlated with their neural activity. In fMRI, the subjects undergo a strong constant magnetic field (e.g. 3 Tesla) which aligns the protons in the hydrogen atoms. A radiowave signal is sent out in order to make the protons oscillate. The fMRI detector measures the local energy fields emitted when protons return to equilibrium (the orientation of the external magnetic field). In particular, when a certain area of the brain is active, neurons increase the oxygen consumption. However, the system overcompensates for this oxygen demand such that an excess of oxygenated hemoglobin is delivered to the activated region. This surplus causes in the same brain tissue a reduction of the amount of deoxygenated hemoglobin. Moreover, as the deoxygenated hemoglobin is paramagnetic, it produces magnetic inhomogeneities in the capillary which in turn increases the rapidity of the MR signal decays. Therefore, a smaller amount of deoxygenated hemoglobin during neural activity results in a slower decay of the MR signal and thereby in an enhancement of the signal compared to a non active region (baseline) (DeYoe EA et al., 1994).

It is worthwhile to notice that the dynamic of processes described above is very slow, typically on the range of several seconds which makes the temporal resolution of fMRI relatively low. In contrast, this technique provides an excellent spatial resolution. In fact the fMRI is able to detect changes in the ratio of oxygenated and deoxygenated hemoglobin in a range of few millimeters or less.

\section{Electroencephalography (EEG) and Independent Component Analysis (ICA)}

When a sensory stimulus is presented or a cognitive process takes place, we can identify mainly two types of electrical activity associated with neurons: the action potentials and the postsynaptic potentials. The EEG signal is measured by superficial electrodes placed on the scalp of the tested subject and reflects the postsynaptic potentials. This type of electrical activity originates when the action potential reaches the axon terminal and the neurotransmitters are released and bind to receptors located on the membrane of the postsynaptic neuron. This, in turn, causes the ion channels to open which allows electrical charges like sodium and potassium ions to travel through those membrane channels. This mechanism leads to a potential change, also known as postsynaptic potential. Postsynaptic potentials 
occur instantaneously and they can be modeled as tiny dipoles. Superficial electrodes are not able to measure the voltage from a single neuron. The electrical signal detected on the scalp (EEG signal) reflects the dipole activity of hundreds of neurons that are oriented along the same direction and respond to sensory stimuli or process a cognitive task in the same way at the same time. Postsynaptic activity, in such a case, sums up to a measureable electrical potential on the scalp.

In order to extract the neural response, evoked by sensory stimuli or phase locked cognitive processes, the ongoing EEG is averaged across several presentations of the same experimental condition. The averaging of the EEG signal is in fact necessary to amplify the time locked process of interest and to cancel out any random neural activity over time. The resulting time courses are referred to as event-related-potentials (ERPs). ERPs are characterized by the morphology of the signal which is represented by prominent positive $(P)$ and negative $(\mathrm{N})$ peaks at specific latencies with respect to the evoking neural event.

In summary, the EEG technique provides a non invasive measure of neural activity with very high temporal resolution (few milliseconds). However, due to the phenomenon of volume conduction, the electrical signal from the cortex is strongly distorted when it reaches the surface. In addition, the EEG signal at a certain electrode can be originated from a distributed rather than a single source. This problem of locating the brain sources of the recorded data, also known as the 'EEG inverse problem', is mathematically ill-posed and is not resolvable without additional constraints and assumptions. Because it is impossible to retrieve where an observed signal has actually been generated, the EEG is characterized by a rather low spatial resolution (Kutas M and AM Dale, 1997).

We analyzed the data by using the independent component analysis (ICA) (Onton J and $S$ Makeig, 2006). The ICA model assumes that the neural activity generated on the cortex is spatially constant and projects onto the scalp instantaneously and linearly via volume conduction. It also states that these cortical sources are statistically independent of each other. This assumption arises from the observation that the density of local synaptic contacts among pyramidal cells is much larger than the long-range connections. Therefore, across a reasonable amount of time, it is plausible to consider the brain as divided into separate functional areas that contribute to temporally independent neural processes that sum up linearly on the scalp. These brain sources that give rise to the signal recorded over the scalp correspond to the independent components (ICS). In fact, the ICA algorithm takes as input the EEG signal recorded at the scalp channels and considers each of these signals as a linear mixture of temporal independent components. The ICs represent the time courses in the mixture that are maximally independent to each other. 


\section{Outline of the thesis}

The general purpose of the present thesis was to investigate the underlying neural mechanism of visual and semantic selection of a face in the context of another face. In particular we tested the competitive models of selection both when a face had to be selected with respect to its visual features or to specific semantic information. In Chapter 2 and Chapter 3 we tested this model in the visual domain by performing an fMRI and EEG experiment. We exploited the high spatial resolution of $\mathrm{FMRI}$ to investigate the competition and the selection phase within face selective areas. The EEG was used to track the temporal dynamics of both mechanisms. We assumed that in the ignore-faces task the two faces would be encoded and compete for representation at an early stage of face processing. In a later phase we expected that the selection would occur. We predicted that attention would filter out the influence of the distracting face via frontal areas resulting in a context-independent processing of the target face. In Chapter 4 and Chapter 5 we attempted to generalize the competitive models of selection in the semantic domain. The spatial and temporal characteristics of semantic selection were investigated by using fMRI and EEG. In analogy to the visual experiments, the two phases of the model (competition and selection) were explored with an ignore-face and an attend-to-face condition. When the two faces were ignored, we expected the face information to be encoded in semantic related areas and to compete to a certain extent. In the attend-to-face condition, which in this case consisted in a match-to-profession task, we expected that selection would occur in two distinct stages. In an early phase we predicted that the information carried by the distracting face would interfere with the integration of the attended face and the associated semantic information. Attention, in turn, would compensate for this interference effect in order to facilitate the actual selection occurring in a later stage within those regions responsible to semantic selection. 



\section{Neural competition through visual similarity in face selection ${ }^{1}$}

\footnotetext{
${ }^{1}$ Corresponding publication: Gentile F and Jansma BM, Neural competition through visual similarity in face selection, BrainRes (2010), pp. 172-184 (in press)
} 


\begin{abstract}
Ignoring and selecting relevant faces has a strong impact in everyday life. We often perform tasks where faces may be considered irrelevant (e.g. having a drink in a crowded bar) or tasks where we need to filter out all but one face (e.g. searching for a friend in a crowd). The present study was designed to test Biased Competition (BC) as a suitable model for selection in the context of face processing, using functional magnetic resonance imaging (fMRI). Pairs of similar or dissimilar faces were presented simultaneously, and subjects had to attend to one face (target face) or ignore both faces. According to the BC model, faces simultaneously presented compete for representation. Spatial attention biases these competitive interactions towards neural processing of the target face only. We compared fMRI signal related to the processing of dissimilar or similar faces in the attend-to-face and ignore-faces task. In the ignore condition we expected that similar faces would compete more than dissimilar faces, as similar features (faces) are supposed to be encoded by the same population of neurons, resulting in a lower $\mathrm{FMRI}$ signal in face selective areas. The BC model also predicts an enhancement of the fMRI signal for the attend-to-face vs. ignore-faces condition, regardless of the degree of the similarity between the two faces. Both hypotheses were confirmed by the data, indicating $B C$ as a possible selection mechanism within the fusiform face area (FFA) and occipital face area (OFA) for face stimuli.
\end{abstract}

Keywords: face perception, neural competition, attention, visual similarity, face selective areas, fMRI 


\section{Introduction}

The visual system receives many stimuli at the same time. Attentional mechanisms are needed to guide visual processing for relevance (Broadbent D, 1958; Desimone R and J Duncan, 1995; Treisman AM, 1969). It is widely assumed that attention improves the processing of behaviorally relevant objects (Carrasco M et al., 2004; Hawkins HL et al., 1990; Luck SJ et al., 1994). According to the influential spotlight metaphor, attention enhances the neural response for stimuli presented at the relevant location (Brefczynski JA and EA DeYoe, 1999; Hillyard SA and TF Munte, 1984; Luck SJ et al., 1994; Posner MI et al., 1980; Reynolds JH et al., 2000).

Next to its relevance in optimizing visual processing (facilitation), spatial attention also plays a crucial role in segregating one item from a cluster of irrelevant objects (selection). Monkey cell recording studies investigated the effect of selective attention in the ventral and dorsal visual stream. In these experiments, a pair of items was presented at the same time within the receptive field of the target neurons. When stimuli were ignored, the neural response to both items was a weighted average of the response elicited by each of the stimuli alone. However, when attention was shifted to one of the two objects, the response to the pair was driven preferentially by the attended stimulus (Chelazzi L et al., 1998; Chelazzi L et al., 2001; Desimone R and J Duncan, 1995; Luck SJ et al., 1997; Miller EK et al., 1993; Moran J and R Desimone, 1985; Reynolds JH et al., 1999; Treue S and JC Martinez Trujillo, 1999; Treue S and JH Maunsell, 1996). These findings gave rise to models for attentional selection based on competitive interactions between neural representations of stimuli. One of the most influential competitive models is Biased Competition (BC). The BC model states that multiple stimuli that are simultaneously presented to the receptive field of neurons under investigation compete for neural representation (Desimone R, 1998; Desimone R and J Duncan, 1995; Kastner S and LG Ungerleider, 2000, 2001; Pessoa L et al., 2003; Reynolds JH and $\mathrm{R}$ Desimone, 1999). Local and intracortical connections seem to regulate this mechanism of neural competition, usually observed for ignored stimuli (Reynolds JH and R Desimone, 1999). Attending to a stimulus activates a fronto-parietal network, which biases the competition in favor of the attended item (target), via feedback towards perceptual areas. As a result, neurons that respond to the target stimulus remain active, while neurons that respond to the ignored stimuli are suppressed.

Further evidence in favor of BC in early visual processing has come from human functional magnetic resonance imaging ( $\mathrm{fMRI}$ ) studies using abstract visual objects. $\mathrm{BC}$ has been mainly tested by presenting stimuli either sequentially (no competition) or simultaneously (competition) (Beck DM and S Kastner, 2005, 2007; Kastner S et al., 1998; Kastner S et al., 2001; Kastner S et al., 1999). In these studies, the fMRI signal was significantly lower for simultaneously presented compared to sequentially presented objects. The authors inter- 
preted this effect as neural suppression, supporting the idea that stimuli compete for representation in the visual cortex. In subsequent experiments, the role of attention within the BC framework was also investigated. In line with electrophysiological studies in monkeys, human attentional mechanisms also seem to influence neural competition. In particular, the fMRI signal increased either when one stimulus was particularly salient (Beck DM and $S$ Kastner, 2005) or when one of the items was covertly attended to (Bles M et al., 2006; Kastner $\mathrm{S}$ et al., 1999). This enhancement suggested that attention can bias the neural competition and thereby facilitate the selection of the attended item. Note that all these studies used rather abstract stimuli such as Gabor patches, or colorful pattern pictures, and had a focus on early visual processing. In the present study, we tested the BC model in the context of face processing.

Face processing has been largely investigated in humans using electroencephalography (EEG) and fMRI. In particular, event-related potential (ERP) studies showed a significant difference for a negative potential around $170 \mathrm{~ms}$ after stimulus onset (N170) when the presented faces were compared to non-face stimuli. This effect was particularly evident at the lateral occipital electrode sites (Bentin S, Allison, T., Puce, A., Perez, E., and McCarthy, G., 1996; Rossion B et al., 1999). In the last decade, fMRI allowed to localize the source of face processing within a region in the fusiform gyrus, which has become known as the fusiform face area (FFA) (Gauthier I et al., 2000; Grill-Spector K et al., 2004; Halgren E et al., 1999; Haxby JV et al., 2001; Kanwisher $\mathrm{N}$ et al., 1997). Furthermore, a combined EEG and magnetoencephalographic study localized the source of the N170 in the fusiform gyrus (Deffke I et al., 2007). However, thus far, the neural mechanism underlying selection of one face among several faces within FFA is still unknown.

To our knowledge, only a few studies examined the neural correlates of processing multiple face displays. Jacques and Rossion (2004, 2006), recently found that the N170 amplitude was smaller when a center target face was presented in a context of other faces than when it was shown with scrambled faces. This effect, referred to as repetition facilitation (or priming), was related to residual activation of neural representation of the preceding face context in the sequence of trials. Alternatively, the observed effect could be related to sensory suppression, which might result from competitive interactions between the items simultaneously presented.

In the present study, we investigated the mechanism of face selection by means of a biased competition paradigm using fMRI. The high spatial resolution of this technique allowed us to restrict the region of interest to FFA and to examine secondary effects in other regions of the brain. The experimental design consisted of two simultaneously presented faces. They had to be either ignored or attended to. With regard to the ignore-faces task, we assumed that simultaneously presented faces are also processed as such in FFA (Halgren E et al., 1999; Reinholz J and S Pollmann, 2007) - hence competing with each other for representation (Desimone, 1998). The attend-to-face task was introduced as a baseline condition for single-face processing (Recanzone GH, 2000; Reynolds JH et al., 1999). We compared the 
neural activation elicited by both tasks to test the competitive aspects of multiple face encoding. In line with the BC model we expected a lower FMRI signal in FFA for the ignore-faces condition compared to the attend-to-face condition.

Neural competition was further investigated by varying the similarity level (similar, dissimilar) of the faces presented. We manipulated similarity in order to draw upon the spatial and functional characteristics of neural populations in FFA. In fact, neural populations in the extrastriate cortex seem to be organized according to the similarity level of the object representations (Op de Beeck HP et al., 2008; Tanaka K, 2003). This hypothesis was recently supported by two fMRI studies (Jiang $X$ et al., 2006; Loffler $G$ et al., 2005), which reported that in FFA two dissimilar faces are encoded by separate populations of neurons. In contrast, similar faces were encoded by cells that were spatially clustered together (Jiang $X$ et al., 2006). A similar finding was reported for faces sharing the same "identities" (Loffler G et al., 2005). At a cell population level, these results suggested that neural competition may vary with the degree of similarity between faces in a pair. Therefore, we expected that neural competition between two face representations would be greater among elements encoded by the same neural population (similar faces) than among elements activating distinct subpopulations of neurons (dissimilar faces). This would result, in turn, in a lower fMRI signal for similar vs. dissimilar faces within the ignore-faces task. The bias component of the $\mathrm{BC}$ framework was investigated by focusing on the attend-to-face task. According to the $\mathrm{BC}$ model, spatial attention towards a specific item in the visual field is supposed to resolve neural competition in favor of the attended item only. In this case we expected no effect of the similarity manipulation. The present experiment was designed in such a way that any sequential priming effect (or adaptation in fMRI terms) was kept constant between conditions (see Stimulus preparation).

Besides a fine grained investigation of FFA, we were also interested in a more general analysis of the whole brain as other areas may be involved in competition and selection. We expected to find a main effect of attention within clusters in the typical fronto-parietal network, consistently activated in various tasks involving spatially directed attention (Corbetta M et al., 1998; Corbetta M et al., 1993; Gitelman DR et al., 1999; Hopfinger JB et al., 2000; Nobre AC et al., 1997; Thomsen T et al., 2005).

\section{Experimental procedures}

\subsection{Subjects}

Nineteen healthy and paid volunteers (10 females, one left-handed) with normal or corrected to normal visual acuity participated in this study (mean age $=25.3$ years, $S D=1.8$ years). After explanation of the procedures, participants signed an informed consent form. The study was approved by the ethical committee of the faculty. In 16 subjects (out of 19) FFA 
was properly localized. As FFA was the main focus of the present investigation, the remaining participants (3 out of 19) were discarded from further analysis. Due to technical difficulties of the button-box, the behavioral responses were recorded for 15 subjects (in the final set of 16).

\subsection{Stimulus preparation}

The stimuli consisted of two sets of 16 pairs of faces. One set represented similar (SIM) and the other dissimilar (DISS) faces (Figure 1.c, 3.a). The faces in the pairs were selected from a large set of pictures of faces (total of 60 pictures) provided by Mark Steyvers (http://psiexp.ss.uci.edu/research/software.htm). The pictures were all taken under similar lightning with neutral facial expressions (Kayser S, 1985).

Similarity between faces was defined by using the two major approaches adopted for face representation: the purely psychological or top-down approach and the purely computational or bottom-up approach (Figure 1.a, b). Both approaches are based on the multidimensional representation of faces (Steyvers M, \& Busey, T. , 2000). In the psychological approach, the data for computing two-face similarity consisted of similarity ratings of each face with all the other faces. Similarities or dissimilarities in these data were explored using scaling techniques. A Multidimensional Scaling algorithm (MDS) assigned to each face a location in a multidimensional space (Steyvers M, 2002) (Figure 1.a). We first computed the Euclidean distance between a given face (i.e. its Cartesian representation in the multidimensional space) and all the others resulting in a 'mutual distance' value (Figure 1.a, b). Secondly, two faces were considered SIM (or DISS), for this specific approach, if their mutual distance was smaller (or larger) than a specific threshold set according to the overall distribution of the mutual distances. In particular, two faces were labeled as similar if the mutual distance between two faces was smaller than the mean of the mutual distance distribution plus 0.70 times its standard deviation. Similarly, if the mutual distance was larger than the mean of the mutual distance distribution plus one time its standard deviation two faces were labeled as dissimilar.

In the computational approach, a face was represented by a collection of features or dimensions ( $N=30$, e.g. nose length, width of the eye brows etc.) explicitly derived from a 2D image of the face (Figure 1.b). The same two-step procedure as described above, for the psychological approach, was used to define pairs of SIM and DISS faces. However, for the latter set an additional constraint was considered. We took into account the observation that faces are encoded by their direction (facial identity) and distance (distinctiveness) from a prototypical (mean) face (Loffler $\mathrm{G}$ et al., 2005). According to this study, visual stimulation of a particular face elicits a different level of activation in FFA depending on its position in a multidimensional space from the mean face. Therefore, in order to control that both faces in the pair equally activated FFA, two faces were considered DISS only if a) their mutual distance was larger than a specific threshold (see above), and if b) they both differed in the 
same amount from the mean face. The mean face was computed from all the faces used in the experiment.

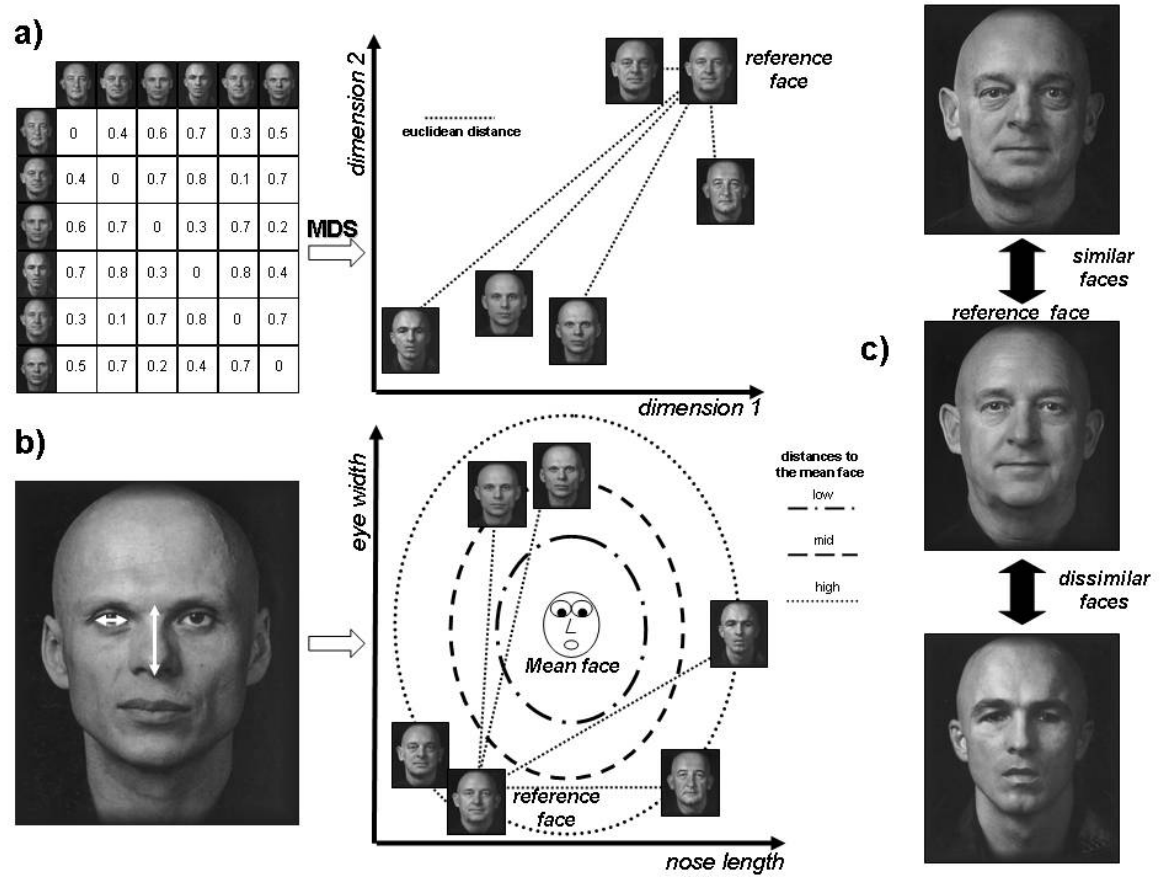

Figure 1. An example of material selection of a similar and dissimilar pair of faces given one face (reference face). (a) Top-down approach of selection. (b) Bottom-up approach of selection. (c) Result of selection: combination of results from both approaches.

The final set of stimuli was derived as follows: a pair of faces was labeled as a SIM (or DISS) pair, only if both faces were labeled as such in the top-down as well as in the bottom-up approach (Figure 1.c). As a last step, we computed the distance of both sets of faces from the mean face in order to avoid differences in the level of activation within FFA, across conditions. SIM and DISS faces were, on average, at the same distance from the mean face.

\subsection{Procedure in the main experiment}

The main experiment consisted of two runs in a block-design (Figure 2). Each run consisted of 32 blocks and started with a blank screen with a white cross in the centre lasting for 18 seconds. A block consisted of 16 trials. A single trial started with a 500 ms of blank screen, followed by a pair of faces presented for $500 \mathrm{~ms}$. The two faces were positioned in opposite hemifields and they were either SIM or DISS to each other (see Stimulus preparation). The single pictures subtended approximately $6.5^{\circ}$ (height) $\times 5.4^{\circ}$ (width) of visual angle and were aligned with the centre of the screen. The entire visual display covered $6.5^{\circ}$ (height) $\times 12.2^{\circ}$ 
(width) of visual angle. The display size was designed to be comparable with those used in previous studies on face processing (Gauthier I et al., 2000; Halgren E et al., 1999; Kanwisher $\mathrm{N}$ et al., 1997; Rossion B et al., 2003; Steeves JK et al., 2006; Wojciulik E et al., 1998). It is also reasonable to assume that FFA integrates the two simultaneously presented faces within each experimental trial (Halgren E et al., 1999; Reinholz J and S Pollmann, 2007). Between the faces, two bars $\left(0.2^{\circ}\right.$ in height) were presented $0.15^{\circ}$ above and below the centre of the visual screen. A block of trials ended with a resting condition consisting of a blank screen lasting for 10 seconds. A visual instruction was presented at the beginning of each block for $1500 \mathrm{~ms}$, and signalled the type of task to be performed in the following block.

For the ignore-faces (IGN) task, two keywords ('streep-breedte', 'line-width' in English) centred on the screen, instructed the subject to perform a bar-width discrimination task in the centre of the display, while ignoring the background (Figure 3.a). More specifically, participants had to attend to the two bars in the centre and a button-press was required when the bars matched in width (2-3 targets per block). The width of the bars varied between 1 and 5 pixels. For the attend-to-face (ATT) task, the instruction consisted of a picture of a face, on which the subject had to perform a match-to-sample task. For each trial, participants were asked to respond via a button press when the face presented on the left side of the screen matched the one presented in the instruction phase (Figure 3.a). For this task, subjects were instructed beforehand to avoid saccades and to covertly attend to the item on the left while fixating the centre. Within each block, targets (matches) occurred on average $15 \%$ of the time (randomly varied between $12.50 \%$ and $18.75 \%$ across blocks and participants). In analogy to preceding biased competition studies (Bles $M$ et al., 2006) targets always occurred at the target position, on the left side of the visual field. In both tasks, the time for the subject to respond was $1000 \mathrm{~ms}$ (trial duration). 


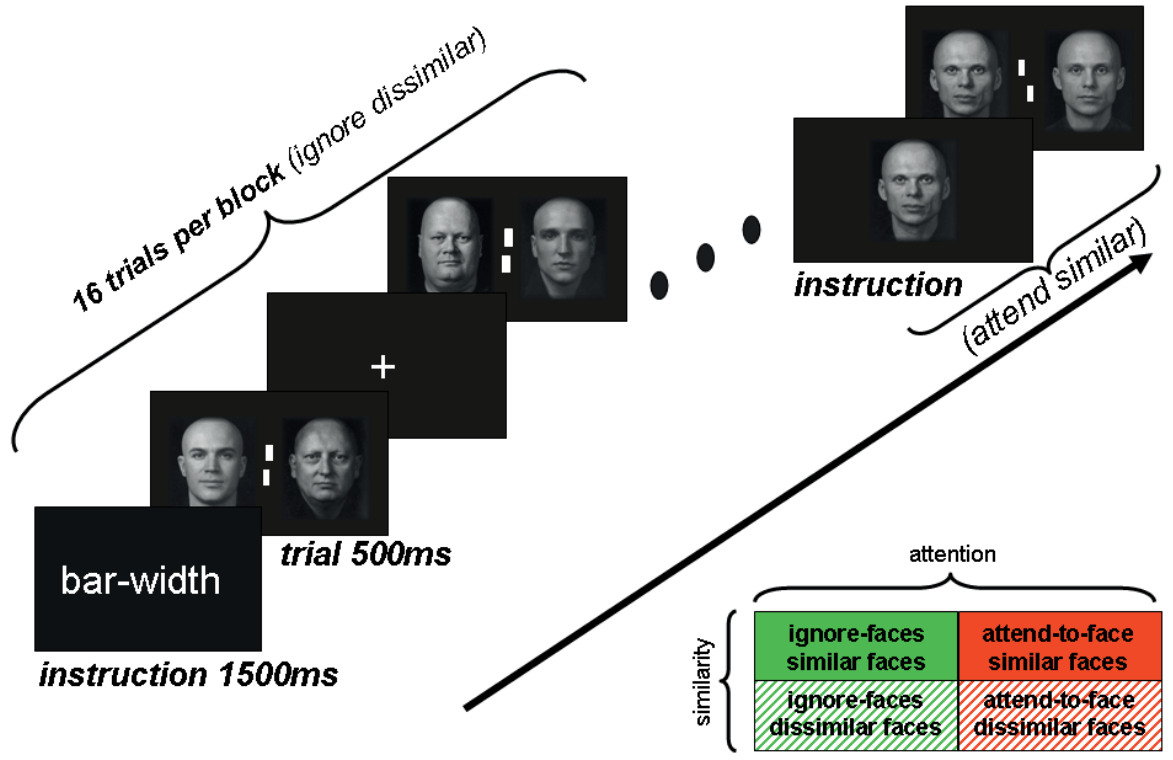

Figure 2. Experimental design. Block structure with examples of two different conditions (ignore-faces with dissimilar display and attend-to-face with similar display).

The design was a $2 \times 2$ repeated measures design with factors task (ATT, IGN) and similarity (SIM, DISS) leading to 4 conditions (IGN_DISS, IGN_SIM, ATT_SIM, ATT_DISS, Figure 3.b). The participants were not made aware of the similarity manipulation. The order of the blocks was randomised and counterbalanced across participants and runs by means of Williams design (Williams EJ, 1949). This scheme is a generalized Latin square design, balanced for first-order carryover effects. The stimulus display was identical for both types of task. Participants were instructed to fixate the centre of the screen throughout the experiment and to minimise head and eye-movements while in the scanner. 
a)

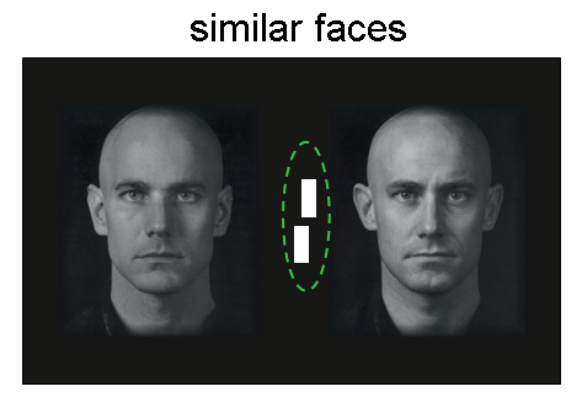

ignore-faces

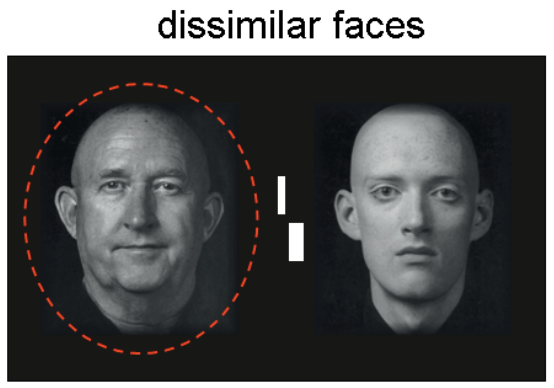

attend-to-face

b)

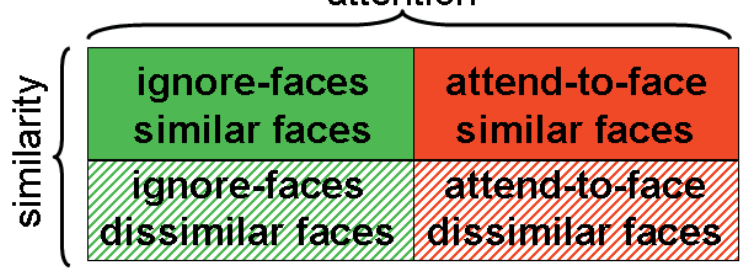

Figure 3. Tasks and stimuli. (a) Examples of the two types of the display presented (similar and dissimilar faces) for the two tasks (ignore-faces and attend-to-face). (b) Conditions: $2 \times 2$ repeated measures design.

\subsection{Data acquisition}

Images were acquired on a 3T Siemens Magnetom Allegra head scanner (Siemens Medical System, Erlangen, Germany) using a standard head coil. Thirty-two oblique axial slices (inplane resolution: $3.5 \mathrm{~mm} \times 3.5 \mathrm{~mm}$, slice thickness: $3.5 \mathrm{~mm}$, interslice distance $0 \mathrm{~mm}$ ) covering the entire cortical volume were acquired using an echo planar imaging sequence (repetition time $(T R)=2000 \mathrm{~ms}$, echo time $(T E)=29 \mathrm{~ms}$, matrix: $64 \times 64)$. We acquired 612 volumes per run and discarded the first two from the analysis due to the T1 saturation effect. Functional slices were aligned to a high resolution 3D anatomical dataset acquired in the middle of the entire session and consisting of 176 slices (for 15 subjects the MDEFT sequence was used: $\mathrm{TR}=7.92 \mathrm{~ms} ; \mathrm{TE}=2.4 \mathrm{~ms}$; flip angle $=15^{\circ}$, voxel dimension $=1 \times 1 \times 1 \mathrm{~mm}^{3}$; due to better performances the ADNI sequence replaced the MDEFT sequence for 4 subjects: TR = $2250 \mathrm{~ms} ; \mathrm{TE}=2.6 \mathrm{~ms}$; flip angle $=9^{\circ}$, voxel dimension $=1 \times 1 \times 1 \mathrm{~mm}^{3}$ ).

The participants were placed comfortably in the scanner and their head was fixated with foam pads. Participants saw the stimuli projected on a screen through a mirror mounted on the head coil. The visual field was perceived at a distance of 57 centimeters. The stimuli were delivered using the Presentation 12.2 package (Neurobehavioral Systems, 
Inc. www.neurobs.com). Stimulus presentation was synchronized with MR data acquisition by triggering the stimulus program with the first MR pulse.

\subsection{Analysis}

Both the functional and the anatomical data were analyzed using the BrainVoyager QX package (Brain Innovation B.V., Maastricht, The Netherlands). The anatomical scans were used to project the statistical results from the functional data onto high-resolution anatomical images. They were also used to derive the Talairach coordinates needed to normalize both sets of data. Functional data were pre-processed, aligned to the anatomical images, and normalized. The pre-processing procedure started with correcting the data for motion artefacts in three dimensions and for slice scan-time differences. Subsequently, linear drifts were removed from the signal and data were high-pass filtered to remove slow frequency drifts up to 9 cycles per time course. After the pre-processing, functional data were aligned to the high-resolution anatomical images and normalized to the standard 3-dimensional Talairach space. The final version of the functional data consisted of a 4-dimensional $(x, y, z, t)$ dataset in Talairach space for each run and participant.

After brain normalization, the statistical analysis from multiple subjects was performed via the General Linear Model (GLM). In the main experiment, a random effects (RFX) approach was used with subject as a random variable. The RFX-GLM analysis was implemented via multi-level summary statistics. In a first level, a standard GLM analysis was used to estimate the beta values (summary statistics) for each subject. These values were entered as dependent variables in the second level analysis. At the second analysis level full factorial effects (main effects and $2 \times 2$ interaction effect) were tested. These effects were tested in conjunction with contrasting all the conditions versus baseline. The statistical analysis was carried out in a region of interest (ROI), which was specified by an independent functional localizer (FFA, see below), and in the whole brain.

For the whole-brain analysis, correction for multiple comparisons was performed using cluster-size thresholding. The procedure started with thresholding the map relative to the contrast of interest with $t=3.15(p=.007)$ as suggested by Goebel et al. $(2006)$ at the voxellevel. Next, 1,000 normally distributed noise images were generated by randomly selecting values from a normal distribution (Monte Carlo simulation), and the noise map was smoothed using an estimate of the intrinsic smoothness of the source statistical map (Forman SD et al., 1995; Goebel R et al., 2006). The noise images were thresholded at the pre-defined voxel-level threshold. Clusters of voxels surviving the threshold were tabulated according to cluster size. The final distribution of cluster sizes was thresholded against a false-positive rate of 0.05 . The minimum cluster size that survived the thresholding was used as cluster-size threshold of the source statistical map. This yielded a multiple comparison correction of $p=0.05$ at the cluster-level. The correction for multiple comparisons was not performed for the ROI analysis in FFA. In fact, in this case, the beta values of the fMRI signal 
per subject and condition were extracted, averaged over all voxels in the single-subject volume-of-interest (VOI), and evaluated (summary statistics, level 1).

Statistical maps were projected on an average brain, which was computed by averaging normalized cortices from all the subjects. The normalization of the cortices was achieved via cortex-based alignment. This procedure aligns the individual cortical renderings by increasing the overlap of curvature information of the cortices (Fischl B et al., 1999; Goebel R et al., 2006).

\subsection{Localization of FFA}

An independent functional run, consisting of 324 volumes, was used to localize FFA in all subjects. The scanner-parameters used to acquire the data were the same used for the main experimental runs. The stimuli used to localize the face-processing-area consisted of pictures of faces, scrambled faces, objects, and places (Goffaux $V$ et al., 2009). The scrambled faces were obtained by applying the Fourier phase randomization procedure (Nasanen $R, 1999$ ) to the original set of faces. The algorithm for "scrambling" faces consisted of two steps: each picture from the set of faces was first Fourier-transformed. Subsequently, the phase spectrum of the resulted transform was replaced by random values, while keeping the amplitude spectrum of the image unaltered. This procedure yielded images that preserved the global low-level properties of the original image (luminance, contrast, spectral energy, etc.), and degraded completely any category-related information. The 4 different categories of stimuli were presented in blocks ( 20 stimuli per block) for 4 times. In order to keep the attention of the subject constant across the run, participants were asked to indicate with a button-press whether the stimulus colour changed from a typical black-white to a light red.

After the acquisition, the functional data were pre-processed (in this case, slow frequency drifts were removed up to 3 cycles) and normalized following the same procedure previously described for the main experimental runs. For the statistical analysis of the FFA localizer, a FFX-GLM analysis was performed with the four stimulus types as predictors. The condition faces was contrasted with all the others. Finally, FFA was localized considering the clusters of voxels, within the fusiform gyrus, statistically significant (FDR-corrected, $q=.05$ ). For 2 subjects (out of 16 analyzed) the left hemisphere did not reveal any FFA localization. Therefore, for the main experiment, the ROI analysis was performed for 16 subjects in the right FFA and for 14 subjects in the left FFA (Table 1). 


\section{Results}

\subsection{Behavioral Results}

We computed error rates (ERs) and reaction times (RTs) on the ignore-faces (IGN) and attend-to-face (ATT) task for similar (SIM) and dissimilar (DISS) condition. Mean ERs and RTs were then submitted to a repeated measures ANOVA with two factors, namely attention and similarity. For RTs, no significant main effects of attention, similarity or interaction were observed. Attention: $(\mathrm{F}(1,14)=3.99, \mathrm{p}=.066$, mean reaction time ATT $=629 \mathrm{~ms}, \mathrm{SE}=18$, mean IGN = $605 \mathrm{~ms}$, SE = 17). Similarity: $(\mathrm{F}(1,14)=2.5, \mathrm{p}=.136$, mean SIM $=619 \mathrm{~ms}, \mathrm{SE}=$ 16 , mean DISS $=614 \mathrm{~ms}, \mathrm{SE}=16)$. Interaction: $(F(1,14)=3.18, p=.096)$. The same holds for error rates. Attention: $(F(1,14)=4.52, p=.052$, mean ATT $=27 \%$, SE $=4$, mean IGN $=18 \%$, $\mathrm{SE}=1)$. Similarity: $(\mathrm{F}(1,14)=.01, \mathrm{p}=.919$, mean $\mathrm{SIM}=22 \%, \mathrm{SE}=2$, mean DISS $=23 \%, \mathrm{SE}=$ 3). Interaction: $(F(1,14)=.00, p=.957)$.

\subsection{Eye-Movements}

We used the data recorded in a parallel EEG experiment with an identical procedure (instruction, display, task and timing) to monitor for eye-movements. The electroencephalogram (EEG) was measured in 18 different subjects using tin electrodes (32 channels in total, QuickCap, Neuroscan Inc. Tm). Reference electrodes were positioned at both mastoids (A1, A2). The left mastoid (A1) was used as an online reference for the scalp sites. Horizontal eye movements were recorded with a bipolar montage placed at the left and right canthi (HEOG). Electrode impedance was kept below $5 \mathrm{~K} \Omega$. EEG data were recorded and amplified using Neuroscan SynAmps with a $0.05-30 \mathrm{~Hz}$ bandpass and sampled at 4-ms intervals (250 $\mathrm{Hz}$ ). The EEG signal was epoched with an interval of $900 \mathrm{~ms}$, time-locked to the onset of the presentation of the pair of faces. Epochs included a 100-ms prestimulus baseline correction interval and they were $0.3-30 \mathrm{~Hz}$ bandpass filtered (Butterworth, zero phase, 24dB/oct). Horizontal eye movements were defined as visible step functions in the HEOG signal change. The amount of horizontal eye movements was on average below $4 \%$ of the total amount of trials for all conditions, and they did not differ significantly for the two tasks (attend-to-face, ignore-faces) ( $F=3.91, p=.065)$. In practice, one would expect a larger amount of horizontal saccades within the attend-to-face related to overt shifts of attention, this directionality would lead to a one-tailed $p$ of .065/2 ( $p=.032$ ). This means that in $4 \%$ of the cases, a difference in eye movement cannot be ruled out. The same analysis performed within the ignorefaces condition did not show any difference in the amount of eye movement between the ignore-similar-faces and ignore-dissimilar-faces condition ( $F=.81, p=.379)$, indicating that eye movement most likely do not play a role in explaining the observed similarity effect in this condition. 


\subsection{ROI analysis in FFA}

A RFX-GLM analysis restricted to FFA (Figure 4, Table 1) revealed, bilaterally, a significant interaction of factors attention (ATT vs. IGN) and similarity (SIM vs. DISS) (right FFA: $p=.017$, left FFA: $p=.045$ ), a significant main effect of attention (ATT $>$ IGN) (right FFA: $p=.000$, left FFA: $p=.000$ ) and no main effect of similarity. The interaction, qualitatively visible also in the time course in Figure 4, reflected a larger fMRI signal when dissimilar faces were presented compared to similar faces in the ignore-faces condition, both in the right and left FFA (IGN_SIM < IGN_DISS) (right FFA: $p=.011$, left FFA: $p=.003$ ). No significant differences between SIM and DISS displays within the attend-to-face conditions (ATT_SIM = ATT_DISS) were observed in either hemisphere, based on simple effect analysis.

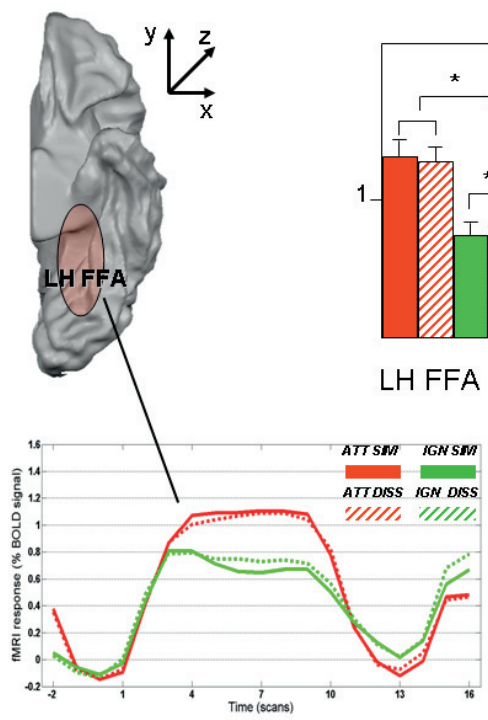

beta values

$\star=p<0.05$

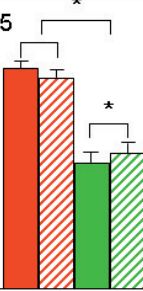

RH FFA

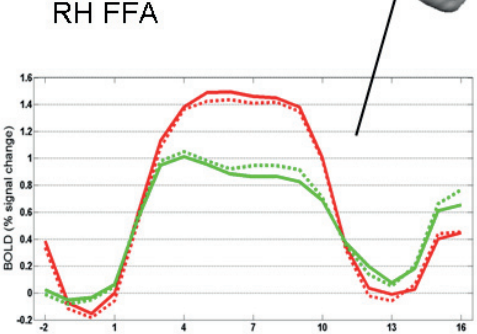

Figure 4. Results of the RFX-GLM analysis in FFA (region of interest analysis) separately for left and right hemisphere. In the central column the beta weights of the GLM analysis are displayed. The mean time courses of the fMR signal per condition and per region of interest are also shown. 


\subsection{Whole-brain GLM analysis for Visual areas}

The whole-brain GLM analysis (Figure 5, Table 1) revealed an interaction of factors attention (ATT vs. IGN) and similarity (SIM vs. DISS) ( $p=.007$, corrected) also in the inferior occipital sulcus (corresponding to the lateral occipital sulcus in Talairach coordinates). Such a region has recently been associated with face processing and was identified as the occipital face area (OFA) (Dubois S et al., 1999; Gauthier I et al., 2000; Halgren E et al., 1999; Haxby JV et al., 1999; Hoffman EA and JV Haxby, 2000; Kanwisher N et al., 1997; Rossion B et al., 2003). A post-hoc analysis restricted to OFA revealed a main effect of attention in the left hemisphere $(p=.005)$. The main effect of attention was significant $(p=.007$, corrected) also in the inferior part of the right OFA, in the primary visual cortex, the posterior part of the collateral sulcus and within the temporal part of the fusiform gyrus.

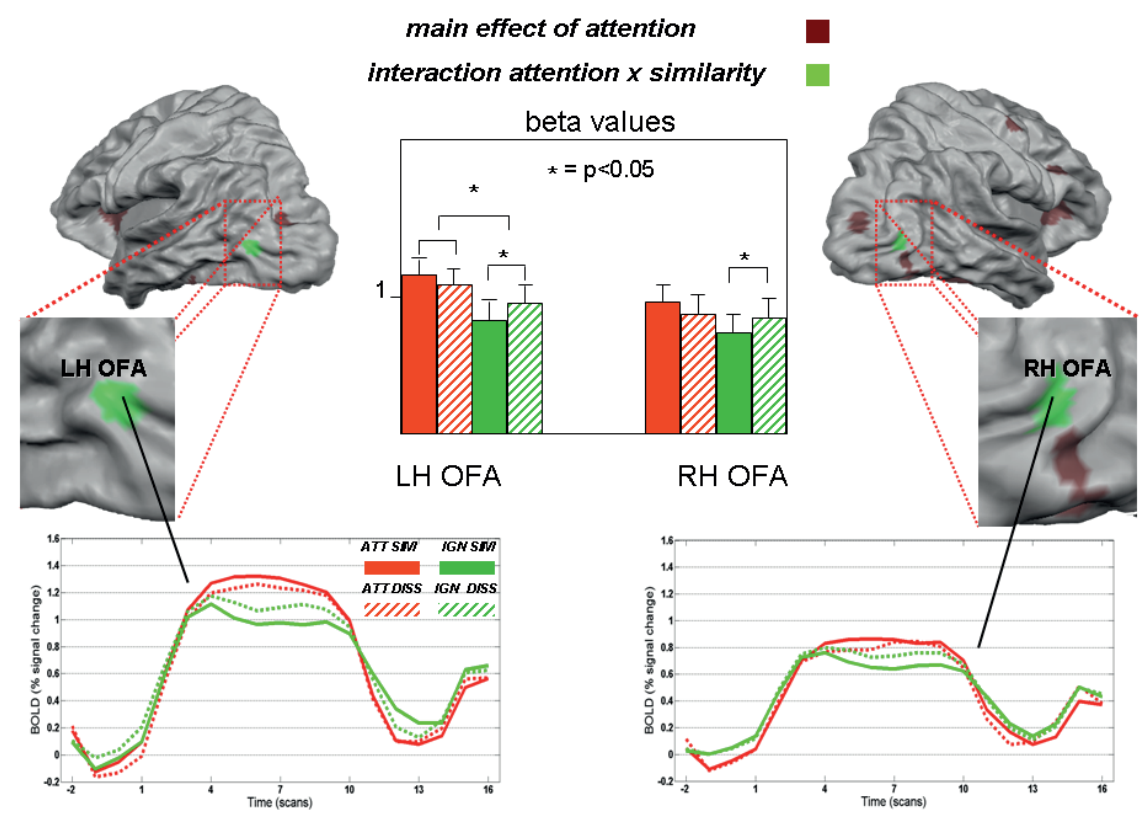

Figure 5. Results of the RFX-GLM analysis in OFA (whole brain analysis), separately for left and right hemisphere. In the central column the beta weights of the GLM analysis are displayed. The GLM beta weights are referred to the part of OFA showing an interaction effect. The mean time courses of the fMRI signal per condition and per region of interest are also shown. 
Within the frontal lobe we distinguished several areas showing a main effect of attention (Figure 6, Table 1). In particular, the contrast ATT > IGN revealed bilateral activations within the insula ( $p=.007$, corrected). Lateralized uniquely on the right hemisphere we found clusters of activations for the same contrast ( $p=.007$, corrected) within the inferior frontal gyrus (IFG), the inferior part of the precentral sulcus and within the medial part of the precentral gyrus. The main effect of similarity or interaction of factors was not significant in any of these or other regions within the frontal lobe.

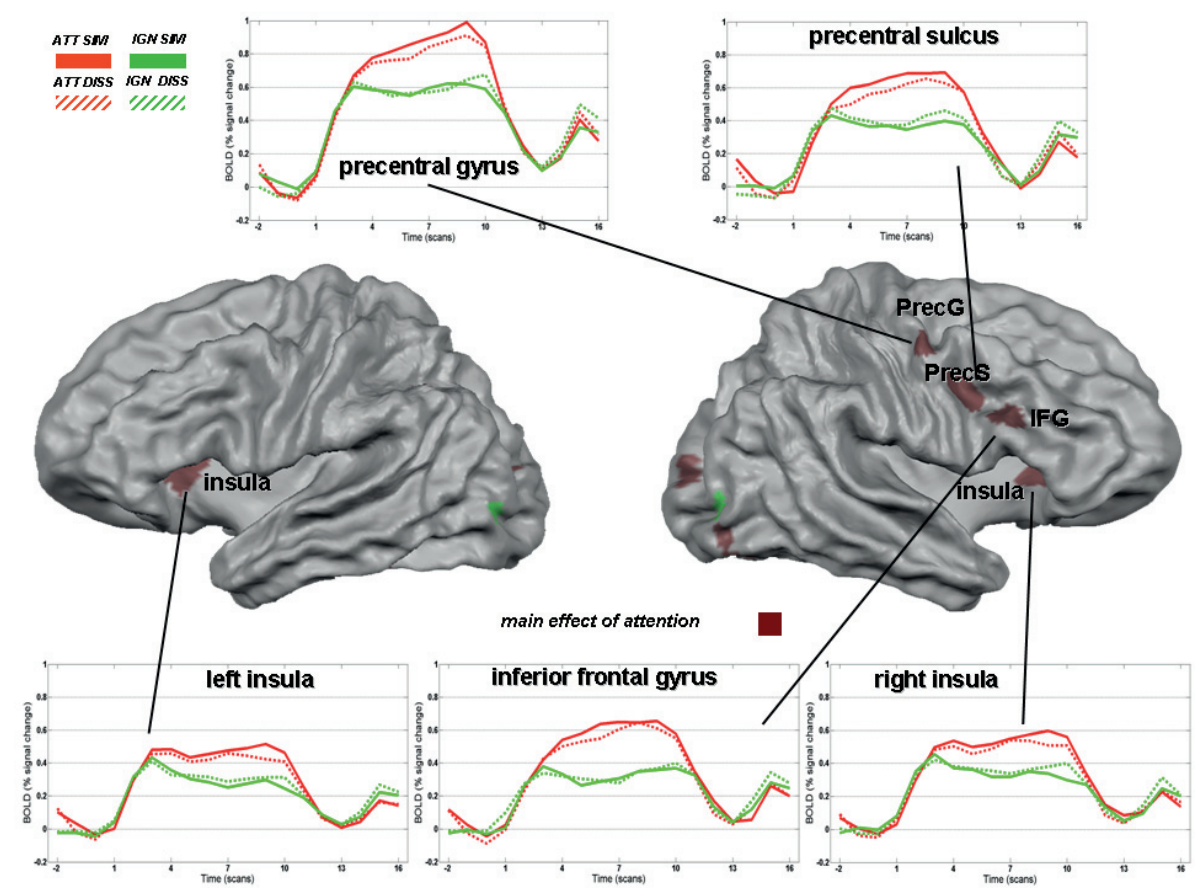

Figure 6. Results of GLM analysis in frontal areas (whole brain analysis), separately for left and right hemisphere. The mean time courses of the $\mathrm{fMRI}$ signal per condition and per region of interest are displayed. The mean time courses related to activations in the occipital-temporal lobe are not shown as they were already underlined in Figure 5. 


\begin{tabular}{|c|c|c|c|c|c|c|}
\hline \multirow{3}{*}{$\begin{array}{l}\text { Area } \\
\text { FFA }\end{array}$} & \multicolumn{3}{|c|}{ Right hemisphere } & \multicolumn{3}{|c|}{ Left hemisphere } \\
\hline & $\mathrm{x}$ & y & & $\mathrm{x}$ & $z$ & \\
\hline & $37 \pm 3$ & $-46 \pm 7$ & $-19 \pm 4$ & $-39 \pm 3$ & $-45 \pm 6$ & $-18 \pm 3$ \\
\hline OFA & 40 & -75 & -3 & -40 & -78 & -3 \\
\hline PrecG & 44 & -12 & 52 & & & \\
\hline PrecS & 38 & -1 & 36 & & & \\
\hline $\mathrm{IFG}$ & 45 & 14 & 30 & & & \\
\hline Insula & 32 & 22 & 12 & -30 & 17 & 13 \\
\hline
\end{tabular}

Table 1. Talairach coordinates related to all regions of interest. Note that as the left and right FFA was localized respectively for 14 and 16 subjects, individually, the mean Talairach coordinates for this area are displayed with the standard deviation.

\section{Discussion}

In the present Chapter we investigated the mechanism of face selection in the context of the $\mathrm{BC}$ model of attention, using $\mathrm{fMRI}$. According to $\mathrm{BC}$, neural representations of irrelevant (ignored) faces engage in mutual suppressive interactions, whereas attention to a particular face biases the competition towards the processing of this face only. Participants were presented with blocks of similar or dissimilar pairs of faces and were asked to attend to one of these faces (attend-to-face condition) or ignore both of them (ignore-faces condition). The direct comparison between the attend-to-face vs. ignore-faces condition was used to estimate the occurrence of competitive interactions between simultaneously encoded faces. Neural competition in the ignore-faces condition was investigated in more detail by comparing conditions where similar and dissimilar pairs of faces were presented. The similarity manipulation was also used to test the hypothesis that a higher overlap in representation (for similar faces) corresponded to a higher level of competition. 
In line with the $\mathrm{BC}$ model, we observed a lower $\mathrm{fMRI}$ signal when the two faces were ignored compared to when one of them was attended to. In addition, and most interestingly, we found an attention $x$ similarity interaction effect in FFA and OFA suggesting that the similarity manipulation influenced neural activations only within the ignore-faces condition but not in the attend-to-face condition. The direction of the similarity effect in the ignore-faces condition (similar < dissimilar) can be interpreted within the framework of BC and neural population coding: similar faces compete for representation more than dissimilar faces because they are encoded by neurons that are spatially clustered together.

In line with the bias effect of the $\mathrm{BC}$ model we also observed that the enhancement of activation observed during the attend-to-face condition was independent of the similarity level between the faces (similar = dissimilar). We interpreted these results as support for the $\mathrm{BC}$ model for face selection within face selective areas. Finally, results in frontal areas suggested that the attentional bias might be controlled by the precentral gyrus/sulcus and the IFG.

\subsection{Neural competition and similarity modulation in FFA and OFA}

In the classical BC paradigm, neural competition in early visual areas between two stimuli was normally tested by comparing the activation of those items presented alone with the same items presented simultaneously. However, the higher amount of transients for single faces presented in sequence compared to pairs of faces presented simultaneously could give rise to an overestimation of neural competition occurring in the latter condition. The attendto-face condition, in contrast, had the same amount of transients as the ignore-faces condition, and hence, offers an alternative way to study BC. In addition, cell recording and fMRI studies found that, when attention was directed to one of the items presented in a multiple display, the neural response increased up to the level of the response to the single item presented alone (Recanzone GH, 2000; Reynolds JH et al., 1999). A recent fMRI study confirmed this idea in the face perception domain as it showed that the attend-to-face condition is a suitable baseline for single-face processing (Reddy $L$ et al., 2009).

Therefore, in order to overcome the problem of differences in stimulation onsets, we used the comparison attend-to-face vs. ignore-faces condition to investigate neural competition in FFA. We also relied on the assumption that FFA is sensitive to faces presented in two different hemifields (Halgren E et al., 1999; Reinholz J and S Pollmann, 2007) and that, in turn, both faces were encoded and compete for representation (Desimone R, 1998; Desimone R and J Duncan, 1995; Kastner S and LG Ungerleider, 2000, 2001; Pessoa L et al., 2003; Reynolds JH and R Desimone, 1999). As expected, the fMRI signal was lower when both faces in the pair were ignored compared to when one of them was attended to (ignore-faces < attend-to-face) suggesting that two faces simultaneously presented mutually interacted with each other in a suppressive manner. However, this difference between the two tasks could also be related to an enhancement effect due to spatial attention. 
In order to confirm the hypothesis that faces in a pair competed for representation, we focused on the influence of the similarity manipulation within the ignore-faces condition. In line with the neural competition effect, we observed that the similarity level between the two faces played a role only within the ignore-faces and not in the attend-to-face condition. In particular, the ROI analysis in FFA revealed a significant interaction of the factors attention (ATT vs. IGN) and similarity (SIM vs. DISS) and a significant modulation by similarity within the ignore-faces condition. In line with our predictions, the fMRI signal in FFA was smaller for similar than for dissimilar faces when both faces were ignored. We interpreted this result as reflecting greater neural competition between similar faces. A vast line of research investigated the spatial organization in primate inferior temporal (IT) cortex and its functional role in object recognition. Several electrophysiological and optical imaging experiments revealed a general tendency for similar complex features to be mapped in nearby locations in IT (Fujita I et al., 1992; Kreiman G et al., 2006; Sato T et al., 2008; Tamura H et al., 2005; Tanaka $\mathrm{K}, 2003$; Wang G et al., 1998). In humans, two fMRI experiments confirmed these findings in the context of face processing (Jiang X et al., 2006; Loffler $G$ et al., 2005). Both studies used an $\mathrm{fMRI}$ adaptation ( $\mathrm{FMRI}-\mathrm{A}$ ) paradigm to infer functional properties of neural populations at subvoxel resolutions (Grill-Spector $\mathrm{K}$ et al., 2006). Results from both studies suggested that similar faces were encoded by the same neural population while dissimilar faces were encoded by different subpopulations.

The proposed spatial organization of face selective areas may play a role during neural competition among simultaneously presented stimuli. In fact, the different level of similarity between faces in our experiment would draw upon different spatial distributions of neurons encoding both faces in FFA. In particular, we assumed that similar faces would share their neural representations within the same neural population and they would be seen as ambiguous input at the neural level. This would imply in the context of $\mathrm{BC}$ that two similar faces simultaneously presented compete more for representation than dissimilar faces. The higher amount of competitive interactions, in the case of similar faces, should result in a lower fMRI signal in FFA. The significant pairwise comparison IGN_SIM < IGN_DISS supported this hypothesis. In a more general context, this finding of a visual similarity effect also indicated that both faces were processed by FFA to some extent when presented at the same time even though ignored.

We observed the same results in OFA as we found in FFA. According to the model of face perception of Haxby et al. (2000) face information is processed within two stages. In the first stage, individual facial features and specific attributes as age or gender are extracted (Bruce V and A Young, 1986; Sergent J et al., 1992). Information from the first stage is sent further to the second processing stage, in which global face configuration is achieved. The first processing stage has been associated with OFA, which sends information along the ventral stream to FFA, which has been associated with the second processing stage. Based on this model, we can conclude that neural competition between face representations occurred also at the level of single face features in OFA. 
Next to neural coding, perceptual grouping may also play a role in explaining the data in the context of BC. More specifically, an interaction of grouping and $B C$ might account for the similarity modulation we observed in FFA and OFA in the ignore-faces condition (interaction of attention $\mathrm{x}$ similarity). In fact, we might assume that similar faces form a perceptual group according to the Gestalt laws whereas dissimilar faces do not. In a review on BC, Reynolds and Desimone (1999) proposed a link between neural competition and perceptual grouping. They claimed that items forming perceptual groups are encoded within the same receptive field. Therefore, as competitive interactions are stronger at the level of receptive field, two similar faces (grouping by similarity) were more likely to compete for representation than dissimilar faces, leading to a lower fMRI signal. In the same review, Reynolds and Desimone also suggested that attending to one stimulus cause the RF of a cell to "shrink" around the attended item only. This in turn would explain the absence of similarity modulation within the attend-to-face condition. However, for the current study we consider the grouping interpretation as rather unlikely because of the low amount of stimuli (only two faces), their relatively large size $\left(6.5^{\circ} \times 5.4^{\circ}\right.$ each) and their position in the visual field (two opposite hemifields).

As an alternative to the $\mathrm{BC}$ account, our result could be related to neural adaptation. Several $\mathrm{fMRI}$ studies have shown a decreased response following stimulus repetition. This effect has normally been tested by presenting sequences of similar or identical items. In our study we also mentioned two fMRI studies that performed an adaptation design using similar faces (Jiang X et al., 2006; Loffler $G$ et al., 2005). Both experiment showed a reduced activity when similar faces were presented compared to dissimilar faces, demonstrating that FFA might be functionally organized according to the similarity level of the faces represented. Three models have been proposed as a neural basis for the adaptation effect. The fatigue model assumes that the same neurons activated during the first presentation are also activated but less strongly when the stimulus is presented again. In the sharpening model, neurons become specialized and during the repetition a smaller percentage of neurons activates compared to the first stimulus presentation. Finally, the facilitation model predicts that repetition of a stimulus causes faster processing of the stimulus repeated which leads a neural activity of shorter duration and/or latencies (Grill-Spector $\mathrm{K}$ et al., 2006). To our knowledge, none of these neural causes have been related to the effects normally observed when several items are simultaneously presented. In addition the present experiment was carefully designed in order to avoid any adaptation effect due to repetition of similar faces. In fact, the similarity level between faces was manipulated only at the level of single trial (within a pair) and not across trials. Pairs of faces in a block were different to each other both in the similar and dissimilar condition such that any type of adaptation across conditions was kept constant. However, due to the limited understanding of human neural adaptation, we cannot strictly rule out that similar mechanisms (fatigue, sharpening, or facilitation) are effective in sequential as well as in simultaneous presentation. 
A third possible interpretation of the results in the ignore-faces condition could be that neurons processing one of the faces were inhibited via lateral local connections by surrounding neurons encoding the other face. This, in turn, would result in a higher inhibition effect for similar compared to dissimilar faces (Knierim JJ and DC van Essen, 1992).

\subsection{Attentional bias in FFA and OFA}

The involvement of spatial attention to one of the faces elicited a general enhancement of hemodynamic activity compared to the line comparison task (Wojciulik E et al., 1998). Furthermore, the enhancement we observed when attention was shifted to one of the faces was statistically indistinguishable when the distractor was similar or dissimilar to the target face. Therefore, attention to the target face seems to increase the effective "spatial resolution" of the visual system in order to process only the attended face among the two presented (Reynolds JH and R Desimone, 1999). Moreover, the same level of activation between the two attend-to-face conditions confirmed that single faces in both similar and dissimilar sets were properly selected to elicit the same fMRI signal in FFA. It is unlikely that the increased activation in FFA and OFA was due to a higher level of difficulty of the attendto-face task compared to the ignore-faces task. In fact, the behavioral results showed no significant differences in reaction time and error rates between the two tasks. The amount of eye movement recorded in an EEG experiment using the same experimental design partially confirmed these results. In fact, the total amount of horizontal saccades was very low $(<4 \%)$ and a two-tailed ANOVA did not show any difference between the two tasks (attendto-face, ignore-faces conditions). However, if we assumed that in the attend-to-face task subjects had the tendency to make horizontal eye-movements towards the face on the left, a one-tailed ANOVA was the proper statistical test to perform. This analysis revealed a significantly larger amount of horizontal saccades for the attend-to-face vs. ignore-faces condition. Therefore, we cannot fully rule out the possibility that the attentional effect we found in OFA and FFA was not related to eye-movement.

In summary, in line with the BC model, results in FFA and OFA suggested that the two simultaneously presented faces were encoded and competed with each other in these areas (attend-to-face > ignore-faces, ignore-similar-faces < ignore-dissimilar-faces). In addition, we observed that attention solved neural competition by biasing the neural processing towards the target face only (attend-to-face > ignore-faces, attend-to-similar-face = attend-todissimilar-face).

\subsection{Attentional Bias via Frontal areas}

Results in visual areas showed that when one of the faces in the visual field was attended to, fMRI signal increased compared to the neural response during the ignore-faces condition. The whole brain analysis revealed possible frontal sources of this biasing signal. In particular, 
the insula, IFG, precentral sulcus and precentral gyrus were selectively recruited during the attentional task. In the present attentional task the subjects attended to the face on the left and performed a match-to-face task. The task was performed while ignoring a distracting face on the right. Previous studies involving similar attentional tasks found an increase of activation in ventrolateral prefrontal regions (Gitelman DR et al., 1999; Hopfinger JB et al., 2000; Thomsen T et al., 2005). According to the authors these areas were involved in the inhibitory filtering of the distracting item in the hemifield opposite to the focus of attention. In a slightly different view Brown et al. (2006) proposed that both the insula and IFG are needed to keep attention captured while inhibiting any shift of attention towards the distracting location. Our results for the insula and IFG might reflect both mechanisms. In fact, in order to perform the task, subjects optimized their focus on the "target" position (left) by suppressing any attentional bias to the right. Activation in the insula during an attentional task might also reflect a higher level of anxiety or alertness, as proposed by Lawrence et al. (2003) in a rapid visual information processing paradigm.

The right precentral sulcus and gyrus also showed a higher fMRI signal during the attentional task compared to the ignore-faces task. Although the precentral sulcus has been demonstrated to be recruited during generation of eye movement, several studies showed that this area is also involved in visuospatial attentional processes (Brown MR et al., 2006; Corbetta M et al., 1998; Gitelman DR et al., 1999; Grosbras MH et al., 2005; Hopfinger JB et al., 2000; Law I et al., 1997; Nobre AC et al., 1997; Thomsen T et al., 2005) and attentional control (Pessoa L et al., 2003). This versatile nature of the precentral sulcus was already hypothesized by Rizzolatti et al. (1987) within the premotor theory of attention. They stated that covert attentional mechanisms and overt saccade generation share a neural circuitry in the initial stages of processing.

Together with the precentral sulcus, the precentral gyrus is also part of the network involved in saccadic eye movement and covert attention (Grosbras MH et al., 2005; Hopfinger JB et al., 2000; Law I et al., 1997; Petit L et al., 1999). In summary, the right precentral sulcus/gyrus and IFG seem to be plausible candidates to be involved in the bias of neural competition in face selective areas. Overall, this study showed for the first time that BC may play a relevant role in FFA and OFA for face selection.

\section{Acknowledgments}

The authors wish to thank M. Bles for helpful discussions. This project was supported by NWO ASPASIA grant nr. 015.001.053 and the Maastricht University HRM Policy “Mobilizing minds" (increase of females in higher positions). 


\section{Temporal dynamics of face selection} mechanism in the context of face processing $^{1}$

\footnotetext{
${ }^{1}$ Corresponding publication: Gentile F and Jansma BM, Temporal dynamics of face selection mechanism in the context
} of similar and dissimilar faces: ERP evidence for local biased competition within FFA using ICA (submitted) 


\begin{abstract}
This study was designed to investigate the spatio-temporal characteristic of face selection in the context of the Biased Competition (BC) model using electroencephalography (EEG). Pairs of similar (SIM) or dissimilar (DISS) faces were presented simultaneously. Subjects had to attend to one face (ATT) or ignore both faces (IGN). According to the BC account, simultaneously presented faces compete for representation. Spatial attention biases these competitive interactions towards neural processing of the attended face alone. A preceding functional magnetic resonance imaging ( $\mathrm{fMRI}$ ) study confirmed the validity of $\mathrm{BC}$ as selection principle in the occipital face area (OFA) and in the fusiform face area (FFA) (IGN SIM<IGN DISS; ATT SIM=ATT DISS) (Gentile F and BM Jansma, 2010). The directionality of the similarity modulation within the IGN condition was interpreted in line with the assumption that within FFA similar faces are more likely encoded by the same population of neurons, hence leading to a stronger competition. In the present EEG experiment we investigated when neural competition and the bias effect take place. Using independent component analysis (ICA), we focused on face-processing-related components N170 and N250. Whereas we could not find any context-dependent modulation of the N170, we observed a context $x$ task interaction within the N250 time-window. Together, the fMRI and EEG results suggested that faces competed for representation (IGN SIM<IGN DISS) in the N250 time-window within FFA. In addition, at the same latency (250ms post-stimulus onset) and region of interest (FFA) the data supported the idea that attention solved neural competition in favor of the relevant face (ATT SIM=ATT DISS). We also observed a main effect of attention frontally within the $\mathrm{N} 2 \mathrm{~b}$ time-window, i.e. about 50 ms later than the attentional bias that solved competitive interactions. Based on these findings, it is plausible to assume that the frontal activity was not directly involved in the bias control during selection in FFA.
\end{abstract}

Keywords: Biased Competition, face selection, visual similarity, N170, N250, ICA 


\section{Introduction}

Due to its limited capacity, the visual system is not able to fully process several stimuli at the same time. Attentional mechanisms are needed to select the task-relevant item in a clutter of other objects (Broadbent D, 1958; Carrasco M et al., 2004; Desimone R and J Duncan, 1995; Hawkins HL et al., 1990; Luck SJ et al., 1994; Treisman AM, 1969). According to the classical view, attention works as a "spotlight" that enhances the neural representation of the attended stimulus (Brefczynski JA and EA DeYoe, 1999; Hillyard SA and TF Munte, 1984; Luck SJ et al., 1994; Posner MI et al., 1980; Reynolds JH et al., 2000). The influence of attention on visual processing was further elaborated by models of attention that assumed a competitive interaction among items simultaneously presented and ignored. The most influential one is the Biased Competition (BC) model. According to $B C$, when two stimuli are presented at the same time within the receptive field of a neuron, the neural representations of the two items interact in a mutually suppressive manner. In this framework, attention acts to bias the neural competition between the two competing items in favor of the neural processing of the attended one (Desimone R, 1998; Desimone R and J Duncan, 1995; Kastner S and LG Ungerleider, 2000, 2001; Pessoa L et al., 2003; Reynolds JH and R Desimone, 1999).

Several electrophysiological studies tested this model in monkeys. The classical design consisted of presenting simultaneously two different items within the receptive field of the recorded cells in the visual cortex, while the monkey ignored the stimuli. In such an experimental setting the cells' firing rate related to the objects was a weighted average of the activity of the same items presented alone. In line with the $B C$ model, these results suggested that multiple objects compete for representation. In the context of neural competition, the effect of attention was also investigated. The neural activity associated with attending to one item in a group of other objects was comparable to the activity related to the processing of the same items presented alone and being ignored (Recanzone GH, 2000; Reynolds JH et al., 1999). These results confirmed the role of attention as the bias control needed to select the stimulus relevant for behavior (Chelazzi L et al., 1998; Chelazzi L et al., 2001; Desimone R and J Duncan, 1995; Luck SJ et al., 1997; Miller EK et al., 1993; Moran J and R Desimone, 1985; Reynolds JH et al., 1999; Treue S and JC Martinez Trujillo, 1999; Treue S and JH Maunsell, 1996). According to Reynolds and Desimone (1999), the competing interactions between stimuli representations are caused by the inhibitory connections projecting from the cells encoding the stimuli to the recorded neuron. In addition, attention to one of the stimuli suppresses the inhibiting influence of the unattended stimulus. This in turn makes the attended stimulus the only one that is processed as relevant.

The BC model was also tested in the human visual cortex with functional magnetic resonance imaging $(\mathrm{fMRI})$. A first series of experiments focused on the competitive aspect of 
BC. They showed that when several items were simultaneously presented the fMRI signal decreases compared to when they were presented one at a time. This result was interpreted as a suppression effect due to neural competition among the items in the cluster (Beck DM and S Kastner, 2005, 2007; Kastner S et al., 1998; Kastner S et al., 2001; Kastner S et al., 1999). The attentional bias hypothesis of the BC model was approached by another series of studies. They observed a general enhancement of activation when both top-down and bottom-up attentional mechanisms came into play. In particular, the fMRI signal increased up to the level of the activation elicited by the same objects presented alone whenever one stimulus in a cluster of other stimuli was attended to (Beck DM and S Kastner, 2005; Bles M et al., 2006; Kastner S et al., 1999).

A recent fMRI experiment by Reddy et al. (2009) tested the BC model by presenting complex stimuli (e.g. faces, objects and buildings) either in isolation or as pairs. A weighted average and a weighted sum model were tested by comparing the condition where subjects attended to both items vs. when they attended only to one of them. The results supported the weighted average model suggesting that the two presented stimuli compete for representation. The study also demonstrated that the amount of competition in FFA was limited in case a face was paired with a stimulus belonging to a different category (e.g. building and objects), indicating differences in the inhibitory influence within and between categories.

Jacques and Rossion $(2004,2006)$ investigated context effects in face selection using electroencephalography (EEG). They tested whether the face-related N170 component was sensitive to the context in which a central face (target face) was embedded. The target face was presented with other non-face stimuli (scrambled faces) or together with other faces. The authors observed that the N170 amplitude decreased when two faces were simultaneously presented. In line with the study by Reddy et al. (2009), this result indicates that when a face is paired with a non-face stimulus neural competition between these two stimuli does not occur. It also suggests that two simultaneously presented faces may compete for representation.

In an fMRI study we further investigated this idea by focusing on the interaction between two faces when they were simultaneously presented (Gentile F and BM Jansma, 2010). In particular we tested whether the amount of competition is related to the characteristics of the neural population codes. This hypothesis was inspired by recent studies by Jiang et al. (2006) and Loffler et al. (2005) which suggested that similar faces are more likely to be encoded by the same neural population than dissimilar faces. Therefore, presenting a pair of similar faces would result in a higher competition effect due to the stronger inhibitory connections within the same population. This would lead to a lower fMRI signal within FFA compared to processing dissimilar faces. This working hypothesis was confirmed by our results as we observed a significant decrease in $\mathrm{FMRI}$ signal for the ignore-similar-faces condition vs. the ignore-dissimilar-faces condition in OFA and in FFA. This similarity modulation within the ignore-faces condition suggested that faces were both encoded and competed for representation both in OFA and in FFA. In order to investigate the effect of attention on the 
competitive interactions between faces, we included an attend-to-face condition where the participants had to attend to one of the faces and perform a match to sample task. We observed that during the attend-to-face condition activation in OFA and FFA was indistinguishable for similar and dissimilar faces displays, confirming that attention is needed to bias neural competition in favor of the processing of the target. Overall, these results suggested that face selection takes place via biased competition.

However, due to the sluggishness of the hemodynamic response, it was not possible to track the dynamics of such a mechanism. In the present study we used EEG to investigate when the two main components of BC, "competition" and "bias", take place. The stimuli display, the experimental design and the procedure were identical for the EEG and the fMRI study. The EEG signal was recorded from 32 electrodes. The EEG data were analyzed with Independent Component Analysis (ICA). This type of analysis is relevant especially when several neural mechanisms as perception, neural competition and attention might work in parallel or in overlapping time-windows. In fact, the basic idea of ICA applied to EEG is that over a sufficient amount of time, the brain sources generating the signal on the scalp become temporally independent from each other. In the present case, the EEG dataset was decomposed in 32 time-courses (independent components - ICs). In addition, each IC was associated with a topographic map representing the projection on the scalp of its neural generator. Therefore, the event related potential (ERP) analysis we performed here was not related to the EEG signal at a specific electrode or group of electrodes but to an IC component in correspondence to a specific spatial source.

Several ERP studies showed that the visual processing of a face occurs through two stages: an early and a late stage. The former was first identified by a significant difference for a negative potential around $170 \mathrm{~ms}$ after stimulus onset when faces were compared with non-face stimuli. This component known as the N170 is most prominent at occipitotemporal electrodes (Bentin S, Allison, T., Puce, A., Perez, E., and McCarthy, G., 1996; Rossion B et al., 1999). The N170 has been shown to be responsible for the featural encoding of a face (Eimer M, 2000). The first ERP component sensitive to the holistic representation of a face is the occipitotemporal negativity N250 (Nasr S and H Esteky, 2009).

Based on the results of the preceding fMRI study (Gentile F and BM Jansma, 2010), we expected that the two faces were automatically encoded and competed for representation when they were ignored. In addition, the ERP/ICs analysis allowed us to separately focus on the N170 and the N250 and to investigate at which stage of face processing neural competition takes place. Therefore, we expected to find a similarity modulation in the ignore-faces condition (IGN_SIM<IGN_DISS) either at the level of the N170 or in correspondence of the N250.

In the $\mathrm{fMRI}$ experiment, the similarity factor modulated OFA and FFA only within the ignore-faces condition. The fMRI signal was comparable in the attend-to-face task for similar and dissimilar faces (ATT_SIM=ATT_DISS). This lack of similarity modulation suggested that attending to one of the two faces solved (or biased) neural competition towards the process- 
ing of that face only. In line with this result we expected to observe the same bias effect at that stage of face processing sensitive to the mutual interaction between the two faces.

OFA and FFA (as measured in FMRI) also showed a main effect of attention (ATT>IGN). A large network of frontal regions was detected as a potential source of this attentional modulation and in turn of the bias signal that solved neural competition between the two faces. In the present study the high temporal resolution of EEG was used to verify this hypothesis. We investigated whether the two mechanisms (attention and bias) have a common origin or whether they are functionally distinct in time and location. Differences in temporal processing of various neural events were identified via latency shifts between occipitally and frontally distributed ERP components. In particular, under the assumption that frontal areas drive the $\mathrm{BC}$ process in OFA and FFA, we expected to find an early frontal ERP modulation related to covert attention and selection and a relatively late $B C$ modulation of a facerelated ERP component.

In addition to ERP and ICA component analysis, we performed dipole source modeling on the scalp maps associated with the ICs-time-courses (see above). Compared to the standard approach (Klopp J et al., 2000), the advantage of solving the inverse problem on the ICmaps is that each IC represents the activity of a single cortical source which generates a specific spatial pattern on the scalp (IC-map). In particular, each IC-map was modeled with a single active equivalent dipole whose projection best-fitted the spatial distribution of that map. Therefore, this new approach offered the opportunity to reliably localize the cortical generators of the N170-ICs and the N250-ICs and, in turn, to gain insight into the flow of information between OFA and FFA in the FMRI experiment. In fact, models of face perception suggested that the two face-selective regions might be functionally connected in a bottom-up (Haxby JV et al., 2000) or top-down manner (Rossion B et al., 2003). The source modeling of the ICs allowed us to investigate whether the pattern of activations we observed in FFA was caused by OFA or vice versa.

\section{Methods}

\subsection{Subjects}

Nineteen healthy volunteers (17 females, two left-handed) with normal or corrected to normal visual acuity participated in this study. The age of participants ranged from 18 to 28 years. They were all students recruited at Maastricht University and their participation was compensated with academic credit points. After explanation of the procedures, participants signed an informed consent form. The study was approved by the ethical committee of the faculty. One of the subjects was discarded from the analysis due to technical difficulties during the EEG measurement. 


\subsection{Stimulus preparation}

The stimuli used in the present experiment were the same employed in the previous fMRI study (Chapter 2). In particular, they consisted of two sets of 16 pairs of faces. One set represented similar (SIM) and the other dissimilar (DISS) faces (see Figure 1, 3.a in Chapter 2 for examples). The faces in the pairs were selected from a large set of pictures of faces (total of 60 pictures) provided by Mark Steyvers (http://psiexp.ss.uci.edu/research/software.htm). The pictures were all taken under similar lightning with neutral facial expressions (Kayser $\mathrm{S}$, 1985).

Similarity between faces was defined by using the two major approaches adopted for face representation: the purely psychological or top-down approach and the purely computational or bottom-up approach (see Figure 1.a, b in Chapter 2). Both approaches are based on the multidimensional representation of faces (Steyvers M, \& Busey, T. , 2000) and are described in details in "Stimulus preparation" in Chapter 2.

The final set of stimuli was derived as follows: a pair of faces was labeled as a SIM (or DISS) pair, only if both faces were labeled as such in the top-down as well as in the bottomup approach (see Figure 1.c in Chapter 2). As a last step, we computed the distance of both sets of faces from a mean face in order to avoid differences in the level of activation within FFA, across conditions (Loffler $\mathrm{G}$ et al., 2005). SIM and DISS faces were, on average, at the same distance from the mean face.

\subsection{Procedure in the main experiment}

The experiment consisted of four runs in a block-design (Figure 1). Each run consisted of 4 blocks and started with a blank screen with a white fixation cross lasting for 3 seconds. At the beginning of each block a visual instruction (attend-to-face and ignore-faces condition, see below) was presented for 3 seconds, followed by a white cross on a blank screen after which the trial started. A block consisted of thirty-nine trials. A single trial started with the presentation of a white fixation cross in the centre of a blank screen of varying duration (mean $2500 \mathrm{~ms}$, range $2000-3000 \mathrm{~ms}$ ), followed by a pair of faces presented for $500 \mathrm{~ms}$. The two faces were positioned in opposite hemifields and they were either SIM or DISS to each other. The single pictures subtended approximately $4.1^{\circ}$ (height) $\times 3.5^{\circ}$ (width) of visual angle and were aligned to the centre of the screen. The entire visual display covered $4.1^{\circ}$ (height) $\times 7.8^{\circ}$ (width) of visual angle. The display size was designed to be comparable with those used in previous studies on face processing (Gauthier I et al., 2000; Halgren E et al., 1999; Kanwisher N et al., 1997; Rossion B et al., 2003; Steeves JK et al., 2006; Wojciulik E et al., 1998). Therefore, it was reasonable to assume that FFA integrated the two simultaneously presented faces within each experimental trial (Halgren E et al., 1999; Reinholz J and S Pollmann, 2007). When the two faces were presented, the fixation cross changed into two 
bars $\left(0.2^{\circ}\right.$ in height $)$ that were placed between the two faces $\left(0.17^{\circ}\right.$ above and below the centre of the screen). The width of the bars varied between 1 and 5 pixels. A block of trials ended with a resting condition consisting of a blank screen with a white cross lasting for 2 seconds. The instruction signalled the type of task to be performed in the following block: an ignore-faces (IGN) and an attend-to-face task (ATT) (see Figure 3.a and Procedure in the main experiment in Chapter 2, for a detailed description of both tasks). In the ignore-faces and attend-to-face condition, targets occurred on average $20 \%$ of the time. The subject was asked to respond as fast and accurately as possible within a $1500 \mathrm{~ms}$ time-window (comprising the face pair display duration and 1 second of blank screen).

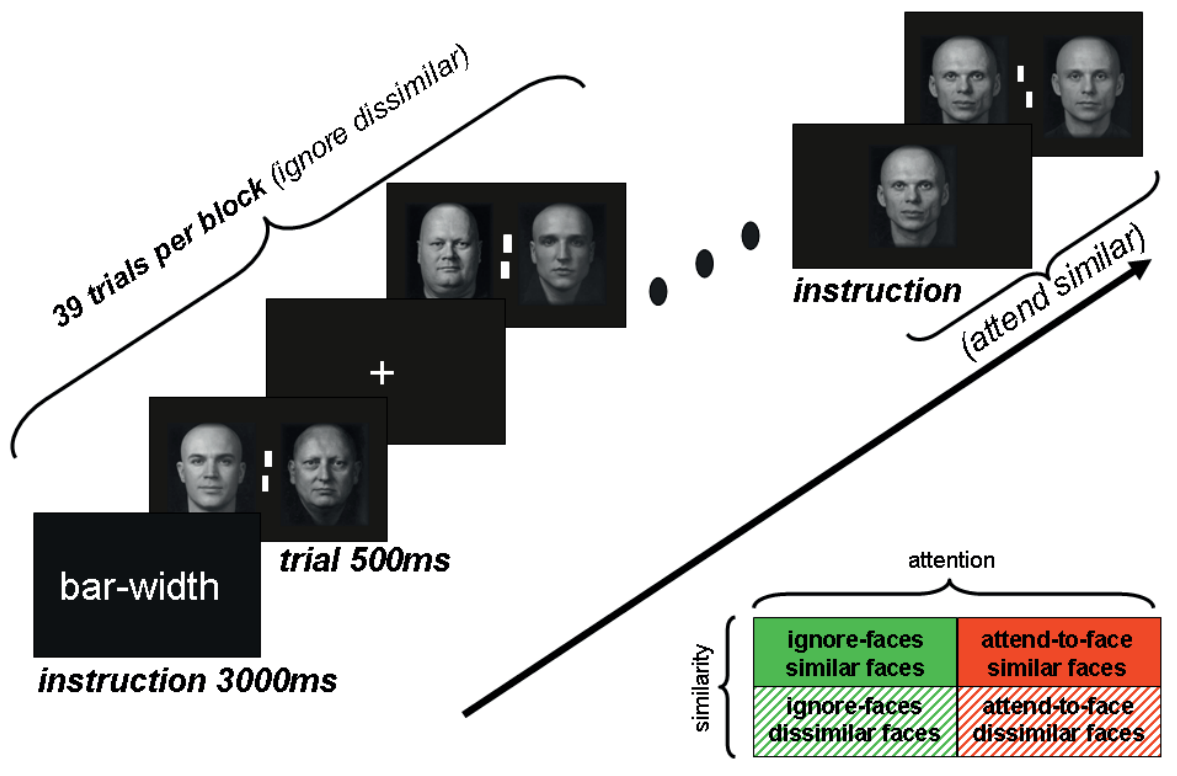

Figure 1. Experimental design. (a) Block design with examples of two different conditions (ignore-faces with dissimilar display and attend-to-face with similar display).

The design was a $2 \times 2$ repeated measures design with factors task (ATT, IGN) and similarity (SIM, DISS), leading to 4 conditions (IGN_DISS, IGN_SIM, ATT_SIM, ATT_DISS, see Figure 3.b in Chapter 2). The participants were not made aware of the similarity manipulation between the two faces. The order of the blocks was randomised and counterbalanced across participants and runs by means of the Williams design (Williams EJ, 1949). This scheme is a generalized Latin square design, balanced for first-order carryover effects. The stimulus display was identical for both types of task. After the explanation of the task, and application of the electrodes, participants were comfortably seated in an electrically shielded, soundattenuated room in front of a computer monitor at a distance of 80 centimeters. The stimuli 
were delivered using the Presentation 12.2 package (Neurobehavioral Systems, Inc. www.neurobs.com). Participants were instructed to fixate the centre of the screen throughout the experiment and to minimise head and eye-movements for the whole duration of the experiment. The entire session lasted approximately 60 minutes.

\subsection{EEG recording}

The electroencephalogram (EEG) was measured using 29 tin electrodes mounted in an elastic cap (QuickCap, Neuroscan Inc. Tm). The electrodes were positioned according to the international 10-20 system (Klem GH et al., 1999) with the electrodes Fz, F3, F4, F7, F8, FCz, FC3, FC4, FP1, FP2, AFz, T3, T4, T5, T6, TP7, TP8, Cz, C3, C4, CPz, CP3, CP4, Pz, P3, P4, Oz, O1, O2. Reference electrodes were positioned at both mastoids (A1, A2). The left mastoid (A1) was used as online reference for the electrical signal measured on the scalp. Vertical eye movements and eye-blinks were monitored by a bipolar montage placed above and below the left upper and lower orbital ridge. Horizontal eye movements were recorded with a bipolar montage placed at the left and right canthi. Electrode impedance was kept below 5 $\mathrm{K} \Omega$. EEG data were recorded and amplified using Neuroscan SynAmps with a $0.05-30 \mathrm{~Hz}$ bandpass and sampled at 4-ms intervals $(250 \mathrm{~Hz})$.

\subsection{Analysis}

EEG data were epoched with an interval of $900 \mathrm{~ms}$, time-locked to the onset of the presentation of the pair of faces. Epochs included a 100-ms pre-stimulus baseline correction interval and they were $0.3-30 \mathrm{~Hz}$ bandpass filtered (Butterworth, zero phase, 24dB/oct).

Data were decomposed in independent components (ICS) via ICA (Onton J and S Makeig, 2006). The ICA algorithm takes as input the EEG signal recorded at the scalp channels and considers each of these signals as a linear mixture of temporal independent components. ICA searches for those time courses in the mixture that are maximally independent to each other. The ICs represent the brain sources that give rise to the signal recorded over the scalp. This assumption arises from the observation that the density of local synaptic contacts among pyramidal cells is much larger than the long-range connections. Therefore, it is plausible to consider, across a reasonable amount of time, the brain as divided into separate functional areas. These areas are supposed to contribute to temporally independent neural processes within a time-window of interest. The ICA model also assumes that these activities originating in the cortex are spatially constant and project onto the scalp instantaneously and linearly via volume conduction. As brain sources are not the only sources contributing to the signal on the scalp, different type of artifacts (e.g. eye-blinks, muscle and heart beat artifact) are also included in the model. In summary, the signal at each electrode can be considered as a linear mixture of independent cortical and non-cortical sources that is spatially fixed over time and that are identified by ICA. 
Before running the ICA we removed those artifacts that were particularly problematic for the ICA algorithm. They mainly consisted of large muscle movements that give rise to high amplitude and high frequency noise. In addition, large muscle movements and other irregular artifacts are unique, non-stereotyped and most importantly associated with one single component each. Visual inspection was used to remove the epochs characterized by this type of artifacts.

After the pruning procedure, the concatenated single-trial data sets were decomposed using infomax ICA implemented in EEGLAB (http://sccn.ucsd.edu/eeglab). The ICA decomposition gave rise to 32 temporal ICS (complete decomposition) and for each component 32 weights were extracted. These weights represented the relative contribution of a specific component to the 32 electrode signal. Therefore, a single IC was characterized by a time course and a scalp map made of the weights associated to that component. From the IC time course were extracted the ERPs related to the 4 conditions (IGN_SIM, IGN_DISS, ATT_SIM, ATT_DISS) excluding target trials. The scalp topography and the event-related average of the IC time course were used to separate task-related and artifacts components. We isolated stereotyped artifact, repeating over time, like eye-movement, eye-blink and some restricted category of muscle movement (e.g. heart beat and muscle tension). The remaining brainactivity related components were collected from all subjects.

We also modeled the ICs scalp maps with one or two equivalent current dipole models by using the DIPFIT algorithm of EEGLAB. The components fitting the model with a residual variance lower than $15 \%$ were kept for further analysis. These components were, in turn, divided into clusters according to their scalp distribution and temporal dynamics. We first defined a few groups of components characterized by the same spatial distribution of their scalp maps. Similarly, we defined groups of ICs identified by an ERP time course that was represented by a prominent negativity or positivity in a specific time-window. The ICs that had the same ERP morphology and spatial distribution (scalp maps) were clustered across subjects.

Statistics on the components in a cluster were performed on the associated ERPs. In particular, we computed for each ERP the peak latency of the negativity (or positivity) characterizing that cluster. The mean amplitude was calculated for a time-window +/- $30 \mathrm{~ms}$ around the peak for all conditions, subjects, and components. In order to have one single statistical entry per subject, the corresponding amplitude values were averaged together if several ICs were associated to the same subjects. These values were submitted to a repeated measures ANOVA. The same statistical analysis as for the IC-ERPs was performed by considering the minimum (maximum) envelope of their back-projections on the scalp. The backprojections of the ICs were then used to evaluate the contribution of each cluster to the variance related to the grand ERP average in a specific time-window. 


\section{Results}

\subsection{Behavioral Results}

We computed error rates (ERs) and reaction times (RTS) on the IGN and ATT task for SIM and DISS conditions. Mean error rates and RTs were then submitted to a repeated measures ANOVA with two factors attention and similarity. No significant main effects of attention ( $F$ $(1,17)=0.01, p=.934$, mean RT ATT $=738 \mathrm{~ms}, \mathrm{SE}=29, \mathrm{IGN}=737 \mathrm{~ms}, \mathrm{SE}=36)$, similarity $(\mathrm{F}$ $(1,17)=1.08, p=.313$, mean RT SIM $=730 \mathrm{~ms}, \mathrm{SE}=28$, DISS $=746 \mathrm{~ms}, \mathrm{SE}=36)$ or interaction $(F(1,17)=0.25, p=.620)$ were observed for RTs. The same analysis on ERs did not reveal any main effect of attention $(F(1,17)=0.00, p=.953$, mean ER ATT $=28 \%, S E=5, I G N=28 \%$, $\mathrm{SE}=3)$ or similarity $(\mathrm{F}(1,17)=1.65, \mathrm{p}=.216$, mean $\mathrm{ER} \mathrm{SIM}=26 \%, \mathrm{SE}=2, \mathrm{DISS}=30 \%$, SE $=$ 5). Unlike the RTs results, the interaction of factors attention and similarity was significant for the ERs $(F(1,17)=6.75, p=.019)$. The pairwise comparisons showed that the difference between dissimilar and similar condition within the attend-to-faces condition approached significance (ATT_SIM < ATT_DISS: $F=3.59, p=.075 ;$ IGN_SIM $>$ IGN_SIM: $F=2.23, p=0.153$ ).

\subsection{ICA clusters and estimated sources}

The ICA cluster analysis revealed 3 clusters of interest namely the N170, N250 and N2bcluster. The N170-cluster consisted of 11 components from 11 subjects. They were characterized by a negativity peaking around $180 \mathrm{~ms}$. A $2 \times 2$ ANOVA performed on the ICs did not reveal any main effect of attention, similarity or interaction of factors for this cluster (Figure 2.a).

The IC scalp maps showed a central occipital distribution. These maps were used for source dipole modeling. In particular, we assumed that the N170 was generated by two bilateral cortical sources. In fact, the ICA extracts one central component from two synchronized sources. Moreover, the N170 has been associated with the first phase of face encoding corresponding anatomically with a face selective region located on the lateral bank of the lateral occipital complex (LOC) and defined as OFA. Therefore, in order to investigate whether OFA was a realistic source of the N170, we seeded a two-equivalent current dipole into the centro-lateral part of the inferior occipital gyrus (Figure 2.b). This source dipole model accounted on average for $98.41 \%$ of the observed variance $(97.29 \%-99.15 \%)$. 

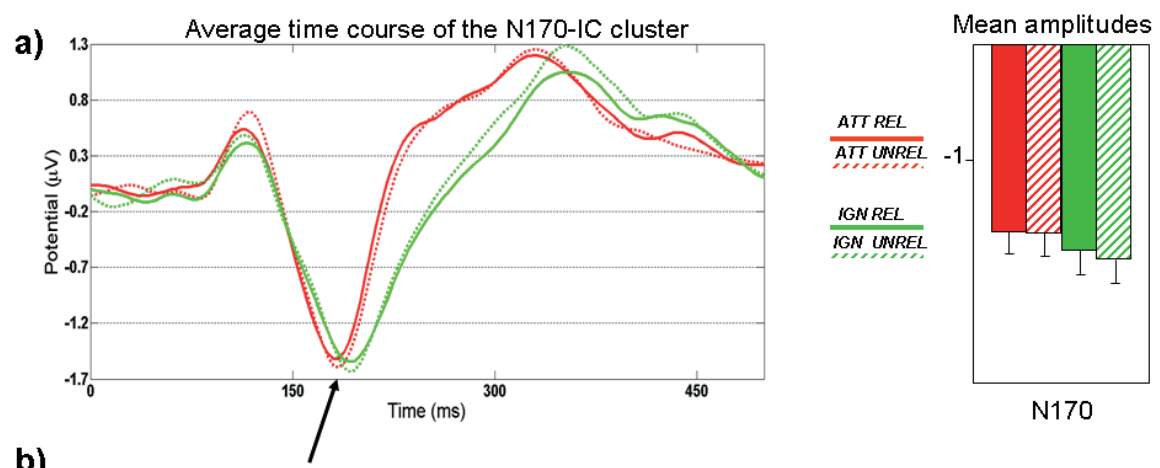

b)

ICs contribution over the scalp

average distribution
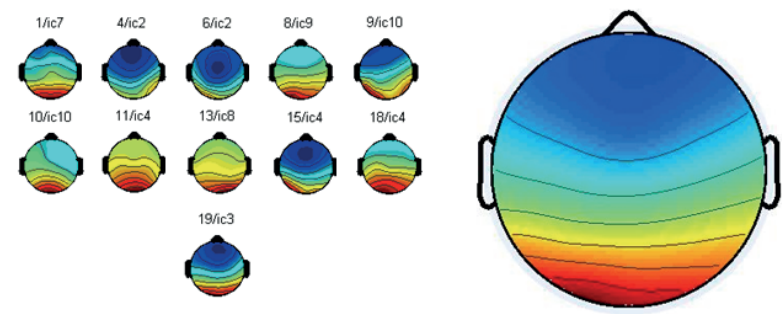

dipole source modeling

$98 \%$

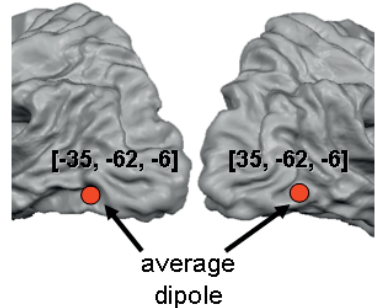

Figure 2. Result of the ICA analysis of the EEG data: the N170. (a) Left: Average of the ERPs associated with the ICs of the N170-cluster. Right: results of the 2×2 ANOVA analysis on the mean-ERP-amplitudes of the ICs in a window of 60 $\mathrm{ms}$ around $180 \mathrm{~ms}$ (average peak). (b) Left: scalp maps associated to the ICs of the N170-cluster. Center: Average of the ICs spatial distribution. Right: location of the two-dipoles-model used to fit the ICs scalp maps.

The clustering procedure revealed another face-specific cluster defined as the N250-cluster whose components (17 ICs extracted from 12 subjects) were identified by a negative deflection around $230 \mathrm{~ms}$. The statistical analysis on these components revealed a significant interaction of factors attention and similarity (Figure 3.a). Attention: $(F(1,11)=.017, p=$ .898 , mean amplitude ATT $=-1.4, \mathrm{SE}=.423, \mathrm{IGN}=-1.4, \mathrm{SE}=.395)$. Similarity: $(\mathrm{F}(1,11)=$ $1.896, \mathrm{p}=.196$, mean amplitude $\mathrm{SIM}=-1.4, \mathrm{SE}=.409$, DISS $=-1.46, \mathrm{SE}=.399)$. Interaction: $(\mathrm{F}$ $(1,11)=5.316, p=.042)$. The interaction effect was further investigated by comparing the similar vs. dissimilar condition within the attend-to-face and the ignore-faces condition separately. The pairwise comparisons showed no difference in the attend-to-face condition, but a significant effect of similarity within the ignore-faces condition (ATT_SIM > ATT_DISS: $F$ $=.10, p=.756$; IGN_SIM < IGN_DISS: $F=8.67 \mathrm{p}=.013$ ). The same $2 \times 2$ ANOVA was performed on the back-projections of the ICs onto the scalp (original space). This analysis, in line with the previous one, revealed an interaction effect $(F(1,11)=8.46, p=.014)$. This interaction reflected, once again, no similarity effect within the attend-to-face condition but a signifi- 
cant difference between the ignore-similar-faces and ignore-dissimilar faces conditions (ATT_SIM > ATT_DISS: $F=.17, p=.688$; IGN_SIM <. IGN_DISS: $F=7.59, p=.019$ ).

The IC scalp maps were characterized by a posterior-central distribution. Several studies suggested, based on source modeling, that the N250 is generated in FFA (Schweinberger SR et al., 2004; Schweinberger SR et al., 2007; Schweinberger SR et al., 2002). In addition, our fMRI study showed an interaction effect between attention and similarity bilaterally within FFA. Therefore, similarly to the seeding procedure used for the N170, we modeled the scalp maps of the N250-cluster with two symmetrical dipoles located bilaterally in the anterior part of fusiform gyrus (Figure 3.b). These dipole models located within FFA accounted on average for $93.22 \%$ of the total variance (90.17\% - $97.47 \%)$.
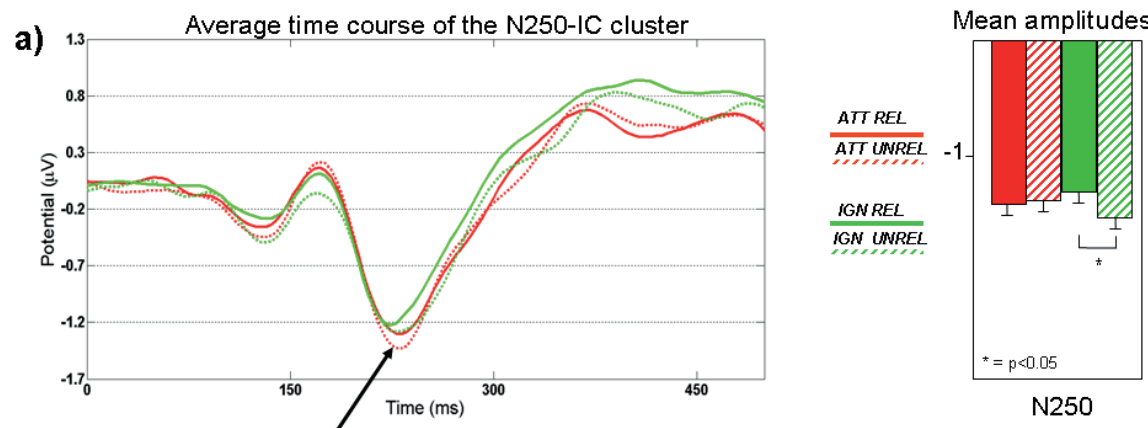

b)
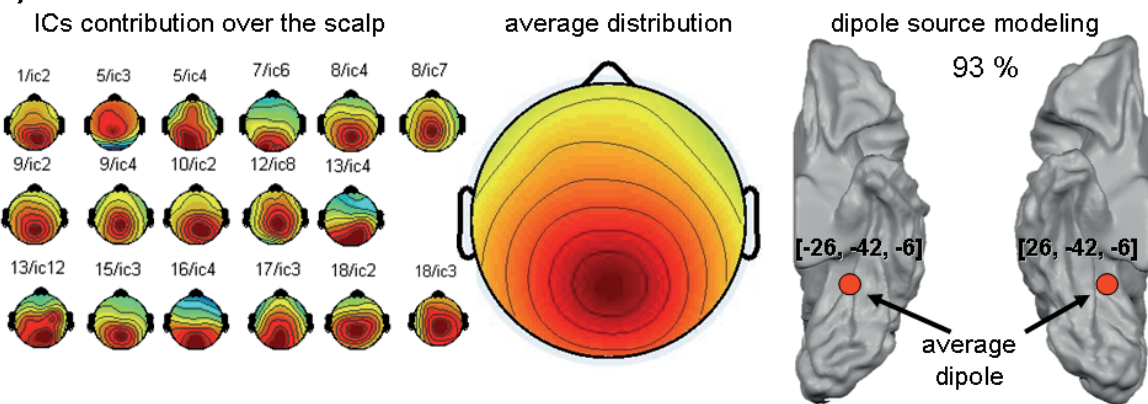

Figure 3. Result of the ICA analysis of the EEG data: the N250. (a) Left: Average of the ERPs associated with the ICS of the N250-cluster. Right: results of the 2×2 ANOVA analysis on the mean-ERP-amplitudes of the ICs in a window of 60 $\mathrm{ms}$ around $230 \mathrm{~ms}$ (average peak). (b) Left: scalp maps associated to the ICs of the N250-cluster. Center: Average of the ICs spatial distribution. Right: location of the two-dipoles-model used to fit the ICs scalp maps.

The N2b-cluster consisted of 15 components from 11 subjects. The ICs in this cluster were characterized by a large negativity around $270 \mathrm{~ms}$. A $2 \times 2$ ANOVA on the ICs mean amplitudes revealed a main effect of attention and no main effect of similarity or interaction (Figure 4.a). Attention: $(F(1,10)=45.092, p=.000$, mean amplitude $\mathrm{ATT}=-1.649, \mathrm{SE}=.327, \mathrm{IGN}=-$ $.545, \mathrm{SE}=.226)$. Similarity: $(\mathrm{F}(1,10)=.001, \mathrm{p}=.980$, mean amplitude $\mathrm{SIM}=-1.096, \mathrm{SE}=$ .297 , DISS $=-1.099, \mathrm{SE}=.250)$. Interaction: $(F(1,10)=.312, p=.589)$. We found the same 
pattern of results when we back-projected the ICs of the N2b-cluster onto the scalp. In fact, a $2 \times 2$ ANOVA revealed a main effect of attention $(F(1,10)=29.427, p=.000$, mean amplitude $\mathrm{ATT}=-3.065, \mathrm{SE}=.595, \mathrm{IGN}=-1.324, \mathrm{SE}=.325)$ and no other effects.

a)

b)

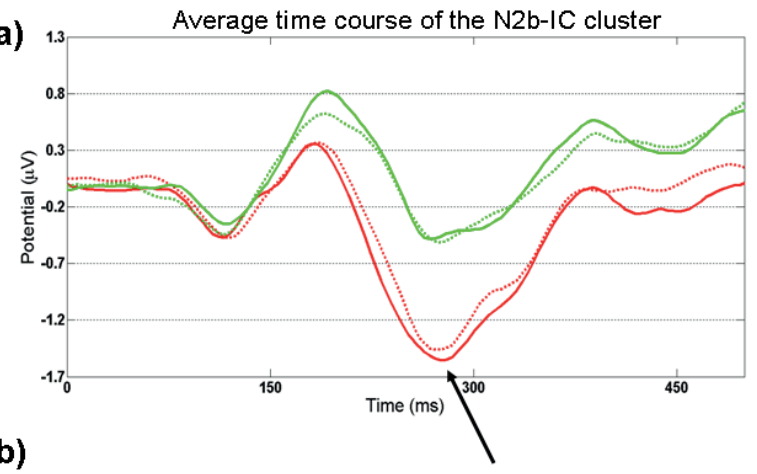

ICs contribution over the scalp

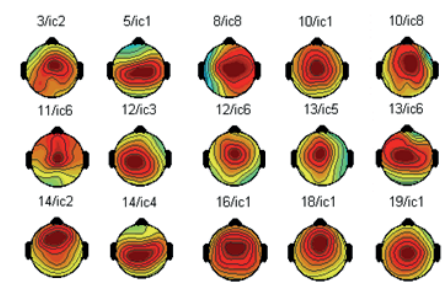

average distribution

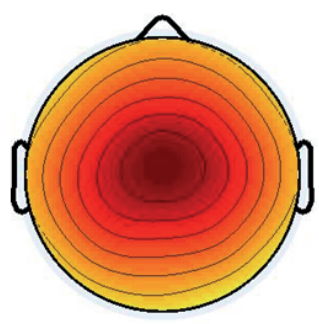

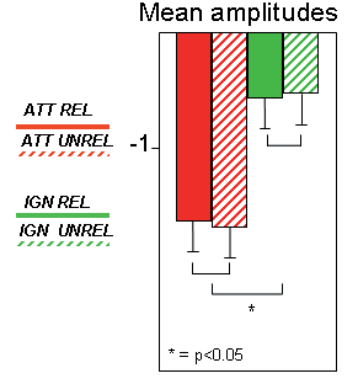

$\mathrm{N} 2 \mathrm{~b}$

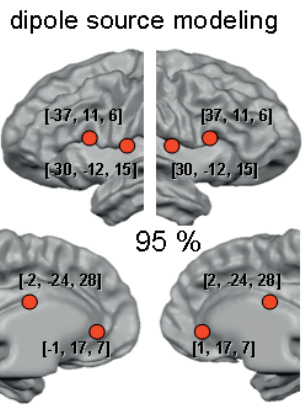

Figure 4. Result of the ICA analysis of the EEG data: N2b. (a) Left: Average of the ERPs associated with the ICs of the N2b-cluster. Right: results of the 2×2 ANOVA analysis on the mean-ERP-amplitudes of the ICs in a window of $60 \mathrm{~ms}$ around $270 \mathrm{~ms}$. (b) Left: scalp maps associated to the ICs of the N2b-cluster. Center: Average of the ICs spatial distribution. Right: location of the dipoles-models used to fit the ICs scalp maps.

The topography associated with the ICs in the N2b-cluster had a fronto-central scalp distribution. In contrast to the N170 and N250 analysis, for the N2b we did not have a clear expectation on the source location of the dipole models. In fact based on the fMRI results several frontal areas (bilaterally within the insula and in the right hemisphere within the inferior frontal gyrus, precentral gyrus/sulcus) were involved in the attentional task. Therefore, we did not proceed with seeding dipoles in a specific area. Instead, we computed for each IC an equivalent current dipole (a one or two dipole model) characterized by a projection on the scalp that best-fitted the observed scalp topography of that component. The best fitting dipoles mainly corresponded to a network of regions in the prefrontal cortex comprising the cingulate gyrus, the insula and the inferior frontal gyrus (IFG) (Figure 4.b). On average these dipoles accounted for $95.04 \%$ of the total variance ( $89.21 \%$ - $99.10 \%)$.

In order to test whether the three described clusters were representative respectively for the N170, N250 and N2b ERP component on the scalp, we computed the contribution of 
the IC-back-projections to the grand average ERP in three corresponding time-windows: 140 - $220 \mathrm{~ms}$ (N170), 170-250 ms (N250) and 220 - $300 \mathrm{~ms}$ (N2b). In the 140 - $220 \mathrm{~ms}$ timewindow the ICs in the N170-cluster were the components that contributed the most to the grand average for all 4 conditions (ATT_SIM $=38.55 \%$, ATT_DISS $=40.88 \%$, IGN_SIM $=48.34$ $\%$, IGN_DISS $=55.11 \%$ ). In the second $170-250$ ms time-window we observed that the N250 was the cluster that contributed the most to the signal on the scalp in the ignore-faces conditions (IGN_SIM $=27.59 \%$, IGN_DISS = $28.28 \%$ ). In the same time-window both the N250 and $\mathrm{N} 2 \mathrm{~b}$-clusters were relevant in explaining the overall signal in the attend-to-face condition (N250: ATT_SIM $=31.15 \%$, ATT_DISS $=33.37 \%$; N2b: ATT_SIM $=34.35 \%$, ATT_DISS = $37.39 \%)$. In the third $220-300$ ms time-window the N2b-cluster was the one that contributed the most but only in the attend-to-face condition (ATT_SIM $=23.57 \%$, ATT_DISS = $27.36 \%)$.

\section{Discussion}

In the present study we investigated the temporal properties of face selection in the context of $B C$, using EEG and ICA. The BC model states that neural representations of simultaneously presented items compete with each other. Attention biases the neural competition in favor of the object relevant for further cognitive processes and behavior. In a preceding $\mathrm{fMRI}$ study (Gentile F and BM Jansma, 2010) we tested the BC model by using the same face stimuli, procedure and design as described here. We observed both in OFA and FFA a BC-like activation pattern (IGN_SIM<IGN_DISS - competition; ATT_SIM=ATT_DISS - bias). In addition, frontal areas, such as insula, inferior frontal gyrus and precentral gyrus/sulcus showed a main effect of attention and no similarity effect (ATT>IGN), suggesting that these areas triggered the bias (selection) downstream towards OFA and FFA (Gentile F and BM Jansma, 2010). However, the signal we observed in the fMRI experiment was a "summary" of neural events occurring during $500 \mathrm{~ms}$ (trial duration), and it could not be used to study temporal dynamics. In the present study we used the high temporal resolution of EEG and focused on ERP components related to face processing and selection. We tested which of these ERP components were sensitive to neural interactions between competitive items (faces) and to the attentional signal that eventually biased such interactions towards the relevant face. The ERP analysis was also used to investigate whether the origin of the attentional modulation that biased competition in OFA and FFA was caused by top-down signals from frontal areas as suggested by our fMRI results.

In this experiment subjects had to carry out a complex task. Several neural mechanisms like face processing (i.e., encoding of facial features, the holistic configuration of a face and face recognition), attention (attentional shift and bias) and response execution were involved at the same time. This means that several neural sources were at work simultaneously. As a consequence, several ERP components related to these functions temporally and 
spatially overlapped on the scalp. In order to investigate the access of the visual information of both face as well as the selection of the relevant one within each source, we needed to disentangle these processes into independent events. This was accomplished by analyzing the EEG data using ICA. The efficacy of this tool has been shown in several EEG experiments both in the temporal (Debener S, S Makeig et al., 2005; Debener S, M Ullsperger et al., 2005; Makeig S, M Westerfield, TP Jung et al., 1999; Makeig S, M Westerfield, J Townsend et al., 1999) and spectral (Makeig S et al., 2004; Makeig S et al., 2002; Onton J et al., 2005; Riecke L et al., 2009) domain. We used ICA to search for ICs that represented the ERPs related to the processes involved in the two tasks (ignore-faces/attend-to-face tasks) for the two types of stimuli (similar/dissimilar faces).

The ICs extracted for all subjects were divided into clusters according to their temporal and spatial characteristics. In line with the cognitive processes related to the subjects' task we found ICs-clusters related to ERP components indexing face processing and spatial attention namely the N170, N250 and the N2b. In a nutshell, the pattern of result at the N170 (no effects) suggested that no competition or bias effect takes place at an early phase of face processing. The analysis at the level of the N250 indicated that faces compete for representation in the ignore-faces condition in a later time-window where the holistic encoding of a face occurred. In the same time-window, we found that attention biased neural competition towards the attended face. However, we did not observe a main effect of attention in the N250 time-window. The lack of an attentional effect for the N250 differentiates this ERP component from the pattern of FMRI signal we observed in OFA and FFA. This difference between the $\mathrm{fMRI}$ results in face selective areas and the N250 suggested that the attentional effect in the fMRI data could only arise from later top-down processing. It also indicated that the significant difference between attend-to-face and ignored-faces condition within OFA and FFA was an effect of temporal integration of the fMRI signal related to several cognitive processes over time. In fact, the main effect of attention we found in the $\mathrm{N} 2 \mathrm{~b}$ confirmed that the enhancement of activation in OFA and FFA in the attend-to-face condition might be due to an attentional and/or task dependent top-down effect. The observation that the N2b evolves around $50 \mathrm{~ms}$ later than the bias effect in the N250 suggested that bias and topdown control are independent from each other, and that bias precedes top-down taskrelated attentional modulation. 


\subsection{The N170-cluster}

The IC cluster analysis clearly identified a N170-cluster whose components were characterized by a negative deflection around $180 \mathrm{~ms}$. In order to validate the "goodness" of this cluster as a representative of the N170 ERP on the scalp we computed the variance explained by the back-projected ICs to the negative signal on the scalp. The N170-cluster turned out to be the one that contributed the most to the signal on the scalp in the $140-$ 220 ms time-window. The topography associated with the ICs showed an occipito-central distribution. The N170 has been functionally and anatomically associated with OFA, more specifically with the early phase of face perception consisting with the structural encoding of a face (Eimer M, 2000; Itier RJ and MJ Taylor, 2002). This correspondence was confirmed by modeling the IC-scalp maps with two equivalent dipoles located in the lateral bank of LOC, recently identified with OFA ( $96.23 \%$ averaged explained variance).

Although the N170 and the OFA (as measured in fMRI) showed a reasonable anatomical fit, the two measurements (hemodynamic imaging and electrophysiological recording) differed in several aspects at the functional level. The statistical analysis on the mean amplitude around the N170 peaks did not show the same interaction and the main effect of attention we observed in the fMRI signal in OFA and FFA. In Gentile F and BM Jansma (2010), we interpreted this pattern of activation (IGN_SIM<IGN_DISS; ATT_SIM=ATT_DISS) as evidence for competitive interactions between the two faces when they were both ignored. This result also showed the effect of the attentional bias that solved the competition towards the face attended to. In contrast, the lack of effects in the N170 suggests that the system has not yet processed the two faces. Alternatively, at this early stage of face processing faces might still be processed in terms of their basic components. Therefore, the absence of any similarity modulation within the ignore-faces condition would imply that faces do not compete at the featural level.

In more general terms, we can conclude that selection of a face in the context of another face does not occur in the N170-time-window and most importantly not in line with the $\mathrm{BC}$ model. In addition, the anatomical correspondence between the N170 and OFA forced us to reconsider the role of OFA in the context of $B C$ and face selection (see below).

Another difference between the EEG and $\mathrm{FMRI}$ results was the lack of any main effect of attention in the N170 that we clearly found at the level of the hemodynamic response in OFA and FFA. In a recent study, Furey ML et al., (2006) showed a very similar divergence while measuring with magnetoencephalography (MEG) and $\mathrm{FMRI}$ the effect of attention on face processing. They observed that the hemodynamic response in the fusiform and inferior occipital cortex was strongly modulated when attention was focused on faces compared to houses. The same pattern was not found at the level of the early face-selective response (M170). The only modulation of the MEG signal was found in a later stage of processing identified as "category-related" component. 


\subsection{The N250-cluster}

The N250-cluster consisted of ICs with a large negativity around $230 \mathrm{~ms}$. IC scalp maps were characterized by a posterior-central distribution. Dipole source analysis suggested that FFA is a plausible source location for the ICs in this cluster (93.22\% averaged explained variance).

The anatomical correspondence between the N250-ICs and FFA (as measured with fMRI) was accompanied by time course similarities of post synaptic (ERP) and hemodynamic response ( $\mathrm{fMRI}$ signal). Both dependent variables showed similar $B C$-like interactions (IGN_SIM<IGN_DISS; ATT_SIM=ATT_DISS). As in FFA, a planned pairwise comparison for the N250-ICs showed a significant similarity modulation in the ignore-faces condition (IGN_SIM<IGN_DISS) and no similarity effect for the attend-to-face condition. The same interaction effect and the same significant difference within the ignore-faces conditions were found for the N250-ICs back-projected on the scalp. In addition, the N250-cluster (together with the $\mathrm{N} 2 \mathrm{~b}$-cluster) contributed the most to the grand average ERP in the timewindow $170-250 \mathrm{~ms}$ for all four conditions. This confirmed the relevance of the N250cluster as representative of the N250 ERP component.

The occipito-temporal N250 has been shown to be face-selective (Nasr S and H Esteky, 2009; Schweinberger SR et al., 2004) and to be responsible for the global configuration of a face. Several studies demonstrated that the N250 is sensitive to face familiarity, face repetition (Pfutze EM et al., 2002; Schweinberger SR et al., 2004; Tanaka JW et al., 2006) and it is thought to be related to face identification. Therefore, it is plausible to assume that the significant difference between similar and dissimilar pairs of faces within the ignore-faces condition reflects a simultaneous encoding of both faces at the holistic level of representation. The direction of the similarity modulation within the ignore-faces condition (IGN_SIM<IGN_DISS) is in line with the neural representation of faces in the context of neural competition. In fact, two recent fMRI studies showed that similar faces are likely to be encoded by the same neural population. In contrast, dissimilar faces are processed by distinct subpopulation of neurons (Jiang X et al., 2006; Loffler $G$ et al., 2005). According to BC, two similar faces would compete more for representation due to larger inhibition among the neurons in the cohort. This would lead to a lower overall activity and signal amplitudes for the processing of similar compared to dissimilar faces. The significant difference between the ignore-dissimilar-faces vs. ignore-similar-faces condition could not be explained by any repetition suppression effect (neural adaptation over time) and its difference between the similar and dissimilar conditions. We ruled out such a possible confound as the similarity between faces was only manipulated within a trial (in a pair) and was kept constant for both conditions across trials.

In line with $\mathrm{BC}$, the lack of similarity modulation within the attend-to-face condition suggested that neural processing was biased towards the perception of the target face only. In fact the N250 might reflect the effect of covert attention to select the relevant face at the target position (Eimer M and M Kiss ; Theeuwes J). The bias effect of attention in a competi- 
tive system was explained by Reynolds and Desimone (1999) by using the "shrinking receptive field" metaphor. In fact, attention to the target face seems to increase the effective spatial resolution of the visual system in order to process only the target in a pair, making it context-independent. This "shrinking" of the receptive field or context independence can be seen as an efficient selection mechanism for relevance in complex scenes - or as tested here - in multiple faces displays.

Therefore, as commented above, the interaction of factors attention and similarity at the level of the N250 suggests that attention only affect a later stage of face processing (Furey ML et al., 2006). However, unlike the fMRI pattern in OFA and FFA, the N250 did not show any main effect of attention. This result is not surprising in the context of BC. In fact, according to the BC model as tested in electrophysiology, two stimuli simultaneously presented and ignored elicited a neural response equivalent to the weighted average of the response to each item alone. In the present study, the stimuli in the pair (faces) were selected in such a way they would elicit the same hemodynamic response in FFA (see Stimulus preparation). Therefore, it is very likely that the weighted average responses to the two faces (ignore-faces condition) matched the response to one single face (attend-to-face condition). The observed attentional modulation in OFA and FFA can only be interpreted as a result of a different process, i.e. a later top-down mechanism (see below). We suggested that this temporal segregation was camouflaged in $\mathrm{fMRI}$ due to the temporal integration of the $\mathrm{fMRI}$ signal across trials and blocks.

In summary, the combination of ICA and ERP analysis revealed that selecting a single face in a pair occurred in a BC-fashion at a relatively late stage of face processing, around 250 ms post-stimulus onset. In Gentile F and BM Jansma (2010), we suggested that this mechanism takes place in OFA and FFA. However, in light with the dipole source modeling for the N170 and N250-ICs, the findings of the present study indicated that FFA plays a central role in selection in the N250-time-window, while the OFA activation pattern might derive from a feedback effect from higher order areas like FFA itself (Rossion B et al., 2003).

\subsection{The N2b-cluster}

In the present study, by using EEG, it was possible to investigate the temporal characteristics and the sources of the attentional effect we observed in OFA and FFA during the FMRI experiment. The ICA analysis revealed a cluster of ICs that we defined as the N2b-cluster based on its spatial and temporal characteristics (200 - 300 ms interval after stimulus onset, distributed frontally and over central electrode sites).

The optimal dipole fits related to the $\mathrm{N} 2 \mathrm{~b}$ activity were located within those frontocentral regions that showed a main effect of attention in the fMRI experiment namely within the insula and the inferior frontal gyrus. In addition, for some ICs the dipoles fitted within the cingulate gyrus. This was in line with several source analyses reporting medial brain 
areas and in particular the anterior cingulate as possible generator of the $\mathrm{N} 2 \mathrm{~b}$ (Lange $\mathrm{JJ}$ et al., 1998).

The N2b-cluster ICs showed a main effect of attention reflecting a significantly larger signal for the attend-to-face than for the ignore-faces condition. The strength of this effect and its relevance over a large time-span was confirmed by the large amount of variance explained by the ICs of the N2b-cluster back-projected on the scalp. In particular, the N2bcluster was the one contributing the most to the signal on the scalp in two relevant timewindows, i.e. in the 170 - $250 \mathrm{~ms}$ and in the 220 - $300 \mathrm{~ms}$ time-windows for the attend-toface condition.

The N2b ERP component is thought to reflect covert orienting attention and the further processing of the selection of the relevant item (Boksem MA et al., 2005; Lange JJ et al., 1998; Luck SJ and SA Hillyard, 1994; Okita T et al., 1985; Sams M et al., 1983; Wijers AA, G Mulder et al., 1989; Wijers AA, L Otten et al., 1989). Lange et al. (1998) also demonstrated that the N2b was elicited for a large set of attended objects confirming its specificity to attentional selection. The N2b was also elicited in match-to-sample tasks where participants were presented with items not matching the actual target (Sams M et al., 1983).

In the attend-to-face condition of the present experiment similar mechanisms were involved. In particular, covert attention took place in order to shift the focus of attention from the center of the screen to the left. Subsequently, selective attention was needed to perform the match-to-sample task. Therefore, the N2b-cluster ICs likely reflected these attentional components. In addition, as in the present analysis all the matched target trials were not included, the N2b-cluster ICs could also be the marker of the template mismatch effect. The effect of attention could not be due to a difference in difficulty between attend-to-face and ignore-faces conditions as behaviorally we did not find any significant difference between the two tasks.

These results confirmed the idea that the attentional main effect in OFA and FFA (fMRI study) originated from a distributed network in the prefrontal cortex, active in the N2b timewindow. They also suggested that this attentional process cannot be the direct cause of the bias effect, as the bias was observed at an earlier latency (at the N250 level). Most likely frontal sources are needed to properly perform the match-to-face task by amplifying the encoding of the "already biased" face.

In conclusion, the present study demonstrated that ICA can be a very powerful tool to investigate the brain dynamics and to (re)interpret the fMRI results. ICA was successfully used here to study the effects of elaborated stimuli such as faces embedded in a complex covert attention task. In fact, the ICA found, in a data-driven fashion, those ERP components known to be the most relevant for face (N170 and N250) and attentional processing (N2b). Most importantly the anatomical and functional correspondence between the $\mathrm{FMRI}$ and ERP/ICs data allowed us to track the temporal profile of the selection mechanism of a face in the context of another face. We showed that when two faces were simultaneously presented 
and ignored they compete for representation in a relatively late stage of face processing (N250) where the global configuration of a face is achieved, in FFA. In addition, when the target face was attended to, similar and dissimilar conditions elicited the same EEG signal. This indicated that in the N250 time-window an attentional bias solved the neural competition between faces within FFA. This interpretation of results supported the $\mathrm{BC}$ model in the context of face selection. Finally, the combination of the EEG and fMRI results suggested that the bias effect that solves neural competition in FFA is not driven by any bottom-up signal from OFA into FFA, neither via top-down modulation from frontal areas, but is FFAspecific and might represent the initial stage of selection.

\section{Acknowledgments}

The authors wish to thank Lisa Jonkman, Peter de Weerd and Mart Bles for helpful discussions. This project was supported by Maastricht University (HRM initiative Mobilizing Minds: "Females into higher positions") to B.M. Jansma. 

Semantic selection in the context of face processing: an fMRI study 


\section{Abstract}

In a social situation, it is very common to meet several people at the same time and to have "small talks" or deeper conversation. In both cases, the degree we know the person we are talking with is an important element for a successful interaction. However, retrieving semantic information about someone we barely know can be very demanding and influenced by the context. In the present fMRI study, we investigated the mechanism underlying the selection of a face associated with a profession that was relevant for the task. More specifically, we presented at the same time two faces that were semantically related or unrelated to each other. The relatedness manipulation was guaranteed by associating each face with a specific profession and by pairing two faces whose professions belonged to the same (related) or different (unrelated) category. The subjects were asked to perform an ignore-faces task, where they had to ignore the faces in the background and perform an unrelated task in the center. We also added an attend-to-face condition, where participants had to attend to the face on the left and perform a match-to-profession task. When the two faces were ignored, we found a relatedness effect in the inferior frontal gyrus (IFG) suggesting that professions from the two faces were simultaneously encoded even when attention was focused somewhere else. This effect was interpreted as reflecting competition of two semantic representations. The lack of relatedness modulation in the attend-to-face condition indicated that attention to the target-face biased this competition towards the semantic processing of the relevant information only. Next to the IFG, also the fusiform face area (FFA) seemed to participate to the process of selection. We observed in this region a relatedness effect within the attend-to-face condition. This result suggested the distracting face was processed in FFA and influenced the integration between the featural and semantic properties of the targetface occurring in this area.

Keywords: semantic selection, face processing, semantic relatedness, fMRI 


\section{Introduction}

When we speak or more in general interact with a person, we often need to retrieve some known background information about him/her in order to afford a proper conversation. This task has to be performed on the basis of the visual characteristics (e.g. eyes color, age) and semantic information (e.g. profession or where he/she lives). In addition, if the conversation occurs in the presence of other people, irrelevant information has to be ignored (e.g. the type of jobs of the peers). In such a situation our cognitive system has to correctly associate and process the visual and semantic information of the target person and filter out the context (non targets) both at the visual and semantic level.

In the visual domain, selection has been largely investigated in a multiple stimuli display. In Chapter 2 and Chapter 3, we investigated via fMRI and EEG the process of face selection in the context of a distracting face (Gentile F and BM Jansma, 2010). In particular we tested whether this mechanism works according to the competitive models of selection proposed for early visual areas (Beck DM and S Kastner, 2005, 2007; Bles M et al., 2006; Kastner S et al., 1998; Kastner S et al., 2001; Kastner S et al., 1999). These models rejected the classical view of attention working as a spotlight that enhances the neural response of the target. They rather stated that whenever two items are simultaneously presented they interact with each other in a mutual suppressive way. In line with the competitive models of selection, several authors observed that attending to one of the stimuli biased the processing towards the relevant item only (Chelazzi L et al., 1998; Chelazzi L et al., 2001; Desimone R and J Duncan, 1995; Luck SJ et al., 1997; Miller EK et al., 1993; Moran J and R Desimone, 1985; Reynolds JH et al., 1999; Treue S and JC Martinez Trujillo, 1999; Treue S and JH Maunsell, 1996). In our experiment, we simultaneously presented faces that were similar or dissimilar at the physical level. We asked participants to perform an attend-to-face condition where they covertly attended to one face (selection phase). In addition, they carried out an ignore-faces task where they ignored both background-faces (competition phase). We observed that the context played a role only within the ignore-faces condition. These results suggested that faces were automatically encoded at the visual level and in turn compete for representation in FFA. In contrast, when the relevant face was to be selected (attend-to-face task) the neural activity in FFA increased regardless of the similarity manipulation between the two faces. This pattern in FFA suggested that attention actually biased neural competition in favor of the processing of the target face. We also found by using EEG that both competitive interactions and bias effect occurred at a relatively early time-window at about 250 ms (see Chapter 3).

A similar model of selection, based on competitive interactions between different representations was proposed also in the semantic domain (Kan IP and SL Thompson-Schill, 2004). This model was tested by comparing visual stimuli that triggered multiple semantic 
representations (e.g a picture of a parrot-like bird can be associated with different name alternatives as, for example, bird, parrot or cockatoo) and items with only one possible name (e.g. a picture of a zebra). The task of the participants consisted in naming the stimuli presented. Naming latency and the IFG activity were positively correlated with the amount of different name-options (Kan IP and SL Thompson-Schill, 2004; Kremin H et al., 2000; Levelt WJM et al., 1991; Thompson-Schill SL et al., 1997; Vigliocco G et al., 2002). These studies supported the idea that when the stimulus is presented, after an initial perceptual analysis and prior to selection, the system generates all possible semantic representations related to that stimulus (Levelt WJ et al., 1998; Levelt WJM et al., 1991). The simultaneous coactivation of those semantic nodes results in competitive interactions, which are stronger with a higher number of activated candidates in the semantic space or lexicon. Kan IP and SL ThompsonSchill (2004) suggested that the neural competition occurring in the IFG was "solved" if the semantic alternatives of a stimulus were biased towards a unique meaning. It is worthwhile to notice that in all these studies the occurrence of semantic competition was indirectly inferred from the task demand related to the selection of a specific meaning. However, from the moment the visual stimulus was presented up to its selection several processes like the perceptual analysis of the item, the simultaneous activation of several word candidates and neural competition among those candidates took place. Finally the actual selection was accomplished when one of the name options was chosen. The occurrence of these different stages makes, in turn, the identification of the competitive mechanism as well as the mechanism more strictly related to selection, very difficult.

The present study aimed to disentangle these two phases (competition and selection) in the context of face processing. Competitive interactions among different semantic representations were tested by simultaneously presenting two faces that had to be ignored (ignorefaces task). We assumed, in this condition, that the semantic information related to the faces was actually encoded though ignored. Several studies using different types of stimuli and paradigms (e.g. negative priming or semantic interference designs), supported this assumption by showing that unattended objects are processed both at the semantic (Di Pace E et al., 1991; Eddy M et al., 2006; Tipper SP and J Driver, 1988; Yee PL, 1991) and phonological level (Bles M and BM Jansma, 2008). In order to vary the amount of competition in a controlled manner we associated the two faces in the pair with the same category professions (semantically related) or with professions from two different categories (unrelated). We kept the perceptual context constant as we always presented two faces that were visually dissimilar to each other (see Stimuli preparation). Therefore any competitive effect due to perceptual differences was to be excluded. The phase of semantic selection was explored by using an attend-to-face task. In this task the subjects had to attend to one of the faces and match it to a predefined target and associated profession.

The region we expected to play a central role in semantic selection of faces was the IFG. This area has been associated to several aspects of semantic processing. In language comprehension the IFG is responsible for the semantic retrieval of a single item and more in 
general for the unification process that integrates over time several types of information during sentence comprehension (phonological, syntactic, and semantic) (Hagoort P, 2005; Price CJ, ; Snijders TM et al., 2009). Moreover, in language production, the IFG was shown to be differentially activated when a single semantic representation had to be selected in a clutter of other options (Grindrod CM et al., 2008; Kan IP and SL Thompson-Schill, 2004; Martin A and LL Chao, 2001). However, as outlined above, none of these production studies was able to separate from each other within the IFG, the mechanisms of lexical access of all the possible names associated with an object, the competition among them and the selection of a single option. In the present design the ignore-faces and the attend-to-faces tasks allowed us to explore at least two of these phases: the competitive interaction among the accessed semantic alternatives and the selection of one of the competitors.

In line with the competitive model of selection we expected, within the ignore-faces condition, that the two professions associated with the faces were automatically encoded and interacted with each other in a mutual suppressive way (Kan IP and SL Thompson-Schill, 2004, 2004; Wheatley T et al., 2005). As selection in this case was not involved, we predicted that the task demand for related and unrelated condition was the same. Therefore, the fMRI signal in IFG would be modulated only by differences in the amount of competition between the ignore-related-faces and ignore-unrelated-faces. We expected that this mechanism was controlled by the interaction at the neural level of the two semantic representations. In particular, we assumed that the two faces and their associated profession would activate a set of distributed neurons that were interconnected to each other. In analogy with models of visual representation (Jiang X et al., 2006; Loffler G et al., 2005; Op de Beeck HP et al., 2008; Tanaka K, 2003), two semantically related representations might have stronger overlap in activity patterns compared to two unrelated entries (Oppenheim GM et al.). This in turn would result in stronger inhibitory connections for professions belonging to the same category compared to different categories, leading to a lower fMRI signal for related vs. unrelated condition (within-category inhibition). We would expect the opposite pattern if inhibition was stronger between two category-nodes (between-categories inhibition).

We expected that the IFG would also participate in the selection of the relevant profession in the attend-to-face condition. Kan IP and SL Thompson-Schill (2004) showed that the IFG was actively involved in a large number of tasks where the selection demand was systematically varied. However, as already pointed out, it is still not clear whether the modulation of the IFG is related to the amount of competitive alternatives associated with the stimuli presented (Kan IP and SL Thompson-Schill, 2004, 2004; Milham MP et al., 2001; Thompson-Schill SL et al., 1997) or rather to the resolution of the competition among these alternatives. In the former case we would expect a larger fMRI signal when the target face had to be selected in a more competitive environment. Alternatively, if IFG is recruited when selection is easily accomplished, as suggested by Grindrod et al. (2008), we would expect an enhancement of activation in IFG when competitive interactions between the two conceptual representations (target-distractor) are reduced. A third possibility is related to a bias mecha- 
nism similar to the one proposed for the visual domain. In this case, we would expect that attention to one face resolved the competition and biased the neural processing towards the relevant semantic information. This would result in a lack of relatedness effect in the attendto-face condition in the IFG reflecting a successful semantic selection.

The attend-to-face condition was also used to explore whether FFA would be engaged in semantic selection. This region has been shown to be relevant for face processing and to be involved in accessing semantic information related to faces (Bruce $V$ and A Young, 1986; Galdo Alvarez $S$ et al., 2009). As described above, we already investigated the role of FFA when a target face had to be selected in the context of another face according to its visual features. The present experiment gave us the opportunity to further investigate the role of FFA when selection involved both semantic and face processing. In particular, we tested whether the semantic information was integrated in FFA and whether this region was involved in semantic access and selection.

Recent $\mathrm{fMRI}$ studies observed that the fusiform gyrus was modulated by the semantic relatedness between pictures that were repeatedly (Wheatley T et al., 2005) or simultaneously presented (Hocking J et al., 2010). Hocking et al. (2010), for example, presented in a post-cue paradigm two line drawings that where spatially overlaid and characterized by two different colors (red and green). The objects belonged to the same or different semantic category (e.g. cat and dog, or cat and table). The display was followed by a color cue. The subjects were instructed to name the object related to that cue. The authors showed that the fusiform gyrus was more activated for semantically related compared to unrelated visual stimuli. They suggested that the correct association between the cue and the corresponding object was necessary to accurately perform the task. Moreover, in line with the prelexical account of semantic interference, the integration between perceptual and semantic information, occurring in the fusiform gyrus, is more demanding when the two objects belonged to the same category. This in turn required additional attentional resources which might increase the $\mathrm{fMRI}$ signal for related vs. unrelated objects in the fusiform gyrus (Dean MP et al., 2001).

In line with these findings we also expected in the present experiment a modulation of the fusiform gyrus by relatedness when one of the faces had to be selected according to a specific profession (attend-to-face condition). In particular, we expected that in an early stage of semantic selection, prior to its resolution in the IFG, FFA would access the profession associated with the target face and integrate this information with its perceptual features (Galdo Alvarez S et al., 2009). In addition, we expected that the presence of the distracting face would interfere with this integrative mechanism in a larger extent when the two faces were semantically related. The attentional system would, in this case, enhance the FFA activity in the related condition in order to solve the semantic interference and make the selection as accurate as possible in a more demanding situation (Dean MP et al., 2001; Hocking J et al., 2010). 


\section{Methods}

\subsection{Subjects}

Fourteen healthy volunteers ( 9 female, mean age $=24.4$ years, $S D=3.4$ years) participated in the experiment. They were all native Dutch speakers and they had normal or corrected-tonormal vision. After the explanation of the procedures, participants signed an informed consent. Subjects were paid or received 'credit points' as compensation for their participation to the experiment. The study was approved by the ethical committee of the faculty. For two subjects head-movement inside the scanner was significantly supra-threshold. We therefore excluded them from the statistical analysis. In addition, the FFA localizer was performed for 8 subjects (out of 14 scanned).

\subsection{Stimulus preparation}

The stimuli consisted of two sets of 8 pairs of faces. One set represented faces that were semantically related (REL). The other set represented faces that were semantically unrelated (UNREL) (Figure 3.a). The faces in the pairs were selected from a large set of pictures of faces (total of 60 pictures) provided by Mark Steyvers (http://psiexp.ss.uci.edu/research/software.htm). The pictures were all taken under similar lightning condition with neutral facial expressions (Kayser S, 1985).

The semantic relatedness between faces in a pair was defined by assigning each face to a profession that belonged to the same or to a distinct profession-category. In total there were 4 categories and 4 professions per category. These 16 different professions were associated with the two sets of face pairs (REL, UNREL). The profession categories consisted of 'sporters' (sportsmen), 'horeca' (hotel and catering industry), 'doktoren' (physicians) and 'arbeiders' (workmen). In a pretest, another set of subjects ( $N=15,7$ females, mean age = 35.7) were asked to assign as many professions as possible to the 4 categories in a limited amount of time (60 seconds). The final set consisted of those professions that most of the subjects identified as representative for a particular category. In particular 'tennisser' (tennis-player) 'voetballer' (football-player) 'wielrenner' (biker) 'zwemmer' (swimmer) belonged to the 'sporters' category, 'huisarts' (house-doctor) 'tandarts' (dentist) 'kinderarts' (pediatrician) 'oogarts' (ophthalmologist) constituted the 'doktoren' category, 'ober' (waiter) 'chefkok' (cook) 'café'-baas' (bar-owner) 'bar-man' belonged to the 'horeca' category and 'metselaar' (mason) 'tuinman' (gardener) 'electricien' (electrician) 'loodgieter' (plumber) were chosen as 'arbeiders' professions.

In order to control for any visual similarity (or dissimilarity) between faces in a pair, they were both dissimilar at the level of visual features. Dissimilarity was defined by using the two major approaches adopted for face representation: the purely psychological or top-down approach and the purely computational or bottom-up approach (see Figure 1.a, b in Chapter 
2). Both approaches take into account the multidimensional representation of faces (Steyvers M, \& Busey, T. , 2000) and are described in more details in the section about "Stimulus preparation" in Chapter 2. Two faces were considered as visually dissimilar if they were labeled as such both in the top-down (subjective) as well as in the bottom-up (objective) approach (see Figure 1.c in Chapter 2). This procedure gave rise to 16 pairs of faces that were divided into two sets of 8 pairs each. In a last step, in order to avoid differences in the level of activation within FFA across conditions, we computed the distance of both sets of faces from the face considered as the mean face (Loffler G et al., 2005). The two sets were, on average, at the same distance from the mean face.

\subsection{Procedure in face-profession association phase}

Prior to the main fMRI measurement, participants needed to associate to each face a specific profession in order to perform the main experiment. This procedure consisted of two phases: a learning phase and a testing phase (Figure 1).

During the learning phase subjects were asked to associate 32 faces with 16 professions. They were presented with a single face in the centre of the screen and their associated profession was typed below the picture. When the subject learned the face-profession association, the next face was presented via self paste button press. After finishing the first training run, participants were asked whether they wanted a re-test or to proceed with the next phase where we tested the level of their association.

In the testing phase participants saw a face in the centre of the screen for 5 seconds, and they had to select the corresponding profession among 4 options displayed below the target ( 1 correct and 3 randomly chosen among the remaining 15). In order to reinforce the learning of the face-profession association, the subject's responses were followed by a correct association feedback. Blocks of 32 faces were presented. The amount of training blocks depended on the subject's performance. Participants started the main experiment in the fMRI scanner when they reached $85 \%$ correct responses during the testing phase. 
learning phase

GOAL: 32 faces

to associate with 16 professions

\section{testing phase}

GOAL: $85 \%$ correct responses

in two consecutive blocks

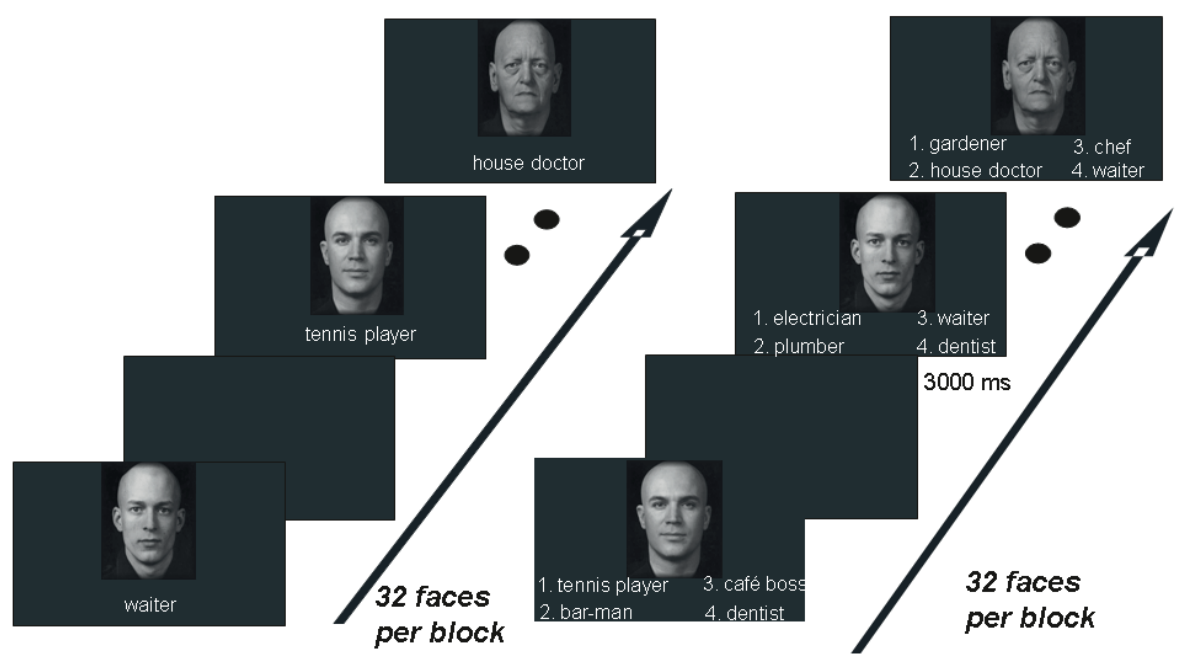

Figure 1. Learning and testing phase of face-profession associations.

\subsection{Procedure in the main experiment}

The main experiment consisted of two runs in a block-design (Figure 2). Each run consisted of 32 blocks and started with a blank screen with a white cross in the centre lasting for 8 seconds. A block consisted of 18 trials. A single trial started with a $500 \mathrm{~ms}$ of blank screen, followed by a pair of faces presented for $500 \mathrm{~ms}$. The two faces were positioned in opposite hemifields and they were either REL or UNREL to each other at the semantic level (see Stimulus preparation). The single pictures subtended approximately $6.5^{\circ}$ (height) $\times 5.4^{\circ}$ (width) of visual angle and were aligned with the centre of the screen. The entire visual display covered $6.5^{\circ}$ (height) $\times 12.2^{\circ}$ (width) of visual angle.

The display size was designed to be comparable with those used in previous studies on face processing in order to guarantee that FFA integrated the two simultaneously presented faces within each experimental trial (Gauthier I et al., 2000; Halgren E et al., 1999; Kanwisher N et al., 1997; Rossion B et al., 2003; Steeves JK et al., 2006; Wojciulik E et al., 1998). Between the faces, two bars $\left(0.2^{\circ}\right.$ in height) were presented $0.15^{\circ}$ above and below the centre of the visual screen. A block of trials ended with a resting condition consisting of a blank screen lasting for 10 seconds. A visual instruction was presented at the beginning of each 
block for 3 seconds and it was followed by a white cross on a blank screen for 10 seconds after which the trial started. The instruction signalled the type of task to be performed in the next block.

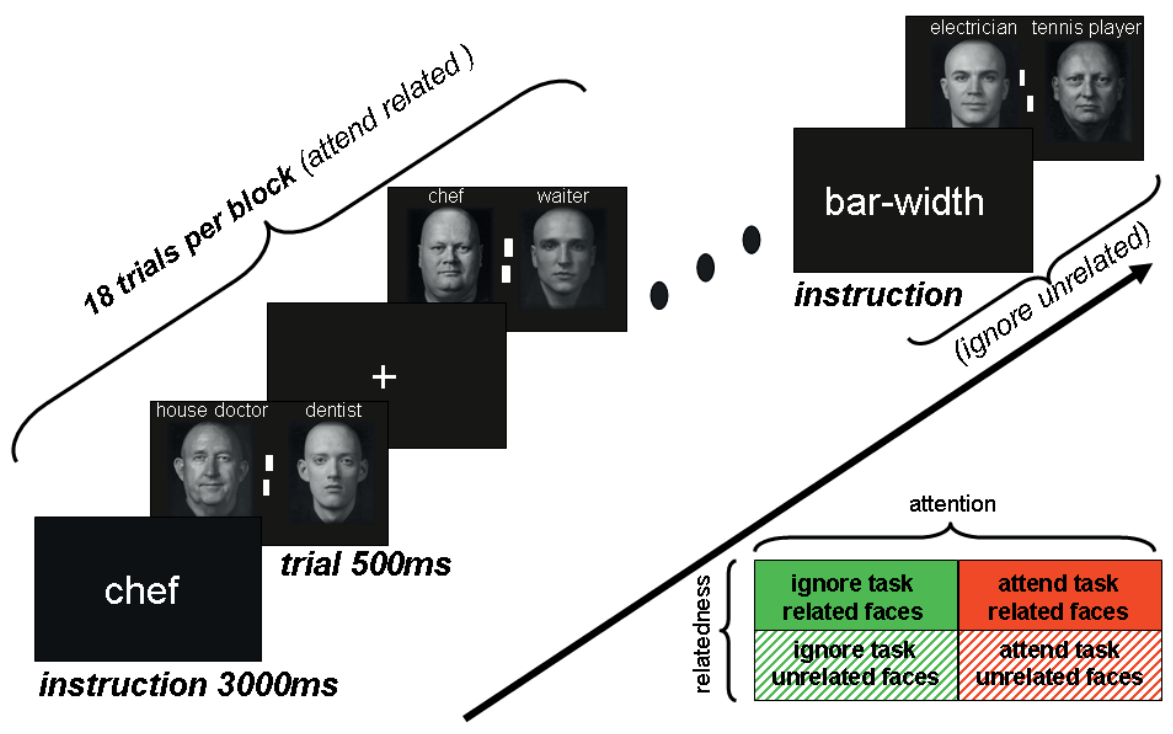

Figure 2. Experimental design. Block structure with examples of two different conditions (attend-to-faces with related display and ignore-faces with unrelated display).

For the ignore-faces (IGN) task, two keywords ('streep-breedte', 'bar-width') centred on the screen, instructed the subject to perform a bar-width discrimination task in the centre of the display, while ignoring the background (for a detailed description of the task see Figure 3.a and "Procedure in the main experiment" in Chapter 2). For the attend-to-face (ATT) task, subjects had to perform a match to sample task. The instruction consisted of a letter string indicating a profession, again displayed in the centre. For each subsequent trial in the block, participants were asked to respond via a button press when the profession associated to the face presented on the left side of the screen matched the one presented in the instruction phase (Figure 3.a). For this task, subjects were instructed beforehand to avoid saccades and to covertly attend to the item on the left while fixating the centre. For each task and within each block, targets (matches) occurred on average $20 \%$ of the time. In analogy to preceding studies (Bles M and BM Jansma, 2008; Bles M et al., 2006; Gentile F and BM Jansma, 2010) targets always occurred at the target position. In both tasks, the time for the subject to respond was $1000 \mathrm{~ms}$ (trial duration). 
The design was a $2 \times 2$ repeated measures design with factors attention (ATT, IGN) and relatedness (REL, UNREL) leading to 4 conditions (IGN_REL, IGN_UNREL, ATT_REL, ATT_UNREL, Figure 3.b). The participants were not made aware of the relatedness manipulation. The order of the blocks was randomised and counterbalanced across participants and runs by means of the Williams design (Williams EJ, 1949). This scheme is a generalized Latin square design, balanced for first-order carryover effects. The stimulus display was identical for both types of task. Participants were instructed to fixate the centre of the screen throughout the experiment and to minimise head and eye-movements while in the scanner.

a)
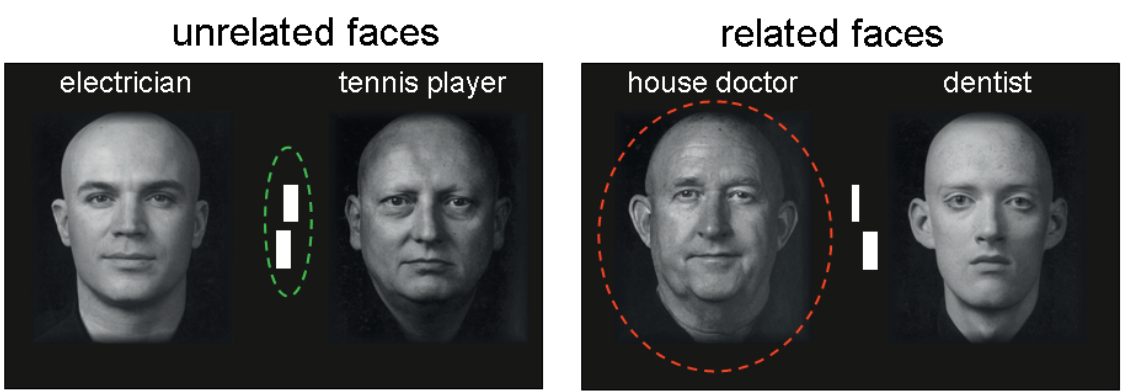

ignore task

attend task

b)

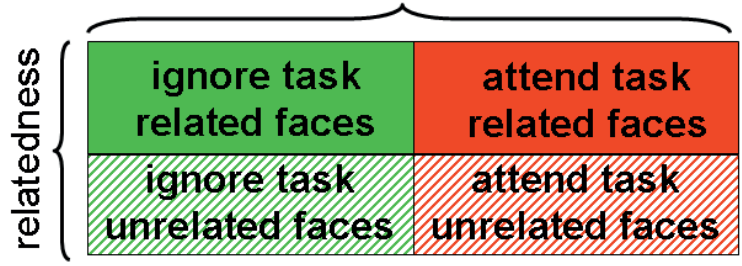

Figure 3. Tasks and stimuli. (a) Examples of the two types of the display presented (unrelated and related faces) for the two tasks (attend-to-face and ignore-faces). Note that in the main experiment profession labels (words) were not presented. (b) Conditions: $2 \times 2$ repeated measures design. 


\subsection{Data acquisition}

Images were acquired on a 3T Siemens Magnetom Allegra head scanner (Siemens Medical System, Erlangen, Germany) using a standard head coil. Thirty-two oblique axial slices (inplane resolution: $3.5 \mathrm{~mm} \times 3.5 \mathrm{~mm}$, slice thickness: $3.5 \mathrm{~mm}$, interslice distance $0 \mathrm{~mm}$ ) covering the entire cortical volume were acquired using an echo planar imaging sequence (repetition time $(T R)=2000 \mathrm{~ms}$, echo time $(T E)=29 \mathrm{~ms}$, matrix: $64 \times 64)$. We acquired 675 volumes per run and discarded the first two from the analysis due to the T1 saturation effect. Functional slices were aligned to a high resolution 3D anatomical dataset acquired in the middle of the entire session and consisting of 176 slices (the ADNI sequence was used: $T R=2250$ $\mathrm{ms} ; \mathrm{TE}=2.6 \mathrm{~ms}$; flip angle $=9^{\circ}$, voxel dimension $=1 \times 1 \times 1 \mathrm{~mm}^{3}$ ).

The participants were placed comfortably in the scanner and their heads were fixated with foam pads. Participants saw the stimuli projected on a screen through a mirror mounted on the head coil. The visual field was perceived at a distance of 57 centimeters. The stimuli were delivered using the Presentation 12.2 package (Neurobehavioral Systems, Inc. www.neurobs.com). Stimulus presentation was synchronized with the MR data acquisition by triggering the stimulus program with the first MR pulse.

\subsection{Analysis}

Both the functional and the anatomical data were analyzed using the BrainVoyager QX package (Brain Innovation B.V., Maastricht, The Netherlands). The anatomical scans were used to project the statistical results from the functional data onto high-resolution anatomical images. They were also used to derive the Talairach coordinates needed to normalize both sets of data.

Functional data were pre-processed, aligned to the anatomical images, and normalized. The pre-processing procedure started with correcting the data for motion artefacts in three dimensions and for slice scan-time differences. The fMRI data were also spatially smoothed with a $4 \mathrm{~mm}$ FWHM Gaussian kernel, and linear drifts were removed from the signal. Finally, data were high-pass filtered to remove slow frequency drifts up to 9 cycles per time course. After the pre-processing, functional data were aligned to the high-resolution anatomical images and normalized to the standard 3-dimensional Talairach space. The final version of the functional data consisted of a 4-dimensional $(x, y, z, t)$ dataset in Talairach space for each run and participant.

After digital brain normalization, the statistical analysis from multiple subjects was performed via the General Linear Model (GLM) with a fixed effects (FFX) implementation. In this approach subjects and runs were collapsed together. The beta values from this unique dataset were computed for each condition and entered a 2×2 ANOVA with factor attention and relatedness. In addition, a random effects (RFX) analysis was performed with subject considered as the random variable (see "Analysis" in Chapter 2, for more details). In both ap- 
proaches, the effects were investigated in conjunction with contrasting all the conditions versus baseline.

Both FFX and RFX-GLM were carried out in the whole brain. We also performed a region of interest analysis (ROI) within FFA via FFX-GLM. We did not perform a RFX-GLM analysis in this case due to the limited amount of subjects (8) in which we localized FFA. For the wholebrain analysis, correction for multiple comparisons was performed using cluster-size thresholding (described in more details in the "Analysis" paragraph in Chapter 2). The correction for multiple comparisons was not performed for the ROI analysis in FFA. In fact, in this case, the beta values of the fMRI signal change per subject and condition were extracted, averaged over all voxels in the single-subject volume-of-interest (VOI), and evaluated (summary statistics, level 1).

\subsection{Localization of FFA}

An independent functional run, consisting of 324 volumes, was used to localize FFA in 8 subjects (see "Localization of FFA", in Chapter 2, for details).

\section{Results}

In this section, we will present the behavioral and fMRI results. For sake of clarity, the findings discussed in the present paper will mainly regard the results from the RFX analysis. The FFX analysis was used to perform a region of interest (ROI) analysis in FFA (see ROI analysis in FFA). It also helped to identify, in the whole-brain implementation, spots in the IFG sensitive to the relatedness manipulation in order to further investigate this areas with a ROI-RFX analysis (see Whole-brain GLM: FFX analysis).

\subsection{Behavioral Results}

We computed error rates (ERs) and reaction times (RTs) on the ignore-faces (IGN) and attend-to-face (ATT) task for the related (REL) and the unrelated (UNREL) condition. Mean ERs and RTs were then submitted to a repeated measures ANOVA with two factors, namely attention and relatedness. For RTs, a significant main effect of attention was observed ( $F(1$, 13) $=45.635, \mathrm{p}=.000$, mean reaction time ATT $=639 \mathrm{~ms}, \mathrm{SE}=14$, mean IGN $=563 \mathrm{~ms}, \mathrm{SE}=$ 12) but no effect of relatedness $(F(1,13)=.317, p=.583$, mean $R E L=604 \mathrm{~ms}$, SE $=12$, mean UNREL $=599 \mathrm{~ms}, \mathrm{SE}=13)$ or interaction $(F(1,13)=.076, p=.787)$. For ERs we found a significant main effect of relatedness $(F(1,13)=6.858, p=.021$, mean $R E L=16 \%$, SE $=1$, mean UNREL $=14 \%, S E=1)$ and a significant interaction effect $(F(1,13)=4.782, p=.048)$ but no main effect of attention $(F(1,13)=.280, p=.606$, mean ATT $=15 \%$, SE $=1$, mean IGN $=16$ $\%, S E=2$ ). This interaction effect reflected a relatedness effect only in the attend-to-face ( $p$ $=.002)$ and not in the ignore-faces condition $(p=.882)$. 


\subsection{ROI analysis in FFA}

A FFX-GLM analysis restricted to FFA (Figure 4) revealed, bilaterally, a main effect of attention (ATT vs. IGN) (right FFA: $p=.000$, left FFA: $p=.000$ ). In addition we observed within the right hemisphere a significant main effect of relatedness (REL vs. UNREL) ( $p=.000)$.

We further investigated the nature of this relatedness effect by testing it independently for the attend-to-face and ignore-faces condition in two separate pairwise comparisons. We observed a larger fMRI signal when related faces were presented compared to unrelated faces both in the attend-to-face and ignore-faces condition (ATT_REL > ATT_UNREL: $p=.002$, IGN_REL > IGN_UNREL: $p=.005)$.

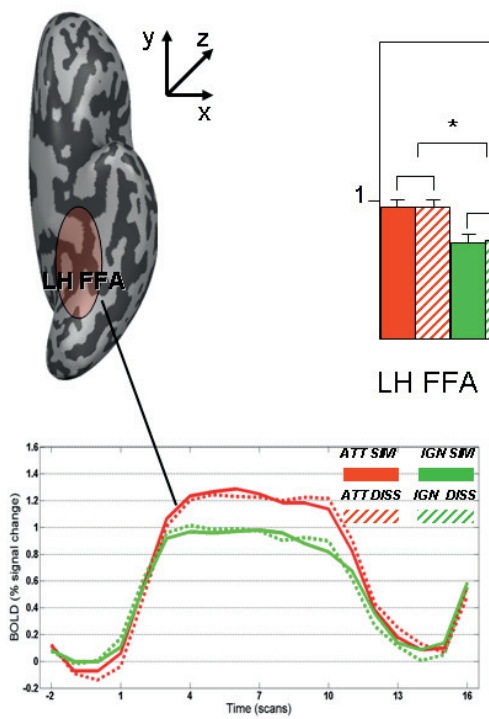

beta values

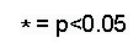

$*=0<0.05$
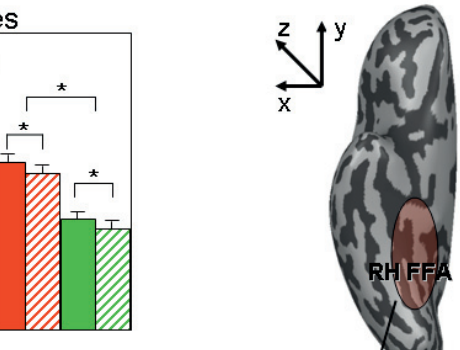

H FFA

RH FFA

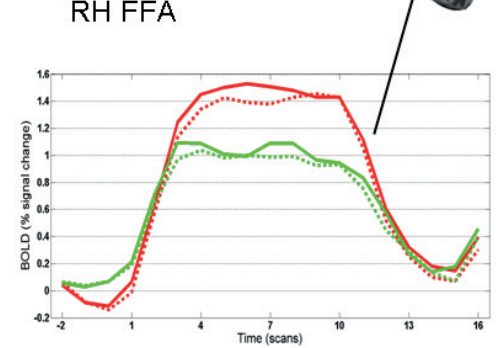

Figure 4. Results of the RFX-GLM analysis in FFA (region of interest analysis) separately for left and right hemisphere. In the central column the beta weights of the GLM analysis are displayed. The mean time courses of the fMR signal change per condition and per region of interest are also shown. 


\subsection{Whole-brain GLM: FFX analysis}

The FFX analysis revealed a large network of regions significantly more activated for the attend-to-face compared to the ignore-faces tasks (ATT vs. IGN). In particular, we distinguished frontally a collection of areas comprising the anterior part of the insula, the inferior frontal lobe and a fraction of the central part of the middle frontal gyrus (MFG) ( $p=.009$, corrected). The task effect was also observed within the precentral gyrus and sulcus and in correspondence to the caudal part of the superior frontal gyrus (SFG) ( $p=.009$, corrected). In the parietal lobe, the bilateral intraparietal sulcus and the entire superior parietal lobe also showed higher fMRI signal for ATT vs. IGN tasks ( $p=.009$, corrected). The difference between these tasks was also significant bilaterally within the middle occipital gyrus and along the entire fusiform gyrus and collateral sulcus ( $p=.009$, corrected). In addition, limited to the right hemisphere, the postcentral gyrus and the posterior part of the temporal lobe showed a differential activation for attend-to-face vs. ignore-faces tasks $(p=.009$, corrected).

The main effect of relatedness was significant bilaterally within the central part of the IFG and the inferior occipital gyrus (IOG) ( $p=.009$, corrected). In particular, these regions showed an enhancement of $\mathrm{FMRI}$ signal when faces were semantically related compared to unrelated faces regardless of the nature of the task. The same effect was observed on the right hemisphere within the intraparietal sulcus and in the posterior part of the SFG ( $p=$ .009, corrected).

The relatedness effect was also tested independently for each task via pairwise comparisons. We found a significant related vs. unrelated effect within the ignore-faces condition bilaterally in the IFG ( $p=.009$, corrected). The same modulation was observed within the right hemisphere in the SFG, MFG, middle temporal gyrus (MTG) and IOG ( $p=.009$, corrected). The same effect was not significant when we compared related with unrelated faces for the attend-to-face task.

\subsection{Whole-brain GLM analysis: RFX analysis}

The whole RFX analysis (Figure 5) confirmed a significant main effect of task in few of the areas identified by the FFX-GLM. In particular, the attend-to-face task elicited a larger fMRI signal compared to the ignore-faces task bilaterally within the anterior insula, the precentral sulcus, the caudal SFG and along the fusiform gyrus ( $p=.009$, corrected). Restricted on the right hemisphere we found the same effect in the inferior frontal sulcus, in the precentral gyrus and sulcus ( $p=.009$, corrected). In addition, we observed a main effect of relatedness (related > unrelated) within a spot localized in the IFG ( $p=.009$, corrected) but no interaction of factor attention and relatedness. 


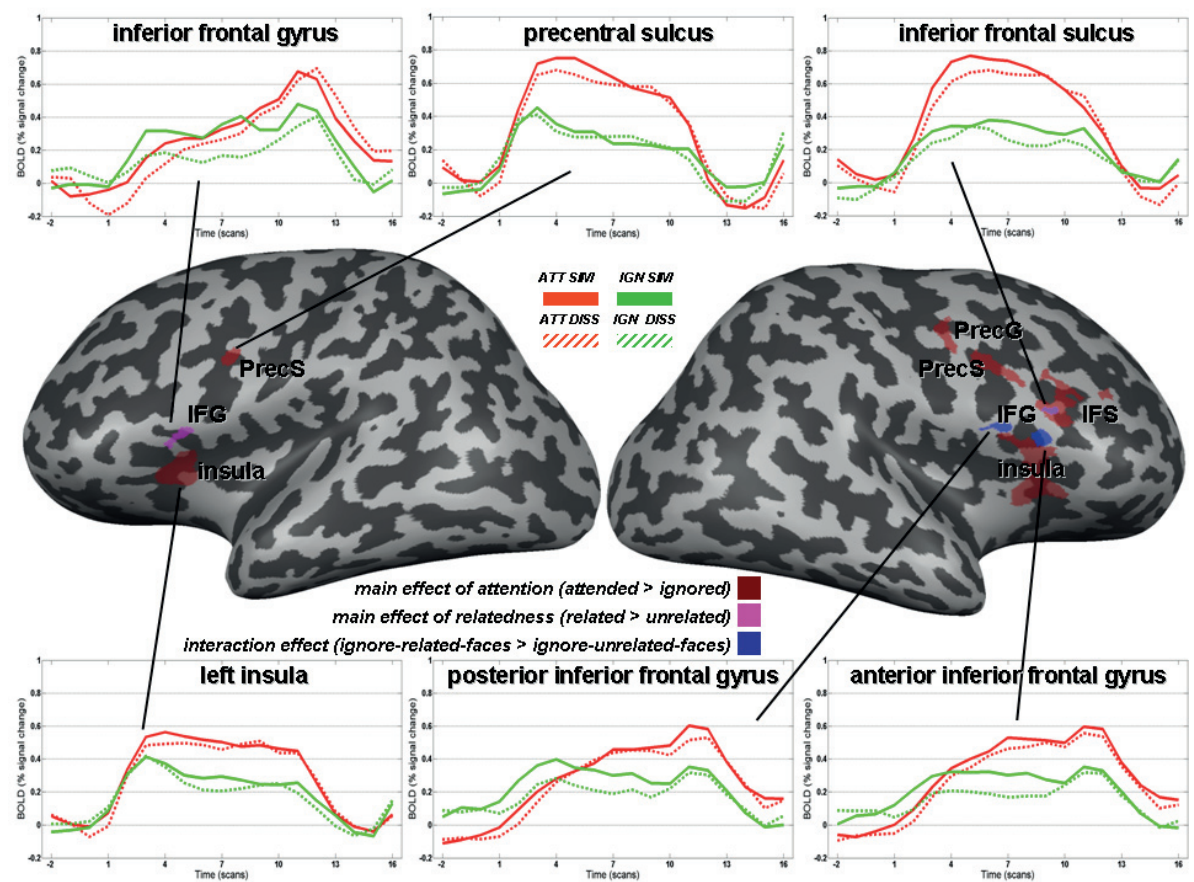

Figure 5. Results of RFX-GLM analysis in frontal areas (whole brain analysis), separately for left and right hemisphere. The mean time courses of the fMRI signal change per condition and per region of interest are displayed.

Due to its relevance in semantic processing, we further investigated the IFG via ROI-based analysis (Bookheimer S, 2002). However, the lack of a proper IFG functional localizer did not allow us to define a region selectively responsible for semantic selection. On the other hand, the FFX analysis revealed that IFG was significantly sensitive to the semantic manipulation. Therefore, the region of interest was selected by considering those spots within the left and right IFG that showed a relatedness effect in one or both tasks within the FFX analysis.

For the right IFG, this analysis revealed a main effect of attention $(p=.000)$ and relatedness $(p=.012)$ and an interaction effect $(p=.011)$. A pairwise comparison within the ignorefaces condition showed a significant difference between related and unrelated faces (IGN_REL > IGN_UNREL: $p=.004)$. The same comparison did not reach significance within the attend-to-face condition. Within the left IFG, we only found a main effect of relatedness ( $p=.03)$. 


\section{Discussion}

In the present study we investigated the mechanism of semantic selection in the context of face processing. In the visual domain, the process of selecting a relevant item in a cluster of several objects has been modeled as an attentional bias mechanism. Such a bias originates from a fronto-parietal network and "solves" the competitive interactions occurring among all the alternatives in favor of the encoding of the target. In line with this model we recently showed that two faces that were simultaneously presented and ignored actually compete for representation. We also observed that attention to one face biased neural competition towards the processing of that face only (Gentile F and BM Jansma, 2010).

Kan and Thompson-Shill (2004) proposed that the semantic selection of a visual stimulus can also work according to a similar competitive model of selection. In line with psycholinguistic theories of lexical access, they suggested that when an item is presented, several semantic representations related to the stimulus become activated and, in turn, interact with each other in a competitive manner (Levelt WJM et al., 1991). They also showed that selection of one of those representations is affected by the amount of neural competition. In this context, Kan and Thompson-Shill (2004) investigated the role of the IFG in semantic selection. However, this was done by using experimental designs where competitive interactions and selection mechanism were merged together.

The present experiment aimed to disentangle the two mechanisms by exploring these processes separately. In particular we tested whether the semantic information associated with two simultaneously presented faces interacted in a competitive manner and to which extent attending to one of the faces facilitate the selection of the relevant information. In analogy to the experimental design in Chapter 2 and Chapter 3 we investigated the competitive interactions between different semantic representations by using an ignore-faces task where the subjects were instructed to ignore the faces in the background. We varied the amount of semantic representations by presenting pairs of faces that were associated with professions belonged to the same category (related faces) or to two different categories (unrelated faces). The selection mechanism was tested with an attend-to-face task where participants had to perform a match-to-sample task on one of the two faces.

\subsection{The IFG}

In the IFG, we found an interaction of the factors attention and relatedness reflecting a significant relatedness effect within the ignore-faces and not in the attend-to-face condition. The modulation in the ignore-faces task indicated that professions associated with the two faces were encoded though both ignored. Therefore, in line with the idea of Kan and Thompson-Shill (2004), the visual stimulation of the two faces elicited an automatic coacti- 
vation of the corresponding semantic representations in the IFG. A pairwise test revealed a lower IFG activation in the ignore-faces task when unrelated compared to related faces were presented. If we assume that a smaller fMRI signal represents stronger competitive interactions between the two professions, the relatedness effect in the ignore-faces condition suggested that unrelated faces competed for representation more than related faces. This might reflect a category competition effect. In fact, the activation of a category-node could give rise to the automatic coactivation of the all set of the connected nodes representing the professions belonging to that category. This would lead to a higher amount of activated semantic representations (professions) in the unrelated (two categories) compared to the related condition (one category) resulting in a stronger competition. Alternatively, the observed IFG pattern in the ignore-faces condition might represent the ongoing state of coactivation of the two semantic representations. According to theories of lexical access (Oppenheim GM et al., 2010) this mechanism would be more demanding in case of the processing of professions from the same category hence leading to a larger activation in the IFG.

Interestingly, when one of the faces was to be attended to, the IFG did not reveal the relatedness effect we observed when both faces were to be ignored. A similar effect was observed when the ambiguity related to multiple-representations stimuli was reduced (or biased) towards a specific semantic representation (Kan IP and SL Thompson-Schill, 2004; Kremin $\mathrm{H}$ et al., 2000; Levelt WJM et al., 1991). Our results suggested that attention was able to mimic this bias effect by filtering out the related as well as the unrelated distracting face and restricting the semantic processing towards the information of the target face alone. The same lack of similarity modulation was found in FFA when a face was selected according to its physical features while presented together with a similar or dissimilar (in visual terms) face (Gentile F and BM Jansma, 2010). Therefore, the underlying mechanism responsible for face selection might be the same both when the face is selected on the basis of its visual characteristics (in the FFA) or according to specific semantic information (in the IFG).

In conclusion, the pattern of activation in the IFG suggested that in semantic processing the mechanisms of neural competition and selection are functionally distinct. In fact, we observed that the semantic representations of the two faces actually interacted with each other only when they were both ignored. When only one of the faces was attended to, the influence of the distracting face was cancelled out and selection was, most likely, accomplished at that moment.

\subsection{The FFA}

Another goal of the present experiment was to identify additional cortical regions involved in semantic selection of faces associated with specific professions. We restricted our area of investigation to the FFA due to its central role in face processing and its possible involvement in the initial stage of the integration of semantic information. We localized FFA with an independent functional localizer and we performed a region of interest ANOVA with factors 
attention and relatedness. We observed a main effect of relatedness within the right FFA. This effect revealed a significant difference between the related and the unrelated condition both in the attend-to-face and ignore-faces tasks. In particular, within the attend-to-face condition FFA showed a larger fMRI signal for the related compared to the unrelated condition. In a recent fMRI study, Hocking et al. (2010) found a similar pattern when they investigated the influence of distractors on the semantic selection of a relevant item. They presented two superimposed semantically related or unrelated objects and asked the participants to select one of them based on a specific cue. They observed within the fusiform gyrus a larger fMRI signal when the object to be selected was semantically related to the distractor. According to Dean MP et al. (2001) the integration between the cue and the target stimulus is crucial for selection. They also suggested that this integration process can be hampered by distractors that are related to the target. The directionality of the relatedness effect in the Hocking et al. (2010) study supported this idea as they showed a stronger semantic interference for related compared to unrelated distractors. In particular, they interpreted the larger fMRI signal for the related vs. the unrelated condition as an attentional effect needed to amplify the representation of the target object in a more interfering context (semantically related distractor)

In line with the semantic interference effect, we found at the behavioral level, that the attend-to-face task was more demanding when the profession associated with the attended face was semantically related to the distractor than when it was unrelated. This result in combination with the relatedness modulation in the attend-to face condition in FFA suggested that the irrelevant face was actually encoded at the semantic level and interfered with the processing of the relevant information related to the target face. Moreover, FFA is considered the region where the integration of semantic and physical face features occurs. In fact, according to the model of Bruce and Young (1986) in FFA the recognition of a face takes place via face recognition units (FRUs). The access to any semantic information related to a specific face is accomplished by the connection of the FRUs to person identity nodes (PINs). If two faces are semantically related, two different FRUs might access the same PIN, making the actual recognition of the target face more demanding compared to the unrelated condition. Therefore, in line with Hocking et al. (2010)'s interpretation, an additional boost of attention is required in the related condition to facilitate the integration of the physical and semantic features of the target face. This, in turn, would most likely permit the correct selection of the target in a later stage of semantic processing in the IFG where semantic selection is achieved (reflected by the lack of relatedness effect in the attend-to-face condition).

Similarly to the IFG, FFA showed a relatedness effect within the ignore-faces condition. In particular, we observed a lower fMRI signal for unrelated-ignored-faces vs. related-ignorefaces condition. In line with the interpretation of the pattern of the IFG in the ignore-faces task, this result would indicate that semantic information associated with the two faces competes in FFA for representation. However, FFA is not responsible for the semantic proc- 
essing per se. Therefore, we rather suggested that the relatedness effect in FFA resulted from a top-down modulation from the IFG.

In summary, the present experiment showed that the selection of relevant semantic information associated with a face occurs with the same modality of selecting a face according to its visual features. In fact, the results in the IFG suggested that when the two faces were irrelevant to the task performed (ignore-faces condition), their associated professions were actually both encoded and competed for representation. Attention to the profession associated with the relevant face biased the competition towards the processing of that profession only.

In addition, when one of the two faces was attended to, we observed in FFA a larger fMRI signal for related compared to unrelated faces. In line with the semantic interference effect reported in psycholinguistic literature, these results suggested that the integration of semantic and perceptual information was more demanding in the related case. This in turn led to a stronger engagement of the attentional system which enhanced the activation in FFA in order to correctly perform the task in the most difficult condition (attend-to-relatedface). The interference account in FFA was supported by the behavioral results that confirmed that the attend-to-related-face was more demanding compared to the attend-tounrelated-face. The interference effect, we observed only in FFA and not in the IFG, also suggested that the inhibition effect from related distractors, that are often reported in behavioral studies on semantic selection (Damian MF et al., 2001; Oppenheim GM et al., ; Vigliocco G et al., 2002), might actually reflect a mechanism that is not strictly related to selection but rather to an earlier stage of processing, i.e. the access of multiple candidate prior to selection.

In conclusion, our fMRI study showed that the IFG and FFA played a crucial role in the semantic selection of a face and that within this process both areas performed functionally distinct subroutines. Although an accurate temporal description of the neural events occurring in the present experiment is far beyond the temporal resolution of $\mathrm{fMRI}$, our results suggested that at an initial stage of semantic access eventual interferences from distractors are solved in FFA. In a later stage this information is sent out to the IFG where the integration of the face and a specific profession is accomplished and where attention is able to bias the competition between two semantic representations towards the relevant information only (selection). In Chapter 5 we further investigated this dynamic framework by using electroencephalography in combination with the independent component analysis. 


\section{Acknowledgments}

The authors wish to thank Job van den Hurk for helpful discussions. This project was supported by Maastricht University (HRM initiative Mobilizing Minds: "Females into higher positions") to B.M. Jansma. 

Semantic selection in the context of face processing: an EEG study 


\section{Abstract}

When we engage in a conversation with someone, an efficient and accurate retrieval of the information related to this person plays a central role in the dynamics of the discourse. In a recent $\mathrm{fMRI}$ study (Chapter 4 ) we suggested that an important aspect to accomplish this goal is a correct association of the facial and semantic features of the speaker. We also pointed out that an appropriate selection of the relevant target and filtering out irrelevant information from the context (e.g. related to surrounding people) is necessary. The context, in that case, was represented by another face associated with a profession that could belong to the same or different semantic category of the target face. The subjects' task was either to match one of two faces with a specific profession sample (attend-to-face condition) or to ignore both faces by performing a distractor task (ignore-faces condition). We observed that both the inferior frontal gyrus (IFG) and the fusiform face area (FFA) contributed to the semantic selection of a face in context but in different ways. In particular, the modulation by semantic relatedness within the IFG in the ignore-faces condition was interpreted as a coactivation of the semantic representations (professions) of both faces. The direction of this effect (ignore-related-face > ignore-unrelated-faces) suggested the occurrence of a semantic competition mechanism between the two faces. When one of the two faces was attended to, the competitive interactions were biased towards the processing of the relevant semantic representation (no relatedness effect in attend-to-face condition). The pattern of activation in FFA (attend-to-related-face $>$ attend-to-unrelated-face) suggested that the distracting face inhibited the processing of the target and that attention compensated for this effect by enhancing the activation in the most interfering context (attend-to-related-face). In the present study we investigated the temporal dynamics of the neural events in the FFA and IFG by using electroencephalography (EEG) in combination with the Independent Component of Analysis (ICA). We used the same experimental design as in the fMRI study. ICA identified four clusters of independent components (ICs) that we defined as the N170, N250, N300 and N400. In line with the semantic interference effect we found in FFA with FMRI, the N250 was modulated accordingly in the attend-to-face condition. This result supported the idea that the semantic interference mechanism occurred at an early stage of visual-semantic processing of faces (around $250 \mathrm{~ms}$ ) in correspondence of the integration of these two types of information in FFA. The N300 showed a relatedness effect in the ignore-faces condition similar to that observed within the IFG (fMRI study). This indicated that semantic competitive interaction between the two professions takes place in the IFG around $300 \mathrm{~ms}$. An opposite pattern of relatedness modulation was found in the same time-window also in the attend-to-face condition suggesting a residual inhibition from the context. At the level of the N400 component, the lack of any context effect suggested that, at this stage of semantic processing, attention to the target face solved both interference and competition leading to the final selection of the relevant information. 
Keywords: semantic selection, face perception, EEG, ICA, semantic relatedness, N170, N250, N300, N400 


\section{Introduction}

In everyday life, we encounter a large amount of people. The way we deal with each of them is guided by "who they are". Therefore, a successful social interaction requires an accurate and efficient identification of the biographical information of those persons. In addition, social interactions normally occur in the context of other people whose presence can "disturb" or more in general influence our communication skills. In Chapter 2 and Chapter 3 of this thesis, we showed that the visual system encodes the perceptual features of these "background-faces" that were simultaneously presented though ignored. In addition, we showed that those faces compete for representation in the fusiform face area (FFA) in a 250 ms time-window, and in a larger amount when the two faces were similar to each other. In line with the biased competition account for visual selection, we also observed that attention biased the neural processing towards the target face only. And this occurred regardless of the similarity level (similar or dissimilar) between the distractor and the target (Gentile $F$ and BM Jansma, 2010).

Kan IP and SL Thompson-Schill (2004) recently drew an interesting parallel between the mechanism of selection in the visual and semantic domain. They reviewed several studies where visual stimuli, characterized by several semantic representations (name agreement), were presented. Participants were asked to select one of the possible name-options. At the behavioral level, it was consistently observed that subjects were significantly slower in naming objects characterized by a large set of name agreement compared to items with only few of them (Damian MF et al., 2001; Kremin H et al., 2000; Levelt WJM et al., 1991; ThompsonSchill SL et al., 1997; Vigliocco G et al., 2002). The model by Levelt WJM et al. (1991) states that when such tasks are performed, the visual system analyzes the object at the perceptual level and in a later stage all different semantic representations coactivate and interact in a competitive manner. Competition, in turn, negatively affects the semantic selection in a larger extent the larger the amount of semantic representation related to the target. At the anatomico-functional terms, Kan IP and SL Thompson-Schill (2004) showed that when a visual stimulus, associated with several name agreement, was presented and only one option was to be selected the fMRI signal in the IFG was higher than for objects associated with a unique semantic representation. However, when the selection task was embedded in a semantic interference design other regions were sensitive to semantic manipulations. De Zubicaray G et al. (2006), for example, demonstrated that the MTG was actively involved in a picture-word interference task. Hocking J et al. (2010) showed that the fusiform gyrus played a crucial role in a picture-picture interference-like design. More specifically, this area was modulated by the semantic relatedness between two simultaneously presented objects when one of them had to be selected with respect to a specific visual feature (i.e. color). In fact, when the semantic and perceptual information of an object needs to be integrated, as 
in this case, a semantically related distractor interferes with this process (Dean MP et al., 2001; Hocking J et al., 2010). The authors proposed that additional attentional resources solved this interference mechanism via the fusiform gyrus. This variability of regions involved in semantic selection suggested that the IFG is not the only area engaged in such a mechanism Therefore, it is still matter of debate at which level of semantic processing semantic competition is resolved by the appropriate selection of the relevant information. Another aspect that needs to be investigated is the functional separation of the "access of multiple candidates" and the "selection of only one of them". Previous studies were designed in such a way that these two mechanisms could not be disentangled. Chapter 4 and Chapter 5 aimed to investigate whether lexical access and selection actually share the same neural correlates or whether they could be functionally distinct in terms of spatial and temporal processing.

In a recent fMRI study (Chapter 4), we addressed the spatial characteristic of semantic access and selection. In particular, we disentangled the semantic competition process (access) and the semantic selection mechanism in the context of face processing. We also investigated whether the frontal selection occurring in the IFG might eventually interact at the functional level with other regions that are also crucial in semantic selection (e.g. fusiform gyrus). We simultaneously presented two faces in two different hemifields. Each face was associated with a specific profession. These associations were learned by the subjects before they took part in the main experiment. Two faces were defined as semantically related or unrelated to each other when their associated professions belonged to the same or different category respectively. The occurrence of competitive interactions was tested by an ignorefaces task where we asked the subjects to ignore both faces in the background and to perform an unrelated task in the center of the screen. In line with previous studies showing semantic processing for unattended items (Dell'Acqua R and J Grainger, 1999; Di Pace E et al., 1991; Glaser WR and MO Glaser, 1989; Tipper SP and J Driver, 1988; Tipper SP et al., 1988; Yee PL, 1991), we assumed that the semantic content of both faces would be automatically encoded in the inferior frontal gyrus (IFG). The relatedness modulation in the IFG supported the idea that the two faces were actually processed at the semantic level. Moreover, if we assumed that a lower $\mathrm{fMRI}$ signal reflected a higher degree of competition, the pattern of the relatedness effect suggested that the two representations competed more when faces were semantically unrelated. We interpreted this result as an effect of category competition. In fact, the simultaneous activation of two semantic category nodes occurring in the unrelated condition might cause the automatic coactivation of related nodes leading, in turn, to a spreading of competitive interactions that will be larger compared to the related condition.

In order to investigate the phase of semantic processing related to selection, we added to the design an attend-to-face task consisting of a match-to-profession task on one of the faces. Unlike the modulation within the ignore-faces condition we did not observe in the IFG a relatedness effect when one of the two faces was attended to (attend-to-face condition). This result suggested that attention to one face filtered out any context effect of the distrac- 
tor. Therefore, we concluded that the competitive interactions occurring within the ignorefaces condition were biased within the attend-to-face task towards the processing of the relevant profession only.

In FFA, we observed an enhancement of the fMRI signal when the target (attend-to-face condition) was paired with a semantically related face. This modulation of FFA by semantic relatedness suggested that the distracting face was automatically processed at the semantic level and that it interfered with the selection of the target face. This result supported the idea that FFA was also involved to a certain extent in semantic selection. In line with Dean MP et al. (2001) and Hocking J et al.(2010), we proposed that the integration of the featural and semantic information of the relevant face in FFA was more difficult when target and distracting professions belonged to the same category. Therefore, it is plausible that the attentional system increased activation in FFA in the most interfering case (related faces) in order to facilitate the semantic selection mechanism in a later stage of processing. The modulation of FFA by semantic relatedness also suggested that the inhibiting effect often reported in psycholinguistic experiments (Oppenheim GM et al., ; Vigliocco G et al., 2002) might actually reflect an interference mechanism occurring prior to selection.

In fact, we proposed in Chapter 4, that the semantic interference effect in FFA belonged to an early phase of semantic access where the association of the face and a specific profession started to take place. We also suggested that the final assemblage and integration of featural and semantic information as well as the selection of the relevant profession was accomplished in the IFG. However, the sluggishness of the BOLD response makes the fMRI signal not suitable to actually investigate the temporal profile of the neural events occurring during the attend-to-face and ignore-faces tasks.

In order to track these temporal dynamics, we employed in the present study an electroencephalography (EEG) experiment with the same design as we used in the fMRI study. In this experiment we also tested whether the two mechanisms of semantic competition and selection, which we functionally segregated in $\mathrm{fMRI}$, also occurred in two distinct timewindows. The EEG data were analyzed with Independent Component Analysis (ICA). The main assumption of this technique is that over a sufficient amount of time the signal on the scalp can be modeled as the sum of temporally independent sources originated in the cortex. ICA has been shown to be a valid alternative to the standard analysis of the EEG signal in several studies (Debener S, S Makeig et al., 2005; Debener S, M Ullsperger et al., 2005; Eichele T et al., 2008; Makeig S, M Westerfield, TP Jung et al., 1999; Onton J et al., 2005). In the EEG study described in Chapter 3, we successfully performed ICA to target the temporal dynamics of face selection in the context of visual face similarity. ICA identified, across subjects, two clusters of independent components (ICS) that reflected the two most relevant face-related event related potential (ERP) components: the N170 and the N250. In line with the fMRI results from Chapter 2, the pattern of activity within the N170 and N250-cluster supported the biased competition model for face selection. 
Due to a very similar stimulation (pair of faces), we expected in the present experiment to find ICs related to face processing, like the N170 and the N250. However, in this case the similarity manipulation between the two faces was related to their semantic information (and not to the physical features). It has been shown that the N170 indexes the structural processing of a face and that it is unaffected by its semantic representation (Bentin S, Allison, T., Puce, A., Perez, E., and McCarthy, G., 1996; Eimer M, 2000, 2000; Herzmann G et al., 2004; Itier RJ and MJ Taylor, 2002; Jemel B et al., 2003; Kaufmann JM et al., 2009; Tanaka JW et al., 2006). Consequently, we did not expect the N170 to be modulated by semantic relatedness neither in the attend-to-face nor in the ignore-faces condition.

On the other hand, based on previous findings, a relatedness effect around 250 ms was predicted. In fact, in a recent review, Galdo Alvarez S et al. (2009) suggested that within the N250 time-window the access to the semantic information related to faces starts to take place. This component was also shown to be the earliest ERP component sensitive to semantic processing (Martin-Loeches M, 2007). The binding between the semantic and visual representation of a face within the $250 \mathrm{~ms}$ time-window was confirmed by several studies that showed the strong sensitivity of the N250 to face familiarity (Kaufmann JM et al., 2009; Schweinberger SR et al., 2004; Tanaka JW et al., 2006). Martin-Loeches M et al. (2001) also suggested that the $\mathrm{N} 250$ might represent the first stage of integration between these two types of information within the fusiform gyrus. This initial process of integration might be significantly hampered by the context, i.e. by presenting next to the target faces a face or object that is semantically related to the target (Hocking J et al., 2010).

Therefore, in line with our fMRI findings in FFA (attend-to-related-face > attend-tounrelated-face), we expected the N250 to show a larger amplitude for the related compared to unrelated faces in the attend-to-face task. In fact, we assumed, as also suggested by Hocking et al. (2010), that the attentional system would enhance the neural activity, here reflected by an increase of the signal amplitude in the N250, when semantic interference makes semantic selection more difficult (attend-to-related-face condition).

In the ignore-faces task, the explicit selection of a face according to specific semantic information was not required. The subjects' attention was focused on the center of the screen where they had to perform a task unrelated to semantic or face processing. Therefore, the integration between a face and the associated profession within the fusiform gyrus was not needed and any interference effect was to be excluded as well as any modulation by relatedness. However, in the preceding fMRI experiment, we observed a context dependent modulation in FFA also in the ignore-faces condition. Due to the low temporal resolution of fMRI we were not able to empirically show whether this effect was indirectly caused by the neural competition we observed in the IFG or rather by semantic competitive interactions occurring locally in FFA. In the latter case we would expect a semantic relatedness effect for the ignore-faces condition already at the N250 time-window. In contrast, in case neural competition was strictly related to the selection process in the IFG no context effects would be expected in EEG at an early stage. 
In later time-windows, we expected that the ICA would identify two other clusters of ICS representing respectively the N300 and N400 ERP components. The N300 has been shown to reflect semantic processing specific to pictorial stimuli and the initial categorization of the objects presented (Barrett SE and MD Rugg, 1990; Eddy M et al., 2006; Ganis G et al., 1996; Hamm JP et al., 2002; Holcomb PJ and WB McPherson, 1994; McPherson WB and PJ Holcomb, 1999; Zhang $Y$ and C Guo, 2008). The N400, mostly distributed parietally, was first reported by Kutas M and SA Hillyard (1980) for sentence processing. This ERP component reflects semantic integration in language production and more recently, it has been associated with a deeper semantic identification of the visual stimuli (Hamm JP et al., 2002; McPherson WB and PJ Holcomb, 1999; Schendan HE and M Kutas, 2002; Sitnikova T et al., 2006; West WC and PJ Holcomb, 2002). A recent EEG experiment showed that the N300 and the $\mathrm{N} 400$ were both sensitive to the semantic content of objects also in the absence of awareness (Eddy $\mathrm{M}$ et al., 2006).

Therefore, we expected that the N300 and the N400 would reflect the automatic activation of the semantic representations of the two faces (ignore-faces condition). In particular, based on the IFG results in the ignore-faces condition (ignore-related-faces > ignoreunrelated-faces) we would expect a similar relatedness effect in this EEG study. We also expected that the two semantic representations (professions from the two faces) would compete with each other. In line with the type of competition we suggested to occur in the IFG and with the specificity for category processing of the N300 (Hamm JP et al., 2002) we expected in the $\mathrm{N} 300$ time-window a competition at the category level (between two different categories).

In the attend-to-faces condition, the absence of any relatedness effect in the fMRIbased IFG activity indicated that during the selection phase, attention was able to fully filter out the distracting face and bias the semantic processing towards the relevant information related to the target face only. This in turn would result in a lack of context effect within either the N300 or the N400 time-window.

\section{Methods}

\subsection{Subjects}

Twenty healthy volunteers ( 15 female, mean age $=25$ years) participated in the experiment. Due to technical problems during the measuring phase one subject was discarded from the final statistical analysis. They were all native Dutch speakers and they had normal or corrected-to-normal vision. After explanation of the procedures, participants signed an informed consent form. Subjects were paid or received credit as compensation for their participation to the experiment. The study was approved by the ethical committee of the faculty. 


\subsection{Stimulus preparation}

The stimuli consisted of two sets of 8 pairs of faces that were respectively semantically related (REL) and unrelated (UNREL) to each other (see Figure 3.a and Stimulus preparation in Chapter 4, for more details on the selection of REL and UNREL stimuli)

\subsection{Procedure in face-profession association phase}

Prior to the EEG measurement, participants needed to associate to each face a specific profession in order to perform the main experiment. This procedure consisted of two phases: a learning phase and a testing phase. A detailed description of the two phases is presented in "Procedure in face-profession association phase" in Chapter 4.

\subsection{Procedure in the main experiment}

The experiment consisted of four runs (Figure 1). Each run consisted of 4 blocks of trials (block-design) and started with a white fixation cross lasting for 3 seconds centred on a blank screen. A single block consisted of 36 trials. Each trial started with the presentation of a white fixation cross of a jittered duration (mean $2500 \mathrm{~ms}$, range 2000-3000 ms) in the centre of a blank screen. The blank condition was followed by a pair of faces presented for $500 \mathrm{~ms}$. The two faces were positioned in opposite hemifields and they were either REL or UNREL to each other at the semantic level (see Stimulus preparation). The single pictures subtended approximately $4.1^{\circ}$ (height) $\times 3.5^{\circ}$ (width) of visual angle and were aligned with the centre of the screen. Between the two faces, two bars $\left(0.2^{\circ}\right.$ in height) were also presented. The width of the bars varied between 1 and 5 pixels and they were placed $0.17^{\circ}$ above and below the centre of the visual screen. The entire visual display covered $4.1^{\circ}$ (height) $\times 7.8^{\circ}$ (width) of visual angle. The display was designed in order to be comparable in size with those used in previous studies on face processing (Gauthier l et al., 2000; Halgren $\mathrm{E}$ et al., 1999; Kanwisher N et al., 1997; Rossion B et al., 2003; Steeves JK et al., 2006; Wojciulik E et al., 1998). Therefore, it was reasonable to assume that FFA integrated the two simultaneously presented faces within each experimental trial (Halgren E et al., 1999; Reinholz J and $S$ Pollmann, 2007). A block of trials ended with a resting condition consisting of a blank screen with a white cross lasting for 2 seconds. A visual instruction was displayed at the beginning of each block for 3 seconds. The block of trials started after the presentation of a white cross on a blank screen ( 2 seconds long). The instruction indicated to the subject to perform either an ignore-faces (IGN) or an attend-to-face (ATT) task (see Figure 3.a and "Procedure in the main experiment" in Chapter 4, for more details on the two tasks). 


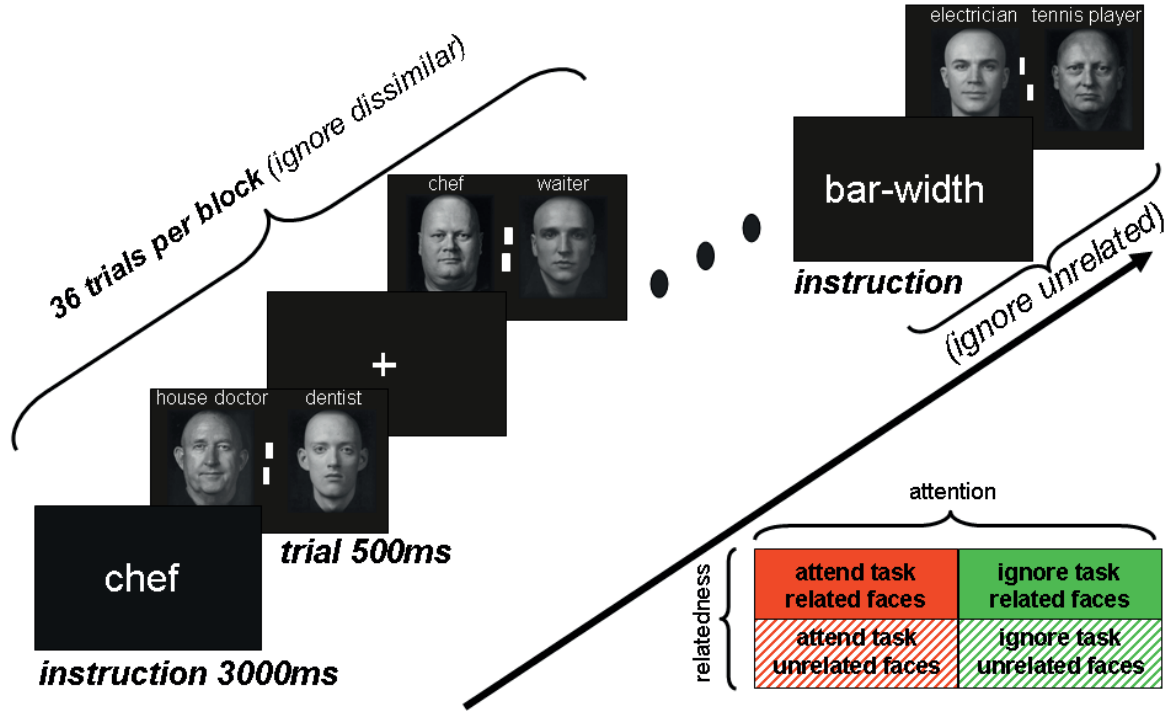

Figure 1. Experimental design. Block structure with examples of two different conditions (attend-to-faces with related display and ignore-faces with unrelated display).

In both tasks, the subject was asked to respond as fast and accurately as possible within a $1500 \mathrm{~ms}$ time-window (comprising the face pair duration - $500 \mathrm{~ms}$ - and 1 second of blank screen). The design was a $2 \times 2$ repeated measures design with factors attention (ATT, IGN) and relatedness (REL, UNREL), leading to 4 conditions (IGN_REL, IGN_UNREL, ATT_REL, ATT_UNREL, see Figure 3.b in Chapter 4). The participants were not made aware of the relatedness manipulation. The order of the blocks was randomised and counterbalanced across participants and runs by means of the Williams design (Williams EJ, 1949). This scheme is a generalized Latin square design, balanced for first-order carryover effects. The stimulus display was identical for both types of task.

After the explanation of the task, and application of the electrodes, participants were comfortably seated in an electrically shielded, sound-attenuated room in front of a computer monitor at a distance of 80 centimeters. The stimuli were delivered using the Presentation 12.2 package (Neurobehavioral Systems, Inc. www.neurobs.com). Participants were instructed to fixate the centre of the screen throughout the experiment and to minimise head and eye-movements for the duration of the experiment. The entire session lasted approximately 40 minutes. 


\subsection{EEG recording}

The electroencephalogram (EEG) was measured using 29 tin electrodes mounted in an elastic cap (QuickCap, Neuroscan Inc. Tm, see "EEG recording" in Chapter 3, for more details).

\subsection{Analysis}

EEG data were epoched with an interval of $900 \mathrm{~ms}$, time-locked to the onset of the presentation of the pair of faces. Epochs included a 100-ms prestimulus baseline correction interval and they were $0.3-30 \mathrm{~Hz}$ bandpass filtered (Butterworth, zero phase, $24 \mathrm{~dB} / \mathrm{oct}$ ). Data were decomposed in independent components (ICS) via ICA. This procedure and the rationale behind it were described in details in "Analysis" in Chapter 3.

\section{Results}

\subsection{Behavioral Results}

We computed error rates (ERs) and correct reaction times (RTs) on the ignore-faces (IGN) and attend-to-face (ATT) task for related (REL) and unrelated (UNREL) condition. Mean ERs and RTs were then submitted to a repeated measures ANOVA with two factors, namely attention and relatedness. For RTs, a significant main effect of attention was observed but no effect of relatedness or interaction. Attention: $(F(1,18)=25.408, p=.000$, mean reaction time $A T T=806 \mathrm{~ms}, \mathrm{SE}=34$, mean IGN $=666 \mathrm{~ms}, \mathrm{SE}=23)$. Relatedness: $(\mathrm{F}(1,18)=1.223, \mathrm{p}=$ .283 , mean REL $=756 \mathrm{~ms}, \mathrm{SE}=34$, mean UNREL $=715 \mathrm{~ms}, \mathrm{SE}=29)$. Interaction: $(F(1,18)$ $=.644, p=.433$ ). Similarly to the RT results, for ERs we did not find any main effects of attention or relatedness or interaction. Attention: $(F(1,18)=2.552, p=.128$, mean ATT $=25 \%$, SE $=3$, mean IGN $=21 \%$, SE = 3). Relatedness: $(F(1,18)=.001, p=.982$, mean $\mathrm{REL}=23 \%, \mathrm{SE}=$ 3 , mean UNREL $=23 \%$, SE $=2)$. Interaction: $(F(1,18)=.111, p=.743)$.

\subsection{ICA clusters}

The ICA cluster analysis revealed 4 clusters of interest namely the N170, N250, N300 and the N400-cluster. The N170-cluster consisted of 12 components from 8 subjects. They were characterized by a negativity peaking around $180 \mathrm{~ms}$. For each IC we computed the average amplitudes around the negative peak (see Analysis). A 2x2 ANOVA performed on these values did not reveal any main effect of attention, relatedness or interaction of factors (Figure 2.a).

The scalp maps of the ICs showed a centro-occipital distribution. These maps were used for source dipole modeling. The N170 has been associated with the first phase of face encoding corresponding anatomically with a face selective region located on the lateral bank of 
the lateral occipital complex (LOC) and known as occipital face area (OFA) (Haxby JV et al., 2000; Schweinberger SR et al., 2007; Schweinberger SR et al., 2002). In order to investigate whether OFA was a realistic source of the N170, we seeded a two-equivalent current dipole into the centro-lateral part of the inferior occipital gyrus (Figure 2.b). The reason we seeded two lateral dipoles instead of one is that, according to the algorithm of ICs extraction, a component with a central distribution reflects two bilateral sources synchronously activated. This source dipole model accounted on average for $92 \%$ of the observed variance.

a)

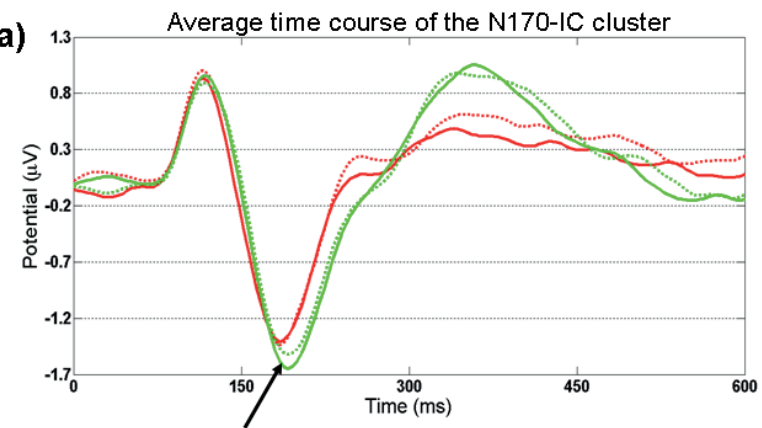

b)

ICs contribution over the scalp

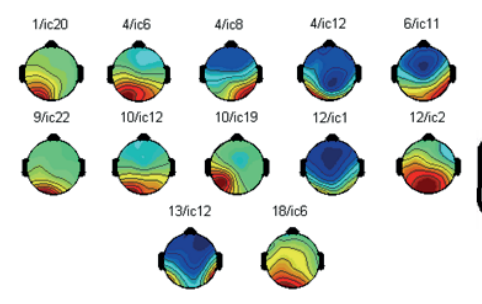

average distribution

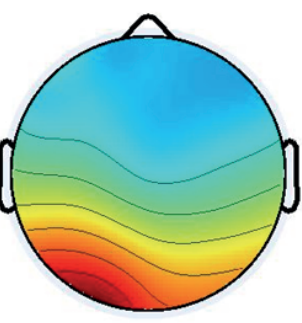

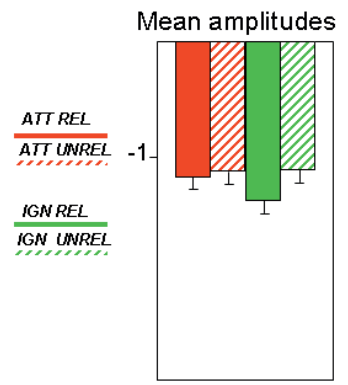

$\mathrm{N} 170$

dipole source modeling

$92 \%$

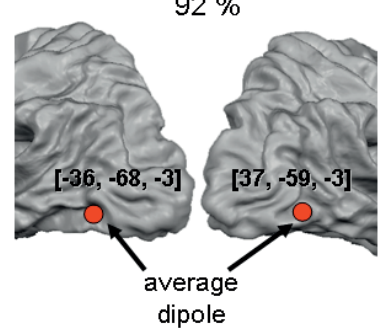

Figure 2. Result of the ICA analysis of the EEG data: N170. (a) Left: Average of the ERPs associated with the ICS of the N170-cluster. Right: results of the 2×2 ANOVA analysis on the mean-ERP-amplitudes of the ICs in a window of $60 \mathrm{~ms}$ around $180 \mathrm{~ms}$ (average peak). (b) Left: scalp maps associated to the ICs of the N170-cluster. Center: Average of the ICS spatial distribution. Right: location of the two-dipoles-model used to fit the ICs scalp maps.

The clustering procedure revealed another face-specific cluster that consisted of 13 components (from 11 subjects) identified by a negative deflection around $240 \mathrm{~ms}$. For its characteristics we defined this cluster as the N250-cluster. For the components in this cluster we observed a significant interaction of factors attention and relatedness (Figure 3.a). Attention: $(\mathrm{F}(1,10)=.024, \mathrm{p}=.880$, mean amplitude $\mathrm{ATT}=-1.316, \mathrm{SE}=.402, \mathrm{IGN}=-1.340, \mathrm{SE}=.388)$. Relatedness: $(F(1,10)=.050, p=.828$, mean amplitude $R E L=-1.333, S E=.393$, UNREL $=-$ $1.323, \mathrm{SE}=.383)$. Interaction: $(\mathrm{F}(1,10)=8.375, \mathrm{p}=.016)$. 
a)

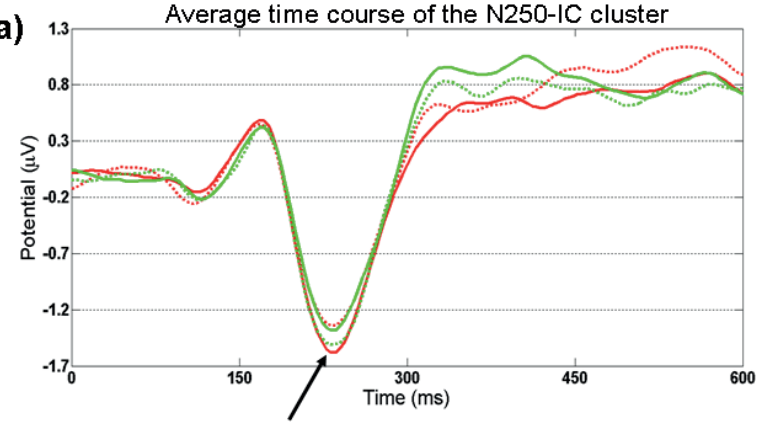

b)

ICs contribution over the scalp

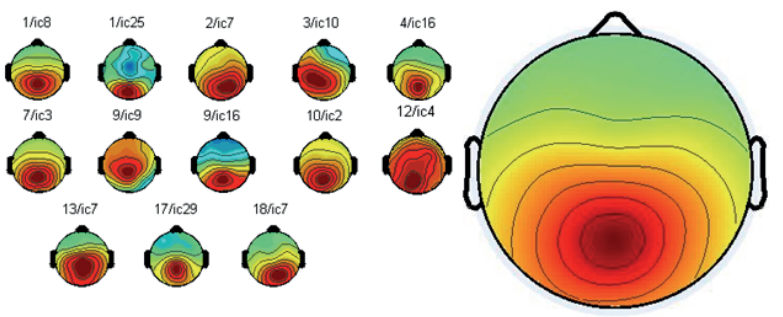

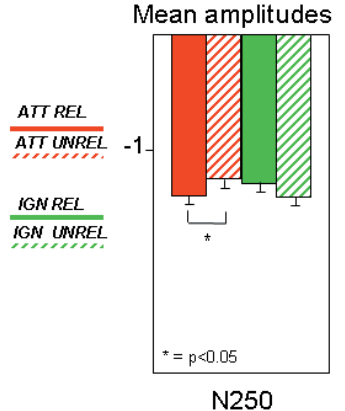

dipole source modeling

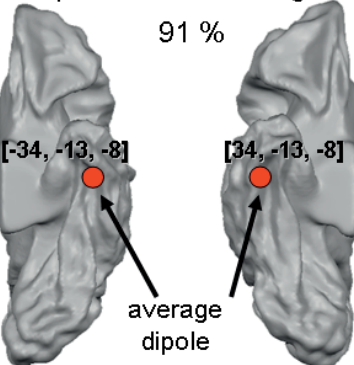

Figure 3. Result of the ICA analysis of the EEG data: N250. (a) Left: Average of the ERPs associated with the ICS of the N250-cluster. Right: results of the 2×2 ANOVA analysis on the mean-ERP-amplitudes of the ICs in a window of $60 \mathrm{~ms}$ around $230 \mathrm{~ms}$ (average peak). (b) Left: scalp maps associated to the ICs of the N250-cluster. Center: Average of the ICs spatial distribution. Right: location of the two-dipoles-model used to fit the ICs scalp maps.

The interaction effect was further investigated by comparing related vs. unrelated condition within the attend-to-face and the ignore-faces condition separately. The pairwise comparisons showed no difference in the ignore-faces condition, but a significant effect of relatedness within the attend-to-face condition (ATT_REL > ATT_UNREL: $F=6.07, p=.033$; IGN_REL vs. IGN_UNREL: $F=3.03 p=.112$ ). The same $2 \times 2$ ANOVA was performed on the backprojections of the ICs onto the scalp (original space). This analysis, in line with the previous one, revealed an interaction effect $(F(1,10)=11.768, p=.006)$. This interaction reflected, once again, no relatedness effect within the ignore-faces condition but a significant difference between attend-to-related-face and attend-to-unrelated-face (ATT_REL > ATT_UNREL: $\mathrm{F}=10.33, \mathrm{p}=.009 ;$ IGN_REL vs. IGN_UNREL: $\mathrm{F}=2.27, \mathrm{p}=.163$ ).

The IC scalp maps were characterized by a posterior-parietal distribution. Several studies suggested based on source modeling that the N250 is generated in FFA (Schweinberger SR et al., 2004; Schweinberger SR et al., 2007; Schweinberger SR et al., 2002). Therefore, similarly to the seeding procedure we use for the N170, we modeled the scalp maps of the N250-cluster with two symmetrical dipoles located bilaterally in the anterior part of the 
fusiform gyrus (Figure 3.b). These dipole models located within FFA accounted on average for $91 \%$ of the total variance.

The N300-cluster consisted of 15 components from 10 subjects. The scalp maps of the N300-ICs had maxima over the centro-frontal electrodes. They were also characterized by a large negativity around $270 \mathrm{~ms}$. A $2 \times 2$ ANOVA on the ICs mean amplitudes revealed no main effect of attention or relatedness but a significant interaction effect (Figure 4.a). Attention: $(\mathrm{F}(1,9)=.270, \mathrm{p}=.616$, mean amplitude ATT $=-1.606, \mathrm{SE}=.697, \mathrm{IGN}=-1.880, \mathrm{SE}=1.120)$. Relatedness: $(F(1,9)=.559, p=.474$, mean amplitude REL $=-1.726, \mathrm{SE}=.896, \mathrm{UNREL}=-$ 1.760, SE = .894). Interaction: $(F(1,9)=8.654, p=.016)$. This interaction effect reflected a significant difference between related and unrelated condition within the attend-to-face and ignore-faces task (ATT_REL < ATT_UNREL: $F=5.57, p=.043$; IGN_REL > IGN_UNREL: $F=8.33$ $p=.018)$. We found the same pattern of results when we back-projected the ICs of the N300-cluster onto the scalp. In fact, a 2×2 ANOVA revealed an interaction attention and relatedness $(F(1,9)=6.201, p=.034)$. The pairwise comparisons between related and unrelated condition performed separately for both tasks confirmed a relatedness modulation of the N300 component in both tasks (ATT_REL < ATT_UNREL: F = 5.21, p = .048; IGN_REL > IGN_UNREL: $\mathrm{F}=5.11 \mathrm{p}=.050)$.

a)

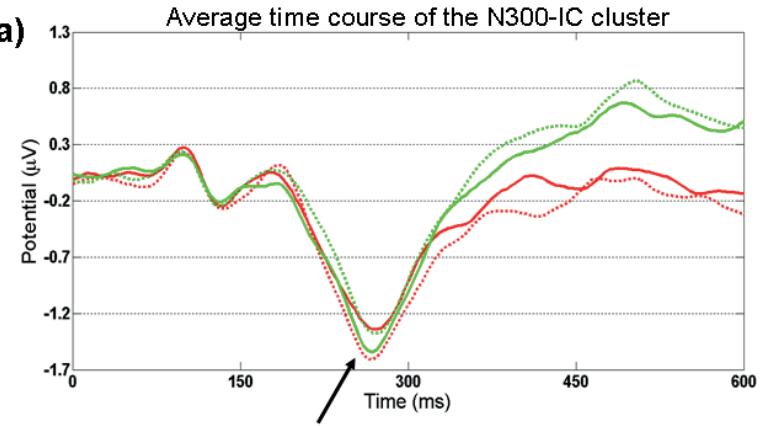

b)

ICs contribution over the scalp

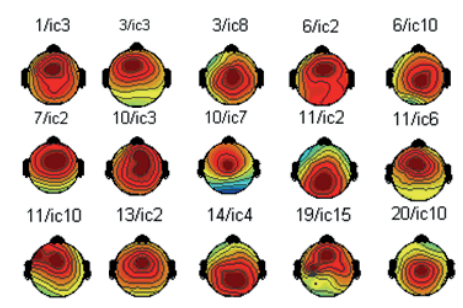

average distribution

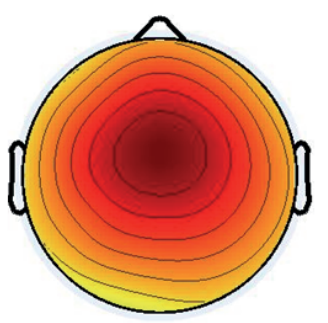

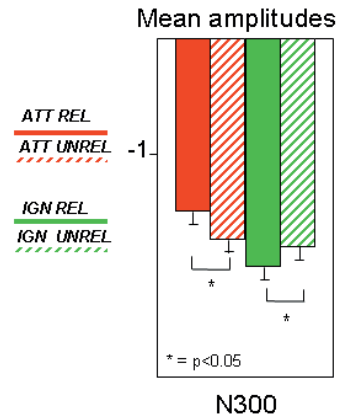

dipole source modeling

$92 \%$

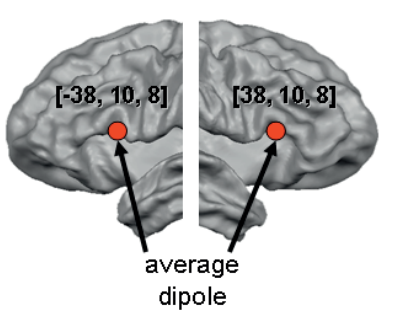

Figure 4. Result of the ICA analysis of the EEG data: N300. (a) Left: Average of the ERPs associated with the ICs of the N300-cluster. Right: results of the 2×2 ANOVA analysis on the mean-ERP-amplitudes of the ICs in a window of $60 \mathrm{~ms}$ around 270 ms. (b) Left: scalp maps associated to the ICs of the N300-cluster. Center: Average of the ICs spatial distribution. Right: location of the two-dipoles-model used to fit the ICs scalp maps. 
Based on the frontal distribution of the N300-ICs and on the key-role of the IFG in the fMRI study (Chapter 4), we tested whether this region was a plausible neural generator of the N300 for the present experiment. In particular, we used the same seeding procedure performed for the N170 and the N250. We observed that a two-dipole model in IFG explained on average the $92 \%$ of the total variance.

The N400-cluster consisted of 13 components from 11 subjects. In the temporal domain, the ICs shared a negative deflection at about $400 \mathrm{~ms}$. The statistical analysis on the mean amplitudes around this peak did not reveal any main effect of attention, relatedness or interaction of factors (Figure 5.a). The N400-ICs were maximally distributed in the midline over the posterior electrodes.
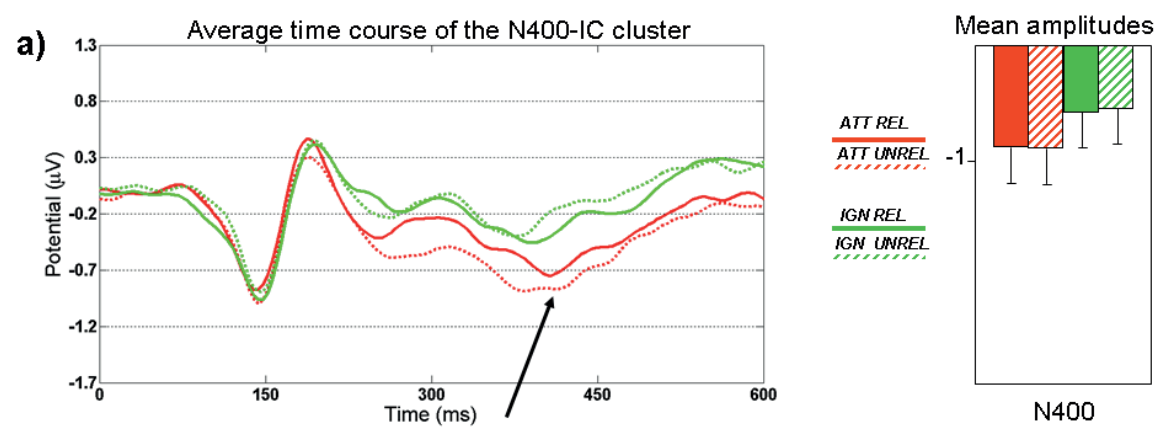

b)

ICs contribution over the scalp

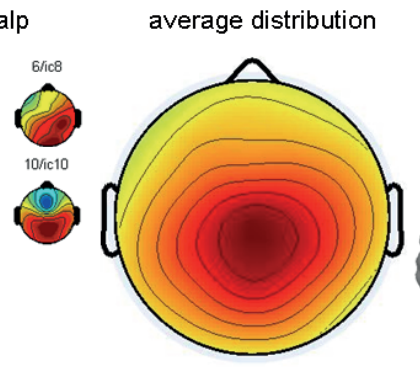

dipole source modeling

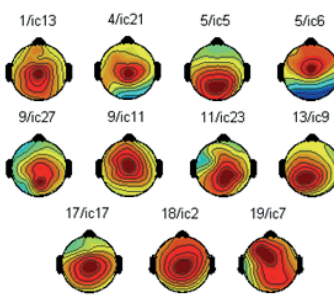

$90 \%$

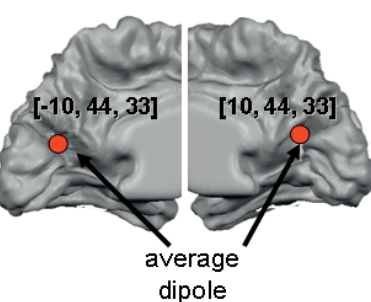

Figure 5. Result of the ICA analysis of the EEG data: N400. (a) Left: Average of the ERPs associated with the ICs of the N400-cluster. Right: results of the 2x2 ANOVA analysis on the mean-ERP-amplitudes of the ICs in a window of $60 \mathrm{~ms}$ around 400 ms. (b) Left: scalp maps associated to the ICs of the N400-cluster. Center: Average of the ICs spatial distribution. Right: location of the two-dipoles-model used to fit the ICs scalp maps.

In contrast to the clusters analyzed so far, we did not have a clear expectation on the source location of the N400. In fact, the posterior distribution excluded the possibility to seed a dipole into the IFG, as for the N300. Therefore, we did not proceed with the seeding of dipoles in a specific area. Instead, we computed for each IC an equivalent current dipole (a one or two dipole model) identified by a projection on the scalp that best-fitted the observed scalp topography of that component. The best fitting dipoles were mainly located within two 
neighboring regions corresponding to the superior parietal lobe and the precuneus. On average these dipoles accounted for $90 \%$ of the total variance (Figure 5.b).

In order to test whether the N250 and N300 clusters, the most relevant for the present study, were representative of the same ERP components on the scalp, we computed the contribution of the IC-back-projections to the grand average ERP in two corresponding timewindows: $180-240 \mathrm{~ms}$ (N250) and 210-270 ms (N300). In the 180-240 ms time-window we observed that the $\mathrm{N} 250$ was the cluster that contributed the most to the negative EEG signal on the scalp for all conditions (ATT_REL $=30.33 \%$, ATT_UNREL $=25.71 \%$, IGN_REL $=31.25$ $\%$, IGN_UNREL $=30.64 \%$ ). The same held for the N300 cluster within the $210-270$ ms timewindow conditions (ATT_REL $=24.81 \%$, ATT_UNREL $=21.54 \%$, IGN_REL $=24.01 \%$, IGN_UNREL $=19.85 \%$ ).

\section{Discussion}

In the present EEG study we investigated the temporal characteristics of semantic selection in the context of face processing. We simultaneously presented two faces that were either semantically related (same category-professions) or unrelated to each other (different category-professions). The participants were asked to attend to one of the faces (attend-to-face condition) or ignore both of them (ignore-faces condition). In Chapter 4, we reported the data from an identical experiment using fMRI. We disentangled, to our knowledge for the first time, the two main processes occurring during the semantic selection of a face in the presence of a distractor: the competitive interaction between the semantic representations related to the two faces (ignore-faces task) and the selection of the relevant face (attend-toface task). This study revealed a functional dissociation between two relevant areas involved in semantic selection: FFA and IFG. In particular, we observed in FFA a relatedness effect (related > unrelated) for both ignored and attended faces. In the IFG, the relatedness effects were present for ignored cases only. This pattern could be explained by a multiple-stage model where in addition to the competition (access) and selection phases modeled by Levelt WJM et al. (1991), the occurrence of an interference mechanism in FFA was proposed. We suggested that in a first stage the visual features and semantic information (professions) of the two faces are integrated within FFA. In line with Hocking J et al.(2010), when the relevant face had to be selected, this process appeared to be more demanding when the distracting face was semantically related to the target. In a later phase of semantic selection, the associated visual and semantic information is passed on from FFA to IFG, were selection takes place. At this stage the two professions were encoded also when ignored and they competed for representation at the categorical level (Kan IP and SL Thompson-Schill, 2004). Selection was finally accomplished in the IFG as we found that attention to one face was able to filter the distracting face in favor of the semantic processing of the relevant profession only. 
The present EEG experiment aimed to further investigate these temporal assumptions. More specifically, we tested whether face selective ERP components (the N170 and the N250) reflected a semantic interference effect that is typically observed in the early stage of semantic selection when visual and semantic information are integrated. We also investigated the occurrence of competitive interactions at the level of the two most relevant ERP components for semantic selection and integration: the N300 and N400. Finally, we tested, in the same time-windows, whether and how selection was accomplished

The data were analyzed with ICA. This procedure identified four clusters of interest: the $\mathrm{N} 170$, the N250, the N300 and the N400. The labels given to each of these clusters were defined according to the properties of the related ICs. The time course and the spatial distribution of the N170-ICs, for example, closely resembled the temporal and spatial characteristics of the N170 ERP component. In the following paragraphs we will comment the results related to each of these clusters.

\subsection{The N170 cluster}

The most relevant feature of the N170-ICs was a negative deflection peaking at about 170 ms. The associated scalp maps revealed that the contribution of the ICs was maximum over the most posterior electrodes, which corresponded most likely to the occipital lobe. In line with previous findings, the source dipole modeling confirmed the occipital origin of the N170 within the inferior occipital gyrus (Haxby JV et al., 2000; Schweinberger SR et al., 2007; Schweinberger SR et al., 2002).

The statistical analysis did not reveal any effect of attention or relatedness for the N170-ICs. The N170 ERP component has been associated with the early phase of face processing related to the structural encoding and the feature-based analysis of a face (Bruce $\mathrm{V}$ and A Young, 1986; Haxby JV et al., 2000). In the present experiment, we carefully controlled for any difference in physical similarity such that the two faces in the displayed pair were "equally dissimilar" in all conditions (see Stimulus preparation). Hence, the absence of any effect in the N170 was in line with the role of this component in face processing and with the rationale of the design that was focused on a semantic context manipulation. Therefore, we can conclude that the semantic context did not play a systematic role during information processing in the N170 time-window.

\subsection{The N250 cluster}

The ICs in this cluster presented a clear negativity around $240 \mathrm{~ms}$. The ICs-topographies were characterized by a posterior-parietal distribution. A two-equivalent dipole model was seeded within FFA in order to test whether this area was a plausible neural generator for the N250. The high amount of variance explained by this model confirmed this assumption, and replicates the results by Schweinberger SR et al. (2004; 2007; 2002). 
The 2x2 ANOVA on the N250-ICs showed an interaction of factors attention and relatedness. A planned pairwise comparison, computed separately for the two tasks, revealed a significant relatedness modulation for the attend-to-face and not for the ignore-faces condition. In the attend-to-face condition, we found that semantically related faces elicited a higher signal than the unrelated faces. In line with the same relatedness effect we found in the preceding fMRI experiment in FFA, this result suggested that the semantically related distractor interferes with the visual-semantic association mechanism in the $250 \mathrm{~ms}$ time-window. In fact, according to the pre-lexical account of the semantic interference effect, FFA represents the first stage where semantic and perceptual information are integrated. This in turn caused a larger semantic interference in the related condition which is solved by the attentional system within FFA (Hocking J et al.(2010).

Unlike the $\mathrm{fMRI}$ results in FFA, the $\mathrm{N} 250$ did not show a relatedness modulation within the ignore-faces condition. This result is in line with the suggested functional role of this ERP component. In particular, the N250 is involved in the holistic configuration of a face as well as the first stage of its semantic encoding. But in the ignore-faces task, no explicit integration of a face and the associated profession was necessary. In addition, the N250 does not represent the semantic processing per se. Hence, we also would not expect the automatic extraction of the two professions and their eventual competition. At this early stage, FFA probably just passes the information through towards the frontal lobe. Therefore, the fMRI based FFA modulation we observed in Chapter 4 (ignore-related-faces > ignore-unrelated-faces) probably occurred due to the top-down influence of a later IFG activity.

\subsection{The N300 cluster}

The N300-ICs peaked at about $270 \mathrm{~ms}$ in correspondence of a negative deflection. The scalp maps associated with the N300-ICs showed a focal distribution over the fronto-central electrodes. In turn, the successful fit of these maps with those generated by a two-dipole-model in IFG identified this region as the neural source of the N300. The pattern of activation in the N300 was similar to the results we found in the IFG in fMRI. In fact, as in the IFG, we observed for the $\mathrm{N} 300$ a significant interaction between the two factors attention and relatedness. A pairwise comparison performed for the ignore-faces condition revealed a higher EEG signal for the related vs. the unrelated faces. The N300 has been shown to reflect the initial stage of semantic processing, which is mostly related to the categorization of pictorial stimuli (Barrett SE and MD Rugg, 1990; Ganis G et al., 1996; Hamm JP et al., 2002; Holcomb PJ and WB McPherson, 1994; McPherson WB and PJ Holcomb, 1999; Zhang Y and C Guo, 2008). Therefore, the relatedness effect in the N300 suggested that faces were semantically encoded at the category level (e.g. physician, sport-men) even when ignored, as also showed by Eddy $\mathrm{M}$ et al. (2006). The same modulation effect was observed in $\mathrm{fMRI}$ for unattended faces in the IFG. The convergence between the N300 and the IFG with respect to the semantic modulation in the ignore-faces task suggested that around $300 \mathrm{~ms}$ post stimuli onset 
professions categories were automatically extracted in the IFG from the two unattended faces. In addition, if we assume that this difference reflects semantic competition and that larger amount of competitive interactions results in a lower EEG signal, the relatedness effect in the $\mathrm{N} 300$ time-window suggested that two unrelated faces competed with each other more than related faces. Therefore, in line with the finding in the IFG, the lower ERP amplitude in the N300 might reflect a category competition effect. In fact, in the ignoreunrelated-faces, the coactivation of two different category-nodes could give rise to the activation of the semantic nodes connected to each category. This spread of activation between nodes in the semantic network would lead to a stronger competition due to a higher amount of inhibitory connections. Therefore, competitive interactions would be larger for between-categories (unrelated faces) compared to within-category condition (related faces).

At first sight this result might seem in contrast with psycholinguistic theories on semantic competition (Oppenheim GM et al., ; Vigliocco G et al., 2002) as these models would predict stronger competition when two items belonged to the same category, reflecting, in turn, a lower EEG signal for related faces. However, most of these theories were tested when selection was in place (object naming), which can be considered as an "attend" task where attentional mechanisms are involved. In contrary, the present ignore-faces condition was introduced to test the interaction of two semantically related and unrelated faces in the absence of attention.

The pairwise test within the attend-to-face task also showed a significant difference between the related and unrelated-faces condition but with the opposite pattern compared to the ignore-faces task (attend-to-unrelated-faces > attend-to-related-faces). This relatedness modulation suggested that semantic selection was affected by the distracting face at the level of the N300. According to Oppenheim GM et al. (2010) and Vigliocco G et al. (2002), lateral inhibition, during semantic selection, suppresses the processing of the target in a larger extent when two items share similar features compared to when they are semantically unrelated. The presence of the context effect in addition to the lower signal in the related case indicated that attention did not fully bias the selection yet. It rather seemed that several alternative candidates (here two professions) are still competing with each other for selection.

In contrast to this modulation in the N300, the fMRI experiment did not reveal any relatedness effect in the attend-to-face condition. The $\mathrm{fMRI}$ results suggested that attention to one face solved the competition that we observed during the ignore-faces task when both semantic representations of faces interacted with each other (Grindrod CM et al., 2008; Kan IP and SL Thompson-Schill, 2004). However, due to the sluggishness of the hemodynamic response, the $\mathrm{fMRI}$ signal represents the temporal integration of a series of neural events occurring in a time-window of few seconds. The relatedness modulation we observed at the level of the N300 might reflect one of those events and the input from FFA into IFG within the N250 time-window (attend-to-related-face > attend-to-unrelated-face, opposite pattern compared to the N300) might represent an earlier stage of selection. Averaged over time, 
these two effects might cancel each other out in $\mathrm{FMRI}$, resulting in a lack of relatedness modulation at the level of the IFG. In contrast, the EEG was able to disentangle the two different processes over time.

In sum, the bias effect we hypothesized based on the fMRI activation in the IFG during the attend-to-face task might be originated from two different mechanisms. The first, occurring at around $250 \mathrm{~ms}$, represented an attentional mechanism to solve the semantic interference in FFA and the other, taking place in the IFG at about $300 \mathrm{~ms}$, reflected an inhibitory effect from the related face distractor.

\subsection{The N400 cluster}

The ICA algorithm also identified a N400-cluster of ICs characterized by a wide negativity peaking at about $400 \mathrm{~ms}$. In this cluster we did not observe any significant effect of attention, relatedness or interaction. The ICs scalp maps had a centro-parietal distribution. N400ICs were well modeled by two symmetrical dipoles located within the superior parietal lobe and precuneus. Also in a recent study on related and unrelated word-picture pairs, the N400 has been localized within the precuneus (Zhang Y and C Guo, 2008). The precunes has been associated with memory retrieval, mostly when visual imagery is involved (Dorfel D et al., 2009; Fletcher PC et al., 1995). In our study and in particular in the attend-to-face task, subjects might have used strategies that relied on visual imagery to retrieve the association between the face presented and a specific profession. The lack of a relatedness modulation in the attend-to-face task suggested that the pairing face-profession was equally successful in both conditions and that the distracting face did not influence this process within the N400 time-window. We can conclude that at this stage of semantic processing attention acted to solve any inhibitory effect from the context, which resulted in the ultimate selection of the target-face. Finally, the absence of any effect in the ignore-faces suggested that competition among different semantic representations only occurred during conceptual coactivation (in the IFG) and not during upgrading retrieved information with memory representations (in the precuneus).

In summary, the combination of the previous $\mathrm{FMRI}$ results and the present EEG data suggested a functional segregation of FFA and IFG as key regions in the semantic selection of faces. The data supported the idea that semantic selection took place in three distinct timewindows. In the early stage, within the FFA and in the N250 time-window, the initial integration of a face and the associated profession is a context sensitive process. In fact, the distracting face hampered this mechanism and interference was stronger for related faces compared to unrelated face. Attention solved this inhibiting effect of the distractor by enhancing the activity in the most demanding case (attend-to-related-face)

In a later time-window, around 300 ms post stimulus onset, the semantic representations were coactivated in the IFG also when both faces were unattended and they interacted 
in a competitive manner. When active selection was needed (attend-to-face task), the EEG results suggested that the distracting face was still processed to some extent and that it affected the processing of the relevant face (Kan IP and SL Thompson-Schill, 2004). Around $400 \mathrm{~ms}$ any context effect was cancelled out. In this time-window attentional mechanism might eventually have solved the preceding interference effect which in turn resulted in an accurate and efficient selection of the target.

\section{Acknowledgments}

The authors wish to thank Job van den Hurk for helpful discussions. This project was supported by Maastricht University (HRM initiative Mobilizing Minds: "Females into higher positions") to B.M. Jansma. 

General discussion 
In the present thesis we investigated the neural correlates of face selection when a target face was presented in the context of another face. We described and compared the mechanism of selection when a face had to be selected with regard to some visual properties or to specific semantic information. Visual and semantic selection was investigated in separate experiments but with identical designs. High spatial (fMRI) and high temporal resolution (EEG) techniques were used to study temporal aspects of face selection within the brain areas of interest.

Face selection was first explored in the visual domain by using fMRI and EEG. In particular, we tested whether face selection works in line with competitive models of selection. Among those, the biased competition model states that the selection of a specific item in a cluster of other objects occurs in two distinct phases. First, in a pre-selective stage, when none of the objects is yet attended to, the two items compete with each other to be represented in the cortex. Secondly, during the selection phase, attention to the relevant item (in a specific location and/or characterized by a specific visual feature) biases the neural competition in favor of the target only. Within this framework attention is not considered anymore as a spotlight that enhances the neural activity related to the attended item. It rather represents the mechanism needed to resolve the competitive interaction between the target and the distractor when they are simultaneously presented. In the face processing domain, the most natural way to test this model was to simultaneously present two faces and design two tasks that allowed us to investigate separately the two phases of face selection modeled by $\mathrm{BC}$. In line with this idea, participants were asked to perform an ignore-faces task where they ignored both faces and an attend-to-face condition where they had to attend to one of them while performing a match-to-sample task. The ignore-faces condition was used to investigate whether the two faces were actually encoded to a certain extent though ignored. We also expected that the simultaneous processing of the two faces would lead to neural competition. In order to test this hypothesis, the two faces presented were similar or dissimilar at the perceptual level. A modulation by similarity would support the simultaneous processing of the two faces as well as the occurrence of competitive interactions. The face similarity manipulation was also employed in the attend-to-face task. In this case we expected attention to bias the face processing towards the target only and in turn to cancel out any context effect of the distracting face. With $\mathrm{fMRI}$ we restricted our investigation to face selective areas where we expected both competition and selection to occur. However, due to the low temporal resolution of $\mathrm{fMRI}$, this technique could not be used to track the temporal dynamics of these mechanisms. For this purpose a similar experimental design was carried out in an EEG experiment. The EEG data were analyzed with ICA.

Furthermore, we used both an $\mathrm{FMRI}$ and an EEG experiment to investigate the underlying neural mechanism of face selection in the semantic domain when a distracting face was contextually presented. As in the two previous studies, we simultaneously presented two faces. In this case, besides its perceptual properties, each face was characterized by additional information, consisting of a specific profession. As in the visual domain, semantic 
selection might also be modeled in two stages: a competition and a selection phase. In fact, prior to selection when the two face-professions have to be accessed, they have the same probability to be represented. Consequently this leads to competitive interaction between the two semantic representations. Attention to one face might bias this competition in favor of the semantic processing of the target face. In order to test this hypothesis the experimental designs included an ignore-faces and attend-to-face condition. The former task was similar to the ignore-faces task, as described above, and it was used to explore whether semantic information from both faces were processed and competed with each other. In the attend-to-face task, participants shifted their attention to one face on which they performed a match-to-profession task. This condition was necessary to test whether attention was able to filter out the irrelevant information. In both tasks, faces were paired in such a way that professions could belong either to the same or different profession-categories (respectively related and unrelated condition). In line with the rationale of the visual similarity manipulation, a semantic relatedness effect in the ignore-faces task would reflect the automatic encoding and competition of the professions, associated with the two faces. In contrast, we expected a lack of relatedness modulation in the attend-to-face condition, as attention to the target should bias the competition and only relevant information was to be processed. The fMRI experiment allowed us to investigate the competitive models of selection in the semantic domain in regions known to play a central role in semantic selection. We also analyzed the pattern of activation in FFA in order to investigate the role of this region when face processing is involved, but when selection is not directly related to the perceptual analysis of the target face. In fact, we expected that in order to select the semantic information associated with the relevant face the system would, first, need to analyze the visual features of the target face and, then, integrate these features with the appropriate profession. We identified the FFA as a possible candidate responsible for this integrative mechanism. EEG in combination with ICA was used to test the timing of all the stages involved in the semantic selection of a face in context: the perceptual analysis of the face, the integration of its semantic and visual information, competition between target and distractor and ultimately the selection of the target.

\section{Visual selection of a face}

The findings in the $\mathrm{FMRI}$ and EEG experiments related to the selection of a face in the context of a distractor supported the BC model of selection. In particular, in the fMRI study, the analysis restricted to FFA revealed that this region was modulated by the similarity manipulation between the two faces when they were simultaneously presented and ignored (ignore-faces task). In addition, the fMRI signal was smaller when the faces were similar to each other compared to dissimilar faces. The same pattern of activation was observed, in the whole brain analysis, within the OFA. We interpreted these results in line with the competi- 
tion effect between the two faces hypothesized in the $B C$ account when two items were simultaneously processed. First, the similarity modulation both in FFA and OFA suggested that the $\mathrm{fMRI}$ signal was sensitive to the similarity level between the two faces which in turn indicated that they were both processed. Secondly, the occurrence of competitive interactions between faces was supported by the lower fMRI signal in the similar compared to the dissimilar case. In fact, if we assume that dissimilar faces are encoded by distinct subpopulation of neurons and similar faces are represented by neighboring (or overlapping) neural populations, we would expect a larger amount of competition in the ignore-similar-faces condition. In the attend-to-face condition, both FFA and OFA did not show any similarity effect. This result suggested that only the target face was encoded whereas the influence of the other face was filtered out. In terms of the BC model, attention biased the competition that occurred in a pre-selective stage (ignore-faces task), towards the processing of the selected face. The ICA-based EEG data revealed two face related ERP components: the N170 and the N250. In line with the FMRI results in FFA and OFA, we observed that the N250 was modulated by face similarity in the ignore-faces condition but not in the attend-to-face condition. In addition, the direction of the similarity effect fitted the pattern we observed in FFA and OFA. However, the N170 was not modulated by the similarity manipulation neither in the ignore-faces nor in the attend-to-face condition, suggesting that the pattern of $\mathrm{FMRI}$ activity in OFA was related to top-down modulation. Overall, the combination of results from the $\mathrm{FMRI}$ and EEG studies suggested that the selection mechanism of a face in the presence of a distracting face works according to the BC model within FFA in the $250 \mathrm{~ms}$ time-window. In particular, when the two faces are simultaneously presented and ignored (pre-selective access phase) they are both encoded and they both competed for representation. When the relevant face has to be selected, attention biased this competitive interaction in favor of the target in a $250 \mathrm{~ms}$ time-window.

\section{Semantic selection of a face}

Face selection was further investigated in the semantic domain with EEG and fMRI. The FMRI experiment aimed to test whether changing the relevance of the target face from its visual features to some semantic property (profession) would result in a spatial shift of the regions involved in the selection mechanism. We found in the IFG a modulation by semantic relatedness in the ignore-faces and not in the attend-to-face condition. This result suggested that when none of the faces and associated professions was relevant for behavior (ignored-faces task) they were still processed to a certain extent in the IFG. The lack of semantic relatedness effect in the IFG in the attend-to-face task indicated that attention to the profession of the target face filtered out the semantic representation of the other face. In line with the competitive models of selection and with the central role of the IFG in semantic processing, we suggested that the semantic selection of a face in a context occurred within the IFG as 
follows. In a pre-selective stage the semantic representation, which are associated with the two faces, are encoded in the IFG and compete with each other. Attention resolved this competition by processing only the profession of the relevant face. In the same experiment a modulation by semantic relatedness within the attend-to-face task was also observed in FFA. We interpreted this result as a semantic interference effect from the distracting face during the integration of the visual and semantic information related to the target face. In the EEG experiment we found the same modulation at the level of the N250 component and only in the attend-to-face task. In a later time-window around $300 \mathrm{~ms}$ post stimulus onset we observed a semantic relatedness effect within the ignore-faces condition, which was similar to the modulation in the IFG. In the same time-window a significant difference between related and unrelated faces was found in the attend-to-face condition, but with an opposite pattern. Finally, the N400 ERP component did not show any modulation neither in the ignore-faces condition and most importantly nor in the attend-to-face task. These results allowed us to draw the timeline of the neural events we observed in fMRI. In the pre-selective stage, the two professions associated with the two faces were automatically extracted and competed to be represented in a $300 \mathrm{~ms}$ time-window. When the profession associated to one of the two faces needed to be selected (selection phase) three different time-windows were identified. In an initial stage, around 250 ms post stimulus onset, attention served to accomplish the integrative process of visual and semantic features related to the target face occurring in FFA. In a later stage (300 ms time-window), attention was not yet able to resolve the competition between the two semantic representations in the IFG. In a final stage, the semantic selection of the target face took place in a $400 \mathrm{~ms}$ time-window where attention to the relevant face was able to reduce and ultimately remove the influence of the irrelevant information from the distracting face.

In conclusion, when a face is presented in the context of another face and it has to be selected according to its visual features, selection occurred in FFA in a 250 time-window. However, when the relevant information of the target face is related to its semantic representation, selection is accomplished in the IFG in later time-windows (300 ms and $400 \mathrm{~ms}$ post stimulus onset). FFA in this case is necessary for an initial phase of selection when the semantic and visual information of the target face has to be integrated. 



\section{References}

Barrett SE, Rugg MD (1990) Event-related potentials and the semantic matching of pictures. Brain Cogn 14: 201-212.

Beck DM, Kastner S (2005) Stimulus context modulates competition in human extrastriate cortex. Nat Neurosci 8: 1110-1116.

Beck DM, Kastner S (2007) Stimulus similarity modulates competitive interactions in human visual cortex. J Vis 7: 19 11-12.

Bentin S, Allison, T., Puce, A., Perez, E., and McCarthy, G. (1996) Electrophysiological studies of face perception in humans. J Cogn Neurosci 551-565.

Bles M, Jansma BM (2008) Phonological processing of ignored distractor pictures, an fMRI investigation. BMC Neurosci 9: 20.

Bles M, Schwarzbach J, De Weerd P, Goebel R, Jansma BM (2006) Receptive field size-dependent attention effects in simultaneously presented stimulus displays. Neuroimage 30: 506-511.

Boksem MA, Meijman TF, Lorist MM (2005) Effects of mental fatigue on attention: an ERP study. Brain Res Cogn Brain Res 25: 107-116.

Bookheimer S (2002) Functional MRI of language: new approaches to understanding the cortical organization of semantic processing. Annu Rev Neurosci 25: 151-188.

Brefczynski JA, DeYoe EA (1999) A physiological correlate of the 'spotlight' of visual attention. Nat Neurosci 2: 370-374.

Broadbent D (1958) Perception and Communication Elmsford, New York: Pergamon.

Brown MR, Goltz HC, Vilis T, Ford KA, Everling S (2006) Inhibition and generation of saccades: rapid event-related fMRI of prosaccades, antisaccades, and nogo trials. Neuroimage 33: 644-659.

Bruce V, Young A (1986) Understanding face recognition. Br J Psychol 77 ( Pt 3): 305-327.

Carrasco M, Ling S, Read S (2004) Attention alters appearance. Nat Neurosci 7: 308-313.

Chelazzi L, Duncan J, Miller EK, Desimone R (1998) Responses of neurons in inferior temporal cortex during memory-guided visual search. J Neurophysiol 80: 2918-2940.

Chelazzi L, Miller EK, Duncan J, Desimone R (2001) Responses of neurons in macaque area V4 during memory-guided visual search. Cereb Cortex 11: 761-772.

Corbetta M, Akbudak E, Conturo TE, Snyder AZ, Ollinger JM, Drury HA, Linenweber MR, Petersen SE, Raichle ME, Van Essen DC, Shulman GL (1998) A common network of functional areas for attention and eye movements. Neuron 21: 761-773.

Corbetta M, Miezin FM, Shulman GL, Petersen SE (1993) A PET study of visuospatial attention. J Neurosci 13: $1202-1226$.

Damian MF, Vigliocco G, Levelt WJ (2001) Effects of semantic context in the naming of pictures and words. Cognition 81: B77-86. 
de Zubicaray G, McMahon K, Eastburn M, Pringle A, Lorenz L (2006) Classic identity negative priming involves accessing semantic representations in the left anterior temporal cortex. Neuroimage 33: 383-390.

Dean MP, Bub DN, Masson ME (2001) Interference from related items in object identification. J Exp Psychol Learn Mem Cogn 27: 733-743.

Debener S, Makeig S, Delorme A, Engel AK (2005) What is novel in the novelty oddball paradigm? Functional significance of the novelty P3 event-related potential as revealed by independent component analysis. Brain Res Cogn Brain Res 22: 309-321.

Debener S, Ullsperger M, Siegel M, Fiehler K, von Cramon DY, Engel AK (2005) Trial-by-trial coupling of concurrent electroencephalogram and functional magnetic resonance imaging identifies the dynamics of performance monitoring. J Neurosci 25: 11730-11737.

Deffke I, Sander T, Heidenreich J, Sommer W, Curio G, Trahms L, Lueschow A (2007) MEG/EEG sources of the 170-ms response to faces are co-localized in the fusiform gyrus. Neuroimage 35: 1495-1501.

Dell'Acqua R, Grainger J (1999) Unconscious semantic priming from pictures. Cognition 73: B1-B15.

Desimone R (1998) Visual attention mediated by biased competition in extrastriate visual cortex. Philos Trans R Soc Lond B Biol Sci 353: 1245-1255.

Desimone R, Duncan J (1995) Neural mechanisms of selective visual attention. Annu Rev Neurosci 18: 193-222.

DeYoe EA, Bandettini P, Neitz J, Miller D, Winans P (1994) Functional magnetic resonance imaging (FMRI) of the human brain. J Neurosci Methods 54: 171-187.

Di Pace E, Longoni AM, Zoccolotti P (1991) Semantic processing of unattended parafoveal words. Acta Psychol (Amst) 77: 21-34.

Dorfel D, Werner A, Schaefer M, von Kummer R, Karl A (2009) Distinct brain networks in recognition memory share a defined region in the precuneus. Eur J Neurosci 30: 1947-1959.

Dubois S, Rossion B, Schiltz C, Bodart JM, Michel C, Bruyer R, Crommelinck M (1999) Effect of familiarity on the processing of human faces. Neuroimage 9: 278-289.

Eddy M, Schmid A, Holcomb PJ (2006) Masked repetition priming and event-related brain potentials: a new approach for tracking the time-course of object perception. Psychophysiology 43: 564-568.

Eichele T, Calhoun VD, Moosmann M, Specht K, Jongsma ML, Quiroga RQ, Nordby H, Hugdahl K (2008) Unmixing concurrent EEG-fMRI with parallel independent component analysis. Int J Psychophysiol 67: 222-234.

Eimer M (2000) Event-related brain potentials distinguish processing stages involved in face perception and recognition. Clin Neurophysiol 111: 694-705.

Eimer M (2000) The face-specific N170 component reflects late stages in the structural encoding of faces. Neuroreport 11: 2319-2324.

Eimer $\mathrm{M}$, Kiss $\mathrm{M}$ The top-down control of visual selection and how it is linked to the N2pc component. Acta Psychol (Amst) 135: 100-102; discussion 133-109.

Fischl B, Sereno MI, Tootell RB, Dale AM (1999) High-resolution intersubject averaging and a coordinate system for the cortical surface. Hum Brain Mapp 8: 272-284.

Fletcher PC, Frith CD, Baker SC, Shallice T, Frackowiak RS, Dolan RJ (1995) The mind's eye--precuneus activation in memory-related imagery. Neuroimage 2: 195-200.

Forman SD, Cohen JD, Fitzgerald M, Eddy WF, Mintun MA, Noll DC (1995) Improved assessment of significant activation in functional magnetic resonance imaging (fMRI): use of a cluster-size threshold. Magn Reson Med 33: 636-647.

Fujita I, Tanaka K, Ito M, Cheng K (1992) Columns for visual features of objects in monkey inferotemporal cortex. Nature 360: 343-346. 
Furey ML, Tanskanen T, Beauchamp MS, Avikainen S, Uutela K, Hari R, Haxby JV (2006) Dissociation of face-selective cortical responses by attention. Proc Natl Acad Sci U S A 103: 1065-1070.

Galdo Alvarez S, Lindin Novo M, Diaz Fernandez F (2009) Naming faces: a multidisciplinary and integrated review. Psicothema 21: 521-527.

Ganis G, Kutas M, Sereno MI (1996) The search of 'common sense': an electrophysiological study of the comprehension of words and pictures in reading. J Cogn Neurosci 8: 89-106.

Gauthier I, Tarr MJ, Moylan J, Skudlarski P, Gore JC, Anderson AW (2000) The fusiform "face area" is part of a network that processes faces at the individual level. J Cogn Neurosci 12: 495-504.

Gentile F, Jansma BM Neural competition through visual similarity in face selection. Brain Res.

Gentile F, Jansma BM Neural competition through visual similarity in face selection. Brain Res 1351: 172 184.

Gitelman DR, Nobre AC, Parrish TB, LaBar KS, Kim YH, Meyer JR, Mesulam M (1999) A large-scale distributed network for covert spatial attention: further anatomical delineation based on stringent behavioural and cognitive controls. Brain 122 ( Pt 6): 1093-1106.

Glaser WR, Glaser MO (1989) Context effects in stroop-like word and picture processing. J Exp Psychol Gen 118: 13-42.

Goebel R, Esposito F, Formisano E (2006) Analysis of functional image analysis contest (FIAC) data with brainvoyager QX: From single-subject to cortically aligned group general linear model analysis and self-organizing group independent component analysis. Hum Brain Mapp 27: 392-401.

Goffaux V, Rossion B, Sorger B, Schiltz C, Goebel R (2009) Face inversion disrupts the perception of vertical relations between features in the right human occipito-temporal cortex. J Neuropsychol 3 : 45-67.

Grill-Spector K, Henson R, Martin A (2006) Repetition and the brain: neural models of stimulus-specific effects. Trends Cogn Sci 10: 14-23.

Grill-Spector K, Knouf N, Kanwisher N (2004) The fusiform face area subserves face perception, not generic within-category identification. Nat Neurosci 7: 555-562.

Grindrod CM, Bilenko NY, Myers EB, Blumstein SE (2008) The role of the left inferior frontal gyrus in implicit semantic competition and selection: An event-related fMRI study. Brain Res 1229: 167-178.

Grosbras MH, Laird AR, Paus T (2005) Cortical regions involved in eye movements, shifts of attention, and gaze perception. Hum Brain Mapp 25: 140-154.

Hagoort P (2005) On Broca, brain, and binding: a new framework. Trends Cogn Sci 9: 416-423.

Halgren E, Dale AM, Sereno MI, Tootell RB, Marinkovic K, Rosen BR (1999) Location of human faceselective cortex with respect to retinotopic areas. Hum Brain Mapp 7: 29-37.

Hamm JP, Johnson BW, Kirk IJ (2002) Comparison of the N300 and N400 ERPs to picture stimuli in congruent and incongruent contexts. Clin Neurophysiol 113: 1339-1350.

Hawkins HL, Hillyard SA, Luck SJ, Mouloua M, Downing CJ, Woodward DP (1990) Visual attention modulates signal detectability. J Exp Psychol Hum Percept Perform 16: 802-811.

Haxby JV, Gobbini MI, Furey ML, Ishai A, Schouten JL, Pietrini P (2001) Distributed and overlapping representations of faces and objects in ventral temporal cortex. Science 293: 2425-2430.

Haxby JV, Hoffman EA, Gobbini MI (2000) The distributed human neural system for face perception. Trends Cogn Sci 4: 223-233.

Haxby JV, Ungerleider LG, Clark VP, Schouten JL, Hoffman EA, Martin A (1999) The effect of face inversion on activity in human neural systems for face and object perception. Neuron 22: 189-199.

Herzmann G, Schweinberger SR, Sommer W, Jentzsch I (2004) What's special about personally familiar faces? A multimodal approach. Psychophysiology 41: 688-701. 
Hillyard SA, Munte TF (1984) Selective attention to color and location: an analysis with event-related brain potentials. Percept Psychophys 36: 185-198.

Hocking J, McMahon KL, de Zubicaray GI Semantic interference in object naming: an fMRI study of the postcue naming paradigm. Neuroimage 50: 796-801.

Hoffman EA, Haxby JV (2000) Distinct representations of eye gaze and identity in the distributed human neural system for face perception. Nat Neurosci 3: 80-84.

Holcomb PJ, McPherson WB (1994) Event-related brain potentials reflect semantic priming in an object decision task. Brain Cogn 24: 259-276.

Hopfinger JB, Buonocore MH, Mangun GR (2000) The neural mechanisms of top-down attentional control. Nat Neurosci 3: 284-291.

Itier RJ, Taylor MJ (2002) Inversion and contrast polarity reversal affect both encoding and recognition processes of unfamiliar faces: a repetition study using ERPs. Neuroimage 15: 353-372.

Jacques C, Rossion B (2004) Concurrent processing reveals competition between visual representations of faces. Neuroreport 15: 2417-2421.

Jacques C, Rossion B (2006) The time course of visual competition to the presentation of centrally fixated faces. J Vis 6: 154-162.

Jemel B, Pisani M, Calabria M, Crommelinck M, Bruyer R (2003) Is the N170 for faces cognitively penetrable? Evidence from repetition priming of Mooney faces of familiar and unfamiliar persons. Brain Res Cogn Brain Res 17: 431-446.

Jiang X, Rosen E, Zeffiro T, Vanmeter J, Blanz V, Riesenhuber M (2006) Evaluation of a shape-based model of human face discrimination using FMRI and behavioral techniques. Neuron 50: 159-172.

Kan IP, Thompson-Schill SL (2004) Effect of name agreement on prefrontal activity during overt and covert picture naming. Cogn Affect Behav Neurosci 4: 43-57.

Kan IP, Thompson-Schill SL (2004) Selection from perceptual and conceptual representations. Cogn Affect Behav Neurosci 4: 466-482.

Kanwisher N, McDermott J, Chun MM (1997) The fusiform face area: a module in human extrastriate cortex specialized for face perception. J Neurosci 17: 4302-4311.

Kastner S, De Weerd P, Desimone R, Ungerleider LG (1998) Mechanisms of directed attention in the human extrastriate cortex as revealed by functional MRI. Science 282: 108-111.

Kastner S, De Weerd P, Pinsk MA, Elizondo MI, Desimone R, Ungerleider LG (2001) Modulation of sensory suppression: implications for receptive field sizes in the human visual cortex. J Neurophysiol 86: 1398-1411.

Kastner S, Pinsk MA, De Weerd P, Desimone R, Ungerleider LG (1999) Increased activity in human visual cortex during directed attention in the absence of visual stimulation. Neuron 22: 751-761.

Kastner S, Ungerleider LG (2000) Mechanisms of visual attention in the human cortex. Annu Rev Neurosci 23: 315-341.

Kastner S, Ungerleider LG (2001) The neural basis of biased competition in human visual cortex. Neuropsychologia 39: 1263-1276.

Kaufmann JM, Schweinberger SR, Burton AM (2009) N250 ERP correlates of the acquisition of face representations across different images. J Cogn Neurosci 21: 625-641.

Kayser S (1985) Heads. New York: Abbeville Press.

Klem GH, Luders HO, Jasper HH, Elger C (1999) The ten-twenty electrode system of the International Federation. The International Federation of Clinical Neurophysiology. Electroencephalogr Clin Neurophysiol Suppl 52: 3-6.

Klopp J, Marinkovic K, Chauvel P, Nenov V, Halgren E (2000) Early widespread cortical distribution of coherent fusiform face selective activity. Hum Brain Mapp 11: 286-293. 
Knierim JJ, van Essen DC (1992) Neuronal responses to static texture patterns in area V1 of the alert macaque monkey. J Neurophysiol 67: 961-980.

Kreiman G, Hung CP, Kraskov A, Quiroga RQ, Poggio T, DiCarlo JJ (2006) Object selectivity of local field potentials and spikes in the macaque inferior temporal cortex. Neuron 49: 433-445.

Kremin H, Hamerel M, Dordain M, De Wilde M, Perrier D (2000) Age of acquisition and name agreement as predictors of mean response latencies in picture naming of French adults. Brain Cogn 43: 286-291.

Kutas M, Dale AM (1997) Electrical and magnetic readings of mental functions

In: Cognitive Neuroscience (Rugg MD, ed.), pp 197-237.

Kutas M, Hillyard SA (1980) Reading senseless sentences: brain potentials reflect semantic incongruity. Science 207: 203-205.

Lange JJ, Wijers AA, Mulder LJ, Mulder G (1998) Color selection and location selection in ERPs: differences, similarities and 'neural specificity'. Biol Psychol 48: 153-182.

Law I, Svarer C, Holm S, Paulson OB (1997) The activation pattern in normal humans during suppression, imagination and performance of saccadic eye movements. Acta Physiol Scand 161: 419-434.

Lawrence NS, Ross TJ, Hoffmann R, Garavan H, Stein EA (2003) Multiple neuronal networks mediate sustained attention. J Cogn Neurosci 15: 1028-1038.

Levelt WJ, Praamstra P, Meyer AS, Helenius P, Salmelin R (1998) An MEG study of picture naming. J Cogn Neurosci 10: 553-567

Levelt WJM, Schriefers H, Vorberg D, Meyer AS, Pechmann T, \& Havinga J (1991) The time course of lexical access in speech production: A study of picture naming. Psychological Review 98: 122-142.

Loffler G, Yourganov G, Wilkinson F, Wilson HR (2005) fMRI evidence for the neural representation of faces. Nat Neurosci 8: 1386-1390.

Luck SJ, Chelazzi L, Hillyard SA, Desimone R (1997) Neural mechanisms of spatial selective attention in areas V1, V2, and V4 of macaque visual cortex. J Neurophysiol 77: 24-42.

Luck SJ, Hillyard SA (1994) Electrophysiological correlates of feature analysis during visual search. Psychophysiology 31: 291-308.

Luck SJ, Hillyard SA, Mouloua M, Woldorff MG, Clark VP, Hawkins HL (1994) Effects of spatial cuing on luminance detectability: psychophysical and electrophysiological evidence for early selection. J Exp Psychol Hum Percept Perform 20: 887-904.

Makeig S, Debener S, Onton J, Delorme A (2004) Mining event-related brain dynamics. Trends Cogn Sci 8: 204-210.

Makeig S, Westerfield M, Jung TP, Covington J, Townsend J, Sejnowski TJ, Courchesne E (1999) Functionally independent components of the late positive event-related potential during visual spatial attention. J Neurosci 19: 2665-2680.

Makeig S, Westerfield M, Jung TP, Enghoff S, Townsend J, Courchesne E, Sejnowski TJ (2002) Dynamic brain sources of visual evoked responses. Science 295: 690-694.

Makeig S, Westerfield M, Townsend J, Jung TP, Courchesne E, Sejnowski TJ (1999) Functionally independent components of early event-related potentials in a visual spatial attention task. Philos Trans R Soc Lond B Biol Sci 354: 1135-1144.

Martin-Loeches M (2007) The gate for reading: reflections on the recognition potential. Brain Res Rev 53: 89-97.

Martin-Loeches M, Hinojosa JA, Gomez-Jarabo G, Rubia FJ (2001) An early electrophysiological sign of semantic processing in basal extrastriate areas. Psychophysiology 38: 114-124.

Martin A, Chao LL (2001) Semantic memory and the brain: structure and processes. Curr Opin Neurobiol 11: 194-201. 
McPherson WB, Holcomb PJ (1999) An electrophysiological investigation of semantic priming with pictures of real objects. Psychophysiology 36: 53-65.

Milham MP, Banich MT, Webb A, Barad V, Cohen NJ, Wszalek T, Kramer AF (2001) The relative involvement of anterior cingulate and prefrontal cortex in attentional control depends on nature of conflict. Brain Res Cogn Brain Res 12: 467-473.

Miller EK, Gochin PM, Gross CG (1993) Suppression of visual responses of neurons in inferior temporal cortex of the awake macaque by addition of a second stimulus. Brain Res 616: 25-29.

Moran J, Desimone R (1985) Selective attention gates visual processing in the extrastriate cortex. Science 229: 782-784.

Nasanen R (1999) Spatial frequency bandwidth used in the recognition of facial images. Vision Res 39: 3824-3833.

Nasr S, Esteky H (2009) A study of N250 event-related brain potential during face and non-face detection tasks. J Vis 9: 5 1-14.

Nobre AC, Sebestyen GN, Gitelman DR, Mesulam MM, Frackowiak RS, Frith CD (1997) Functional localization of the system for visuospatial attention using positron emission tomography. Brain 120 ( Pt 3): 515-533.

Okita T, Wijers AA, Mulder G, Mulder LJ (1985) Memory search and visual spatial attention: an eventrelated brain potential analysis. Acta Psychol (Amst) 60: 263-292.

Onton J, Delorme A, Makeig S (2005) Frontal midline EEG dynamics during working memory. Neuroimage 27: 341-356.

Onton J, Makeig S (2006) Information-based modeling of event-related brain dynamics. Prog Brain Res 159: 99-120.

Op de Beeck HP, Dicarlo JJ, Goense JB, Grill-Spector K, Papanastassiou A, Tanifuji M, Tsao DY (2008) Finescale spatial organization of face and object selectivity in the temporal lobe: do functional magnetic resonance imaging, optical imaging, and electrophysiology agree? J Neurosci 28: 11796-11801.

Oppenheim GM, Dell GS, Schwartz MF The dark side of incremental learning: a model of cumulative semantic interference during lexical access in speech production. Cognition 114: 227-252.

Pessoa L, Kastner S, Ungerleider LG (2003) Neuroimaging studies of attention: from modulation of sensory processing to top-down control. J Neurosci 23: 3990-3998.

Petit L, Dubois S, Tzourio N, Dejardin S, Crivello F, Michel C, Etard O, Denise P, Roucoux A, Mazoyer B (1999) PET study of the human foveal fixation system. Hum Brain Mapp 8: 28-43.

Pfutze EM, Sommer W, Schweinberger SR (2002) Age-related slowing in face and name recognition: evidence from event-related brain potentials. Psychol Aging 17: 140-160.

Posner MI, Snyder CR, Davidson BJ (1980) Attention and the detection of signals. J Exp Psychol 109: 160174.

Price CJ The anatomy of language: a review of $100 \mathrm{fMRI}$ studies published in 2009. Ann N Y Acad Sci 1191: $62-88$.

Recanzone GH (2000) Spatial processing in the auditory cortex of the macaque monkey. Proc Natl Acad Sci U S A 97: 11829-11835.

Reddy L, Kanwisher NG, VanRullen R (2009) Attention and biased competition in multi-voxel object representations. Proc Natl Acad Sci U S A 106: 21447-21452.

Reinholz J, Pollmann S (2007) Neural basis of redundancy effects in visual object categorization. Neurosci Lett 412: 123-128.

Reynolds JH, Chelazzi L, Desimone R (1999) Competitive mechanisms subserve attention in macaque areas V2 and V4. J Neurosci 19: 1736-1753. 
Reynolds JH, Desimone R (1999) The role of neural mechanisms of attention in solving the binding problem. Neuron 24: 19-29, 111-125.

Reynolds JH, Pasternak T, Desimone R (2000) Attention increases sensitivity of V4 neurons. Neuron 26: 703-714.

Riecke L, Esposito F, Bonte M, Formisano E (2009) Hearing illusory sounds in noise: the timing of sensory-perceptual transformations in auditory cortex. Neuron 64: 550-561.

Rizzolatti G, Riggio L, Dascola I, Umilta C (1987) Reorienting attention across the horizontal and vertical meridians: evidence in favor of a premotor theory of attention. Neuropsychologia 25: 31-40.

Rossion B, Caldara R, Seghier M, Schuller AM, Lazeyras F, Mayer E (2003) A network of occipito-temporal face-sensitive areas besides the right middle fusiform gyrus is necessary for normal face processing. Brain 126: 2381-2395.

Rossion B, Delvenne JF, Debatisse D, Goffaux V, Bruyer R, Crommelinck M, Guerit JM (1999) Spatiotemporal localization of the face inversion effect: an event-related potentials study. Biol Psychol 50: 173-189.

Sams M, Alho K, Naatanen R (1983) Sequential effects on the ERP in discriminating two stimuli. Biol Psychol 17: 41-58.

Sato T, Uchida G, Tanifuji M (2008) Cortical Columnar Organization Is Reconsidered in Inferior Temporal Cortex. Cereb Cortex.

Schendan HE, Kutas M (2002) Neurophysiological evidence for two processing times for visual object identification. Neuropsychologia 40: 931-945.

Schweinberger SR, Huddy V, Burton AM (2004) N250r: a face-selective brain response to stimulus repetitions. Neuroreport 15: 1501-1505.

Schweinberger SR, Kaufmann JM, Moratti S, Keil A, Burton AM (2007) Brain responses to repetitions of human and animal faces, inverted faces, and objects: an MEG study. Brain Res 1184: 226-233.

Schweinberger SR, Pickering EC, Jentzsch I, Burton AM, Kaufmann JM (2002) Event-related brain potential evidence for a response of inferior temporal cortex to familiar face repetitions. Brain Res Cogn Brain Res 14: 398-409.

Sergent J, Ohta S, MacDonald B (1992) Functional neuroanatomy of face and object processing. A positron emission tomography study. Brain 115 Pt 1: 15-36.

Sitnikova T, West WC, Kuperberg GR, Holcomb PJ (2006) The neural organization of semantic memory: Electrophysiological activity suggests feature-based segregation. Biol Psychol 71: 326-340.

Snijders TM, Vosse T, Kempen G, Van Berkum JJ, Petersson KM, Hagoort P (2009) Retrieval and unification of syntactic structure in sentence comprehension: an FMRI study using word-category ambiguity. Cereb Cortex 19: 1493-1503.

Steeves JK, Culham JC, Duchaine BC, Pratesi CC, Valyear KF, Schindler I, Humphrey GK, Milner AD, Goodale MA (2006) The fusiform face area is not sufficient for face recognition: evidence from a patient with dense prosopagnosia and no occipital face area. Neuropsychologia 44: 594-609.

Steyvers M (2002) Multidimensional Scaling. In: Encyclopedia of Cognitive Science. London, UK: Nature Publishing Group.

Steyvers M, \& Busey, T. (2000) Predicting Similarity Ratings to Faces using Physical Descriptions. In: Computational, geometric, and process perspectives on facial cognition: Contexts and challenges: Lawrence Erlbaum Associates.

Tamura H, Kaneko H, Fujita I (2005) Quantitative analysis of functional clustering of neurons in the macaque inferior temporal cortex. Neurosci Res 52: 311-322. 
Tanaka JW, Curran T, Porterfield AL, Collins D (2006) Activation of preexisting and acquired face representations: the $\mathrm{N} 250$ event-related potential as an index of face familiarity. J Cogn Neurosci 18: 1488-1497.

Tanaka K (2003) Columns for complex visual object features in the inferotemporal cortex: clustering of cells with similar but slightly different stimulus selectivities. Cereb Cortex 13: 90-99.

Theeuwes J Top-down and bottom-up control of visual selection. Acta Psychol (Amst) 135: 77-99.

Thompson-Schill SL, D'Esposito M, Aguirre GK, Farah MJ (1997) Role of left inferior prefrontal cortex in retrieval of semantic knowledge: a reevaluation. Proc Natl Acad Sci U S A 94: 14792-14797.

Thomsen T, Specht K, Ersland L, Hugdahl K (2005) Processing of conflicting cues in an attention-shift paradigm studied with fMRI. Neurosci Lett 380: 138-142.

Tipper SP, Driver J (1988) Negative priming between pictures and words in a selective attention task: evidence for semantic processing of ignored stimuli. Mem Cognit 16: 64-70.

Tipper SP, MacQueen GM, Brehaut JC (1988) Negative priming between response modalities: evidence for the central locus of inhibition in selective attention. Percept Psychophys 43: 45-52.

Treisman AM (1969) Strategies and models of selective attention. Psychol Rev 76: 282-299.

Treue S, Martinez Trujillo JC (1999) Feature-based attention influences motion processing gain in macaque visual cortex. Nature 399: 575-579.

Treue S, Maunsell JH (1996) Attentional modulation of visual motion processing in cortical areas MT and MST. Nature 382: 539-541.

Vigliocco G, Vinson DP, Damian MF, Levelt W (2002) Semantic distance effects on object and action naming. Cognition 85: B61-69.

Wang G, Tanifuji M, Tanaka K (1998) Functional architecture in monkey inferotemporal cortex revealed by in vivo optical imaging. Neurosci Res 32: 33-46.

West WC, Holcomb PJ (2002) Event-related potentials during discourse-level semantic integration of complex pictures. Brain Res Cogn Brain Res 13: 363-375.

Wheatley T, Weisberg J, Beauchamp MS, Martin A (2005) Automatic priming of semantically related words reduces activity in the fusiform gyrus. J Cogn Neurosci 17: 1871-1885.

Wijers AA, Mulder G, Okita T, Mulder LJ, Scheffers MK (1989) Attention to color: an analysis of selection, controlled search, and motor activation, using event-related potentials. Psychophysiology 26: 89109.

Wijers AA, Otten LJ, Feenstra S, Mulder G, Mulder LJ (1989) Brain potentials during selective attention, memory search, and mental rotation. Psychophysiology 26: 452-467.

Williams EJ (1949) Experimental designs balanced for the estimation of residual effects of treatments. Australian Journal of Scientific Research 2 149-168.

Wojciulik E, Kanwisher N, Driver J (1998) Covert visual attention modulates face-specific activity in the human fusiform gyrus: fMRI study. J Neurophysiol 79: 1574-1578.

Yee PL (1991) Semantic inhibition of ignored words during a figure classification task. Q J Exp Psychol A 43: $127-153$

Zhang Y, Guo C (2008) Relationship between perceptual and semantic levels of representation: An event-related potential study. Chinese Science Bulletin 53: 3847-3859. 
Summary 
The aim of the present work was to investigate the underlying neural mechanism of face selection in the presence of a distracting face. We explored this particular topic via two different perspectives. In fact, the relevance of a face in the pair was in one case related to the visual properties of that face (Chapter 2 and Chapter 3 ) and in the other studies (Chapter 4 and Chapter 5 ) to its semantic information. The combination of these two lines of research allowed a better understanding of the mechanism of face selection and the integrative process regarding the physical and semantic information that are associated with a target face. In Chapter 1 we outlined the rationale of the thesis and the technique and procedures used to test our hypothesis. In particular, we investigated whether the selection mechanism in the semantic as well as in the visual domain, worked according to the competitive models of selection. According to these models, when two items (e.g. faces) are simultaneously presented in the visual field and none of them is attended to (or selected), their perceptual or semantic information compete to be represented at the neural level. When one of the two items becomes relevant for behavior, selection occurs by shifting the focus of attention to the target. This biases competitive interactions towards the processing of that item only. The competition hypothesis was tested by simultaneously presenting two faces and by asking the participants to ignore both of them (ignore-faces task). The bias account was explored with an attend-to-face task where the subject had to covertly attend to one of the two faces. We used functional magnetic resonance imaging ( $f M R I)$ to identify the brain regions involved in face selection and to find out whether such a mechanism worked in a competitive fashion. The electroencephalography (EEG) technique was used in order to track the temporal dynamics of the two stages of selection that are hypothesized by competitive models: competition and bias effect. In Chapter 2 we described in more detail the fMRI experiment that was used to investigate the visual selection of a face in the context of a distractor. In this study the two faces presented were either similar or dissimilar to each other. We found that the two face selective areas, the fusiform face area (FFA) and the occipital face area (OFA) were modulated by the similarity manipulation only within the ignore-faces condition and not in the attend-to-face condition. The attend-to-face task consisted, in this particular study, of a match-to-face task where participants performed a button-press when the face on the left matched the one presented in the instruction phase. These results were interpreted in line with the competitive models of selection. First, the similarity effect that was found only in the ignore-faces condition suggested that both faces were processed to some extent. Moreover, the lower fMRI signal in the similar compared to the dissimilar condition, reflected the occurrence of competitive interactions between the two faces. In fact, according to the neural representation of the FFA, similar faces are processed by neighboring and overlapping neural populations. This would lead consequently to a stronger competition when similar faces would be presented compared to two dissimilar faces. The lack of modulation in the attend-to-face condition suggested that attention inhibited the influence of the distractor and biased competition towards the visual processing of the target. The same stimuli, tasks and design, as described in Chapter 2, were used in an EEG experiment that we illustrated in 
Chapter 3. The EEG signal observed at the occipito-temporal location in a 250 time-window reflected the same pattern of activation that we found in OFA and FFA. More specifically, we observed at the level of the N250 ERP component that in the ignore-faces condition similar faces elicited a lower EEG signal compared to dissimilar face. In addition, this modulation was cancelled out by attending to one of the two faces (attend-to-face condition). In sum, the EEG experiment suggested that both the competitive and the bias phases of selection occurred in a late stage of face processing. In Chapter 4, we reported the fMRI experiment on the semantic selection of a face in the presence of another face. Similarly to the visual selection experiments we expected that this mechanism might work according to the competitive model of selection. Therefore, in analogy to the design above mentioned, we tested the competition and bias hypothesis by presenting two faces that had to be respectively ignored or (one of them) attended to. Moreover, as the selection occurred with regard of the semantic information of the target face each face was associated with a specific profession. Professions, associated with the pair of faces, could belong to the same (related faces) or to a different (unrelated faces) category. We observed in the inferior frontal gyrus (IFG) a relatedness effect in the ignore-faces condition and no modulation in the attend-to-face condition. In line with the interpretation of previous results in OFA and FFA (see fMRI results in Chapter 2) and in the N250 time-window (see EEG results in Chapter 3), we suggested that when the two faces were unattended to their semantic information was automatically processed and competed for representation. Attention resolved competition by favoring the processing of the profession information of the target face. In this experiment we also found that FFA was modulated by relatedness in the attend-to-face condition. The direction of this effect (attend-to-related-face > attend-to-unrelated-face) indicated that at this stage of face processing attention was used to integrate the semantic and perceptual information of the target face in FFA. The temporal profile of these neural events was outlined in the EEG experiment, described in Chapter 5. We observed that the integrative process in FFA (see fMRI results in Chapter 4) occurred, most likely, in a 250 time-window, as we found the same relatedness modulation within the attend-to-face condition at the level of the N250 in occipital-temporal sites. Finally, we observed in a frontal N300 a relatedness effect in the ignore-faces condition similar to the one we found in the IFG (see fMRI results in Chapter 4). However, unlike the pattern of activation of the IFG the N300 did show a relatedness modulation also in the attend-to-face condition. A similar lack of relatedness effect we observed in the IFG was found at the level of the parietal N400. These results suggested that the competitive and bias phases of selection took place respectively in the $300 \mathrm{~ms}$ and $400 \mathrm{~ms}$ timewindows. Finally, in Chapter 6, we draw the general conclusions from the four experiments. In summary, when a face has to be selected in the presence of another face on the basis of visual properties, this selection mechanism occurs in FFA and OFA in a late stage of face processing $(250 \mathrm{~ms})$ and according to the competitive models of selection (Chapter 2 and Chapter 3). Moreover, in Chapter 4 and Chapter 5 we observed that the same models hold if the relevance of the face to be selected is changed from visual to semantic information. We 
also found a spatial and temporal shift in the regions involved in such a mechanism. Especially, selection occurred in the IFG between 300 and 400 ms. FFA, in this case, was involved in a earlier stage of selection when the integration between the semantic and facial feature of the target face took place. 


\section{Samenvatting}

Het doel van de huidige studie was om het neurale mechanisme te onderzoeken dat gezichten selecteert in de aanwezigheid van afleidende gezichten. We onderzochten dit onderwerp vanuit twee verschillende perspectieven. De relevantie van een gezicht was danwel gerelateerd aan de visuele eigenschappen van dat gezicht (hoofdstuk 2 en 3), danwel gerelateerd aan semantische informatie ervan (hoofdstuk 4 en 5). Dankzij de combinatie van deze twee onderzoekslijnen konden we beter begrijpen hoe het mechanisme dat gezichten selecteert werkt, en hoe fysieke en semantische informatie van het betreffende gezicht worden geïntegreerd. In hoofdstuk 1 beschreven we de gedachte achter deze thesis, alsmede de technieken en de procedures die we gebruikten om onze hypothese te testen. Met name hebben we onderzocht of het selectiemechanisme, zowel in het visuele als in het semantische domein, werkten aan de hand van competitieve selectiemodellen. Volgens deze modellen ontstaat een competitie om neurale representatie tussen de perceptuele en semantische representaties van twee items (b.v. gezichten), wanneer deze tegelijkertijd in het visuele veld gepresenteerd worden en wanneer aan beiden geen aandacht wordt besteed. Wanneer één van beide items relevant wordt voor een bepaald gedrag, wordt het item geselecteerd door het verschuiven van de aandacht naar het relevante item. Hierdoor worden competitieve interacties bevoordeeld om enkel dat item te verwerken. De competitiehypothese werd getest door tegelijkertijd twee gezichten te presenteren en door de proefpersonen te vragen om beide te negeren ('ignore-faces' taak). De biashypothese werd getest door middel van een 'attend-to-face' taak. In deze taak werd de proefpersoon gevraagd om zijn/haar aandacht te richten op één van de gezichten. Aan de hand van functional magnetic resonance imaging (fMRI) probeerden we de hersengebieden te identificeren die betrokken zijn bij gezichtsselectie en te onderzoeken of deze selectie plaatsvond met behulp van competitieve processen. Daarnaast werd electroencephalography (EEG) gebruikt om de temporele dynamiek te achterhalen van de twee fases van selectie die genoemd worden in de competitiemodellen (het competitie- en het biaseffect). In hoofdstuk 2 beschreven we het fMRI experiment dat gebruikt werd om de visuele selectie van een gezicht, in aanwezigheid van een aandachtsafleidend gezicht, te onderzoeken. In deze studie waren deze twee gezichten danwel gelijk, danwel verschillend aan elkaar (gelijkenismanipulatie). De 'attend-to-face' conditie bestond uit een 'match-to-face' taak, waarin de proefpersoon gevraagd werd om op 
een knop te drukken als het gezicht gepresenteerd aan de linkerzijde overeenkwam met het gezicht dat tijdens de instructiefase getoond werd. We ontdekten dat de twee gezichtsselectiegebieden fusiform face area (FFA) en occipital face area (OFA) enkel in de 'ignore faces' conditie en niet in de 'attend-to-face' conditie gemoduleerd werden door de gelijkenismanipulatie. In overeenstemming met de competitieve modellen, suggereerde het effect van gelijkenis (dat enkel in de 'ignore-faces' conditie werd gevonden) dat beide gezichten in zekere mate verwerkt werden. Daarnaast weerspiegelde het lage fMRI signaal in de gelijkende conditie, vergeleken met de ongelijkende conditie, het bestaan van competitieve interacties tussen de twee gezichten. Volgens de neurale representatie van FFA, worden op elkaar gelijkende gezichten verwerkt door naburige en overlappende neuronenpopulaties. Wanneer twee gelijkende gezichten samen gepresenteerd worden, zou dit doorgaans tot een sterkere competitie leiden dan wanneer deze twee gezichten verschillend zijn. Het ontbreken van modulatie in de 'attend-to-face' conditie suggereerde dat aandacht de invloed van de aandachtsafleider onderdrukte en de competitie bevoordeelde voor de visuele verwerking van het relevante gezicht. Dezelfde stimuli, taken en design die werden beschreven in hoofdstuk 2 werden gebruikt in een EEG experiment dat beschreven werd in hoofdstuk 3. Het EEG signaal in deze studie vertoonde hetzelfde activatiepatroon dat we vonden in OFA en FFA. Daarnaast observeerden we competitie en een bias voor aandacht, $250 \mathrm{~ms}$ na stimuluspresentatie, op een occipitale-temporale locatie. Daarbij observeerden we dat gelijkende gezichten in de 'ignore-faces' conditie rond de N250 ERP component een lager EEG signaal induceren vergeleken met gezichten die verschilden. Daar komt bij dat deze modulatie teniet werd gedaan wanneer er aandacht geschonken werd aan één van de twee gezichten ('attend-to-face' conditie). We kunnen hieruit concluderen dat zowel de competitie- als de biasfase pas in een later stadium in het gezichtsverwerkingsproces plaatsvinden. In hoofdstuk 4 beschreven we het fMRI experiment, waarin de semantische selectie van een gezicht, in aanwezigheid van een ander gezicht, werd onderzocht. Zoals in de visuele selectie-experimenten verwachtten we dat dit mechanisme zou kunnen handelen volgens het competitieve selectiemodel. Daarom, in overeenstemming met het design dat hierboven beschreven werd, testten we de competitie- en biashypothese door twee gezichten te presenteren, die danwel genegeerd moesten worden, danwel moest er aan één van beiden aandacht geschonken worden. Daarnaast werd elk gezicht gekoppeld aan een specifiek beroep, aangezien de selectie plaatsvond aan de hand van semantische informatie geassocieerd met de gezichten. De beroepen, die met de gezichtsparen geassocieerd werden, konden tot eenzelfde (gerelateerde gezichten) of tot een verschillende (ongerelateerde gezichten) beroepscategorie behoren. We vonden een gerelateerdheidseffect in de inferior frontal gyrus (IFG) in de 'ignore-faces' conditie, en geen modulatie in de 'attend-to-face' conditie. Gelet op de interpretaties van de vorige resultaten in OFA en FFA (zie fMRI resultaten) en in de ERP rond 250ms na stimuluspresentatie (zie EEG resultaten), interpreteerden we deze semantische resultaten als volgt. Wanneer beide gezichten genegeerd werden, werd hun semantische informatie automatisch verwerkt en deze informatie ging vervolgens competi- 
tie aan voor representatie. Aandacht loste deze competitie op door de verwerking van het beroep van het relevante gezicht te bevoordelen. In dit experiment ontdekten we ook dat FFA gemoduleerd werd door het effect van gelijkenis in de 'attend-to-face' conditie. De richting van dit effect ('attend-to-related-faces'> 'attend-to-unrelated-faces') toonde aan dat in dit stadium van gezichtsverwerking, aandacht gebruikt wordt om semantische en perceptuele informatie van het relevante gezicht te integreren in FFA. Het temporele profiel van deze neurale gebeurtenissen werd beschreven in het EEG experiment in hoofdstuk 5 . We ontdekten dat het proces van de integratie van het percept en de semantiek dat we zagen in FFA (zie fMRI resultaten), waarschijnlijk $250 \mathrm{~ms}$ na stimuluspresentatie plaatsvond, aangezien we dezelfde gelijkenismodulatie vonden in de 'attend-to-face' conditie ter hoogte van de N250 aan occipitale-temporale gebieden. Ten slotte vonden we een effect van gelijkenis in de 'ignore-faces' conditie in een frontale N300, een effect dat overeenkomt met het effect gevonden in de IFG (zie fMRI resultaten). Echter, in tegenstelling tot het activatiepatroon van IFG, toonde de N300 een gelijkenismodulatie in de 'attend-to-faces' conditie. Ook in de pariëtale N400 vonden we een effect van gelijkenis, terwijl dit effect ontbrak in de IFG. Deze resultaten suggereerden dat de competitie- en biasfasen van selectie respectievelijk $300 \mathrm{~ms}$ en $400 \mathrm{~ms}$ na stimuluspresentatie plaatsvonden. Ten slotte trokken we in hoofdstuk 6 algemene conclusies aan de hand van de vier experimenten.

Samenvattend kunnen we stellen dat wanneer een gezicht in de aanwezigheid van een ander gezicht geselecteerd moet worden op basis van visuele eigenschappen, dit selectiemechanisme plaatsvindt in FFA en OFA in een laat stadium van gezichtsverwerking (250ms) en werkt volgens het competitieve selectiemodel (hoofdstuk 2 en 3). Daarnaast vonden we in hoofdstuk 4 en 5 dat hetzelfde model van kracht is wanneer de relevantie van het te selecteren gezicht verschuift van visuele naar semantische informatie. Verder observeerden we een spatiële en temporele verschuiving in de gebieden die betrokken zijn bij een dergelijk mechanisme. We zagen namelijk dat selectie in de IFG tussen 300 en $400 \mathrm{~ms}$ plaatsvond. In dit geval, wanneer de integratie van het semantische- en het gezichtskenmerk van het relevante gezicht plaats moest vinden, was FFA betrokken bij een eerder selectiestadium. 



\section{Acknowledgments}

Finally, there are many people that I need to thank for their support in such a long pathway towards the "ultimate wisdom". However, I won't list those persons according to their importance because for me they were all equally important in their own (small or big) ways. I will proceed chronologically and for that I will trust on my long term memory.

Non ho bisogno di un grande sforzo per ricordare l'espressione di quei tre visi appiccicati l'uno con l'altro sul ciglio di quella porta al sesto piano di via America Latina 68 più di sette anni fa. Don Raffaele, Donna Assunta e la piccola Stefania erano lì con i loro occhioni da cerbiatto per augurarmi buon viaggio e soprattutto per le ultime raccomandazioni: "mi raccomando, mangia, figlio mio", "non prendere freddo, lassù al polo, gela tutto l'anno" "chiamaci ogni giorno, nun te scurda' da famiglia". Non rimaneva che tagliare quel cordone ombelicale che ci aveva sempre tenuto stretti incondizionatamente. Li ringrazio per non averlo fatto. Li ringrazio per aver usato quel filo magico per seguirmi ovunque andassi. Mi ha permesso di vedere cosa succedesse lì fuori attraverso le trasparenze e il calore di una piccola finestra al sesto piano.

....and the adventure started. After 1550 kilometers up north, a Neapolitan accent made me feel as I actually never moved. Dear Elia and Milene, you soon welcomed me as someone of your family. When I came for dinner to your place, the great scent coming from your kitchen made me fly 'ncoppa o' Vesuvio and swim in the Mediterranean sea. Thank you guys for all that.

Thanks again, Elia, because you introduced me to the colorful and mysterious world of research. Your way of working fascinated me since the beginning. The freedom you gave me to search for solutions to problems I never heard about was something completely new for me, but absolutely exciting. If there is someone to thank (or blame) for me to have started such an adventurous career, it is definitely you. Thanks and Forza Roma.

At the time of my arrival in Maastricht another Italian was there. You, Federico, already knew a lot of things about this charming city and worldwide recognized lab. Thanks to you, after a few days I also knew almost everything I needed to know. You taught me "la pasta alla parmigiana con un botto d'olio", you made me win all the time when we played tennis and finally, it is with you that I organized the first house-party of my staying in Maastricht (the first out of many). 
As is true for the $99 \%$ of the exchange students, the first place I lived in was the guesthouse in Brouwersweg 100. Although, my room was 4 square meters and the building was not exactly like Buckingham Palace, Magnus, Simon, Doris, Sophie and all the others soon made me feel like home. Thanks for the crazy dances at the Twee Heren, for all the beers at the Highlander, for all the international dinners, for Halloween and the famous Puma Night (for that night, I also need to thank the police of Maastricht, by the way). I will never forget all those insane experiences we shared together. Thank you all.

Dear Alessia, now it's your turn (chronologically speaking). I won't describe here how a great friend you were and are. It won't fit with my character (with you). However, you deserve a special thank (and also you Teresa) for the new approach you used to teach me on how to improve my English. The technique was pretty much straightforward: making fun of me every time I tried to convey a difficult concept ("the pen is on the table"). A mystery still remains unsolved: why none of the jokes I made on you, worked out?

While the gang in Maastricht was growing up, every couple of months, I was looking forward to rejoin those people that since my childhood I share the most amazing experiences with: gli amici miei. Vi ringrazio tutti, per le partite di calcetto di via Farsalo, per le mille maschere che vi ho chiesto di indossare, e per quelle che vi siete tolti, per tutte le feste diverse, e per quelle uguali, per i viaggi della vita, e per quelli che mi hanno fatto annusare il profumo della morte. Insomma malgrado la distanza, malgrado le enormi differenze intellettuali e socio-economiche e malgrado tutte le a..lità, vi ringrazio per essere rimasti i miei migliori amici.

After writing my master thesis, one day in the cold winter of 2006, I was proposed to stay four years longer and my PhD project started. Together with the official position I also got a desk and a computer, an office and two extraordinary office-mates, Joel and Vincent. I don't know, guys, whether I should start with the "Excuses" or with the "Thanks". Let's start with a big SORRY for all the singing, the drumming, the whistling and the footing (i.e. annoyingly and rhythmically beating the foot on the ground). In summary, sorry for all the musical sounds I produced everyday. I always justified myself by saying that it was because of my loud "italianess" or because it helped me to concentrate. Sorry for all those lies as well: I just like music. Thanks for the infinite amount of topics I had the chance to discuss with you. Digging into the italian history to finally understand why Italians still vote Berlusconi, talking for three days in a row about the "Antichrist", trying to find out the supreme reason of the continuation of the human specie, traveling together within the mysterious world of the basal ganglia, all that was extremely enriching, pleasant and very fun. Thanks for building up together the most fine and humbly intellectual movie club of the whole university. In a few words: thanks to have been my guardian angels.

Bernie, you have been an exquisite supervisor and, above all, a life guide. It was an honor to work with you for such a long time. You taught me almost everything I know about neuroscience, research and team-working. Your endless knowledge in several fields and about the most various topics gave me the opportunity to look at my work from several 
perspectives in order to be constructively critic while building up a design or interpreting an experimental finding. And last but not least, I have to thank you to have seasoned this learning process with a delightful atmosphere consisting of enthusiasm, friendship and a lot of fun.

In the first period of my $\mathrm{PhD}, \mathrm{I}$ had the chance to meet someone who made the passage from being a cold engineer to a quasi-neuroscientist incredibly smooth. My Caronte is called Mart. Thanks of you I learned the art of designing an experimental design. Discussing about my first findings with you was a real pleasure. You taught me that in order to be good scientists we need to be very strict reviewers of ourselves. You taught me all that and much more. Thanks.

The huge hole left by Mart was filled up few years later by another very sMart guy. Dear Job, thanks for the volcanic enthusiasm and freshly great ideas you soon brought into the group.

None of the data I have acquired, analyzed and commented would have existed without the active presence of all the subjects that participated in my experiments. You provided me with the best brains "on the market". Thank you very much for your patience, your "covert attentional" skills and excellent face processing.

A special thank goes to Els, Pim, Sebastian and Isabelle, who helped me measuring and analyzing the EEG data. Without you, guys, my thesis would have been much shorter.

Experiments, scientific discussions, papers and teaching are very important for a $\mathrm{PhD}$ student. However, a fruitful working day has to be accompanied by several non-workingmoments. In all these years I was particularly lucky to have spent those moments with three special persons: Nick, Martin and Giancarlo. Thanks to you guys, every lunch or coffeetje was a surrealistic trip through unexplored topics, non-senses and black jokes. But the fact that you are the funniest colleagues is not the only reason you are included in the last pages of my thesis. Above all, I need to thank you because you demonstrated to belong to a special category of people that I call friends, real friends.

Seven years is a very long period and an Italian cannot stay so many years without constantly training his accent, singing Italian traditional songs and sharing Italian recipes with other Italians (at least this is what $99 \%$ of the world population thinks...and I like to keep cliché alive). Fortunately, I found the best Italian community I could ever imagine. Cari Cabrio, Charetta e Roscio, voi siete stati tra i primi a farmi sentire come a casa. Grazie per avermi assecondato come si fa con i pazzi. Grazie per esservi rotti la spalla per me in modo figurato e non. Grazie per avermi sostenuto quando stavo per cadere. Grazie per aver vinto un mondiale come pochi altri e per averlo rivinto l'anno dopo anche di più. Grazie per essere stati un riferimento e anche di più. And thanks Willem to have created the perfect place and the perfect atmosphere to experience all that. Ma "the little Italy" from Maastricht non finisce qui. Mancano all'appello sei incredibili personaggi: Giacomo Stefano, Arianna, AmicoMi', Cinzia e Craig (who, although he is not Italian, has all the cooking skills of a real Italian mother). Grazie per tutte le cenette da tre stelle michelin (a casa mia) e per quelle un po' più 
umili che normalmente facevamo da voi. Grazie per le interminabili ore passate all'AH per poi decidere di mangiare pasta "aglio e oglio". Grazie per essere stati dei buoni allievi quando v'insegnavo a giocare a calcetto. Grazie per tutti i poker "dinamici", per essere dei decisionisti e soprattutto to the point. Grazie per tutta la follia che mi avete generosamente regalato e per quella continueremo a condividere.

I'm almost at the end. Only one person is still needed to be thanked. As I said at the beginning, the list of people in this "small" acknowledgement part was ordered chronologically. For this person I did not manage to find a place in time. Dear Pat, you have been always there also when you were not and even before I was aware of your existence. Your freedom and intelligence has been a source of inspiration for my work and my intellectual growth. Thanks to you I understood the importance of integrity and the value of generosity. Thanks for your cooking skills, your funny (but perfect) Italian and your cute humor. Your jokes and your patience have been fundamental all the moments I was a tiny very little stressed while writing my PhD thesis. Thanks to be different. Thanks to have made a "me and you" a loving "us". 


\section{Curriculum Vitae}

Francesco Gentile was born on September 14th in Rome, Italy. In 1997 he completed his secondary school education at the "Liceo Scentifico, F. Severi" in Frosinone. In the same year, he was enrolled at the Faculty of Engineering at the Sapienza University in Rome. In October 2003, he did a 6-month internship on the "Independent component analysis (ICA) of functional magnetic resonance imaging (fMRI) data" at the department of Cognitive Neuroscience in the Faculty of Psychology and Neuroscience at Maastricht University. He obtained his master degree in electronic engineering in April 2004. Funded by a grant from the Sapienza University, Francesco spent one year working on "The active cancellation of the fMRI scanner noise" together with his supervisor, Elia Formisano. He started a Ph.D project in January 2006 under the supervision of Prof. Dr Bernadette Jansma at the department of Cognitive Neuroscience in the Faculty of Psychology and Neuroscience at Maastricht University. 